\title{
Analysis of Mdm38 function in mitochondrial translation
}

\author{
Dissertation
}

for the award of the degree

"Doctor rerum naturalium"

of the Georg-August-Universität Göttingen

\author{
submitted by \\ Jan-Moritz Wuttke \\ from Peine
}

Göttingen, 2015 
Member of the Thesis Committee (Reviewer):

Prof. Dr. Peter Rehling

Department of Cellular Biochemistry

Center for Biochemistry and Molecular Cell Biology

Georg-August-Universität Göttingen

Member of the Thesis Committee (Reviewer):

PD Dr. Wilfried Kramer

Department Molecular Genetics

Institute for Microbiology and Genetics

Georg-August-Universität Göttingen

Member of the Thesis Committee:

Prof. Dr. Marina Rodnina

Department of Physical Biochemistry

Max Planck Institute for Biophysical Chemistry, Göttingen

Date of oral examination: 03.09.2015 
Affidavit:

I herewith declare that this thesis has been written independently and with no other sources and aids than quoted.

Jan-Moritz Wuttke

Göttingen, 28.06.2015 



\section{Table of contents}

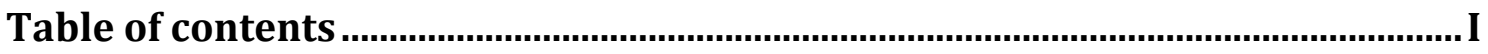

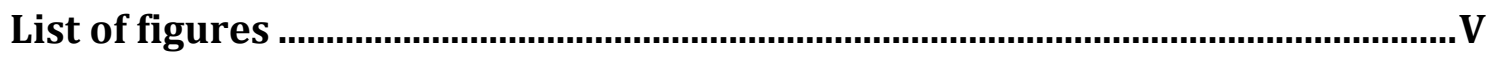

List of tables .........................................................................................................II

Abbreviations ….............................................................................................. IX

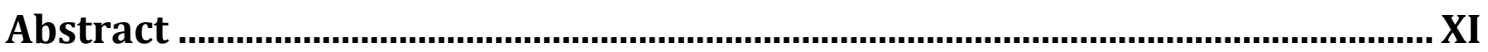

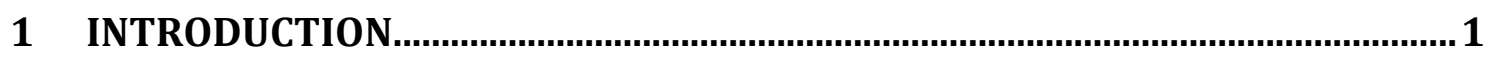

$1.1 \quad$ Mitochondria................................................................................................................... 1

1.1.1 Origin of mitochondria ............................................................................ 1

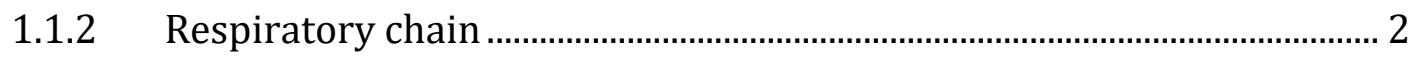

1.1.3 Additional roles of mitochondria .................................................................. 3

$1.2 \quad$ Mitochondrial Membrane transport................................................................... 3

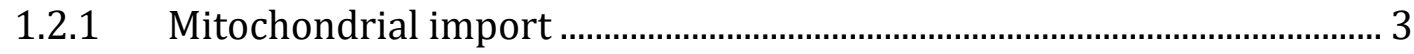

1.2.2 Export by 0xa1, Cox18 and Mba1 ……………………………………... 4

$1.3 \quad$ Mitochondrial translation......................................................................................... 6

1.3.1 Translational regulation in mitochondria ……………………………….. 7

1.3.2 Assembly-controlled translational auto-regulation of cytochrome $c$

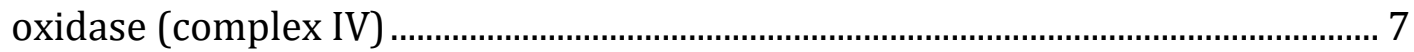

1.3.3 Regulation of translation in the context of complex III assembly .......... 8

1.3.4 Mitochondrial expressosome - MIOREX........................................................ 8

1.3.5 The mitochondrial ribosome ……………………………………………...

1.4 Mdm38 - A protein with multiple roles...............................................................11

1.4.1 Ylh47 and Ydl183c are functionally related to Mdm38...........................15

1.5 The Letm1-family ..........................................................................................16

1.5.1 Letm1 in the endoplasmic reticulum.........................................................17

$1.6 \quad$ Roles of Letm1 in human ....................................................................................17

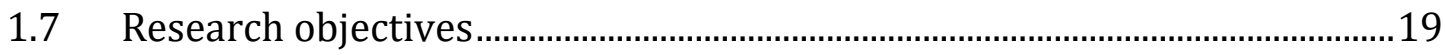

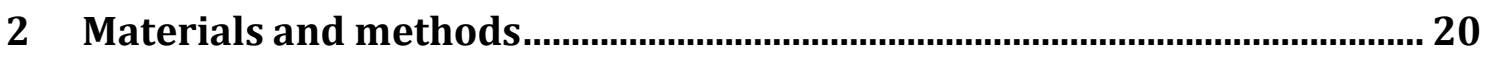

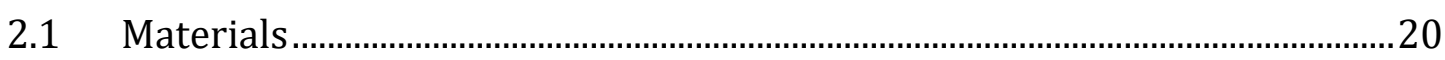

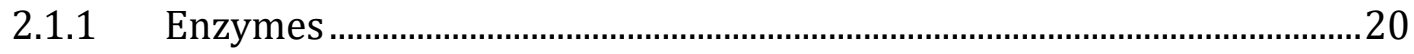

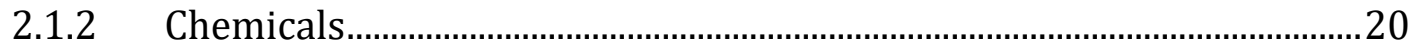

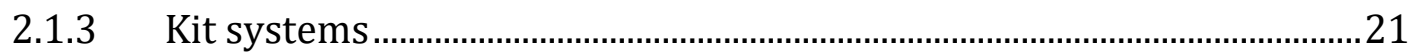

2.1.4 Antibodies …………………………………………………………………....21 


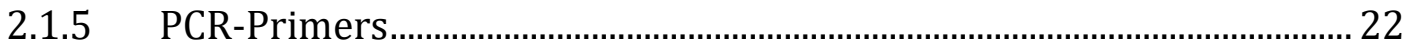

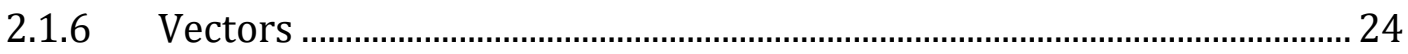

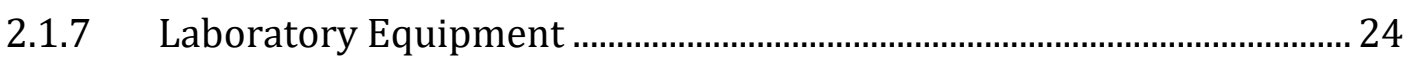

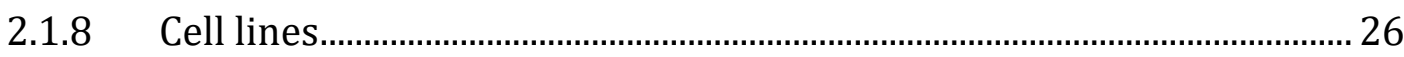

$2.2 \quad$ Growth conditions and media ......................................................................... 27

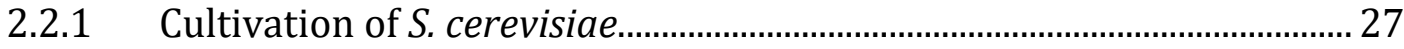

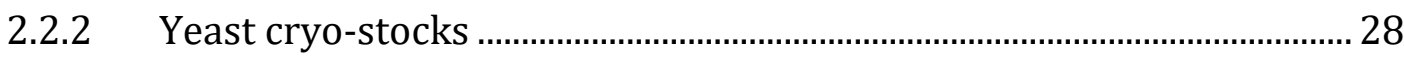

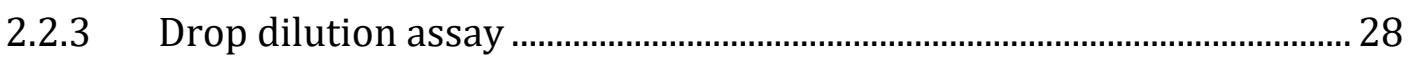

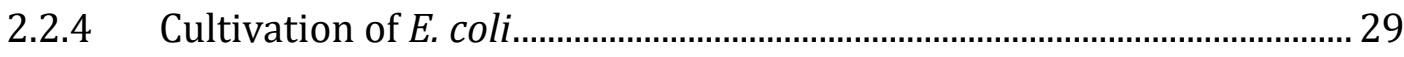

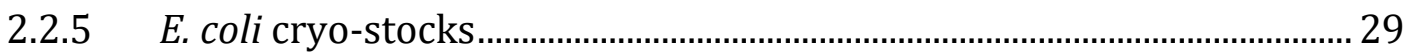

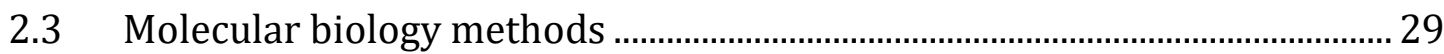

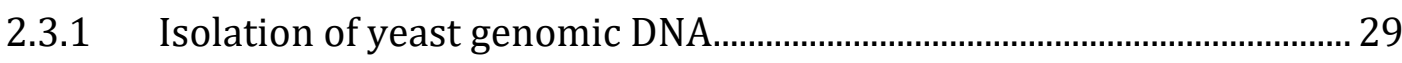

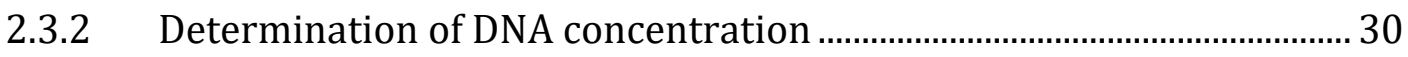

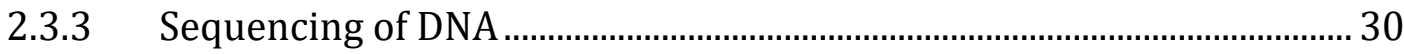

2.3.4 Agarose gel electrophoresis..................................................................... 30

2.3.5 Amplification of DNA fragments by PCR.................................................... 30

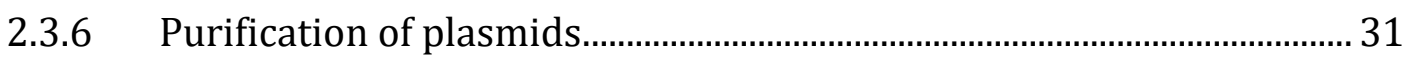

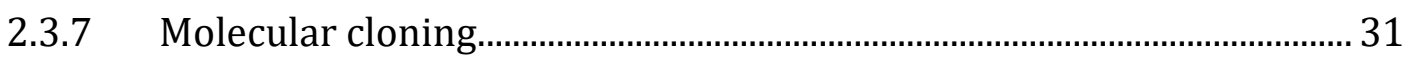

2.3.8 Site directed mutagenesis in plasmids ...................................................... 31

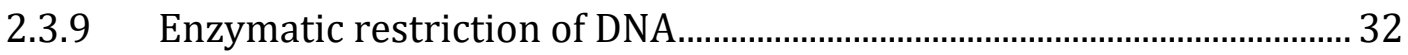

2.3.10 Transformation of yeast cells with lithium acetate .................................. 32

2.3.11 Preparation of transformation competent E. coli cells with divalent cations 33

2.3.12 Transformation of chemically competent E. coli ...................................... 34

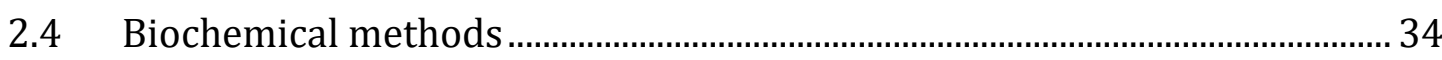

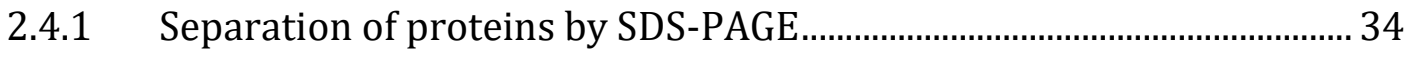

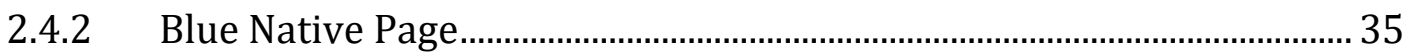

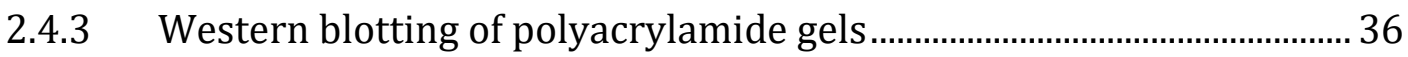

2.4.4 Immunostaining of Western Blot membranes ......................................... 37

2.4.5 Western Blot staining with tag-specific probes ...................................... 37

2.4.6 Coomassie staining of Western Blot membranes or polyacrylamide gels $\quad 37$

2.4.7 Colloidal coomassie staining of polyacrylamide gels ................................ 38

2.4.8 Preparation of colloidal Coomassie stock solution................................... 38 
2.4.9 Protein extraction from whole yeast cells ...............................................38

2.4.10 Determination of protein concentrations via Bradford assay ................38

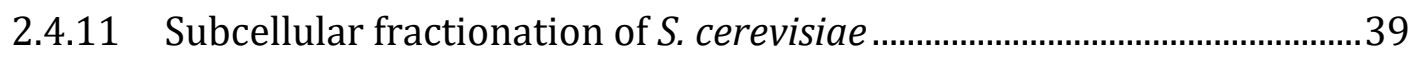

2.4.12 In vivo labeling of mitochondrial translation products ...........................40

2.4.13 Analysis of stability of mitochondrial translation products in vivo with pulse-chase experiments

2.4.14 In organello labeling of mitochondrial translation products 41

2.4.15 Stability analysis of mitochondrial translation products in organello via within pulse-chase experiments

2.4.16 Submitochondrial localization of proteins by protease protection.....42

2.4.17 Isolation of protein complexes using Protein A-tag..................................42

2.4.18 Activation and equilibration of IgG-Sepharose beads..............................43

2.4.19 Affinity purification with Glutathione Sepharose ....................................44

2.4.20 Mdm38K BpA in vivo cross-linking............................................................... 45

2.4.21 In vitro cross-linking to Mdm38K ................................................................ 46

2.4.22 Isolation of biotinylated proteins from mitochondria ..............................46

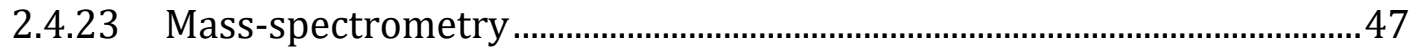

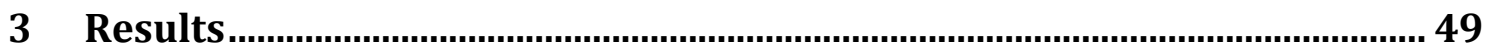

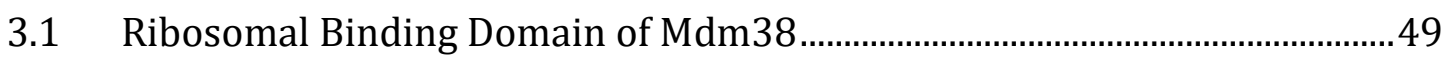

3.1.1 Binding to mitochondrial ribosomes in yeast is salt dependent...........49

3.1.2 Binding to E. coli ribosomes shows conserved characteristics ..............50

3.2 Establishing in vivo photo cross-linking with $\mathrm{pBpA}$..........................................53

3.2.1 Mdm38 derivatives with alanine exchanges are functional ...................54

3.2.2 Creation of an Mdm38K construct for pBpA incorporation ...................55

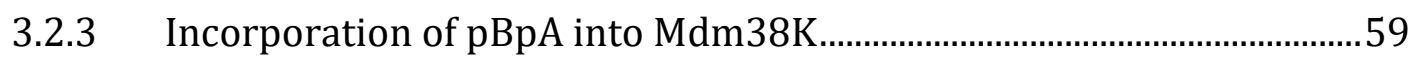

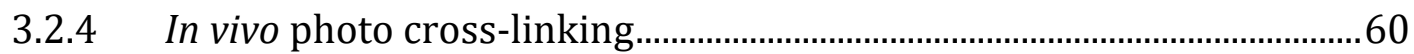

3.2.5 C315 of Mdm38 is accessible for maleimide-mediated in vitro

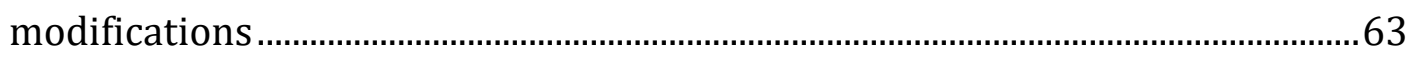

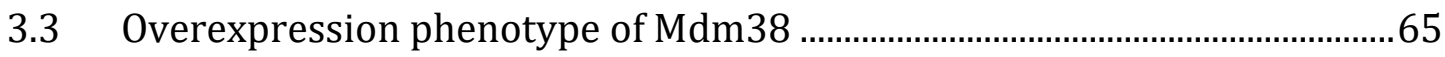

3.3.1 Suppression and creation of phenotypes by extra-chromosomally expressed Mdm38

3.3.2 Overexpression strains resemble the $m d m 38 \Delta$ phenotype on Western Blot and Blue Native PAGE.. . .66

3.3.3 Mdm38 overexpression does not affect translation .................................68

3.4 Spatially restricted enzyme mediated biotin tagging ...................................... 71 
3.4.1 Characterization of BirA-fusion strains..................................................... 71

3.4.2 Identification of biotinylated proteins......................................................... 73

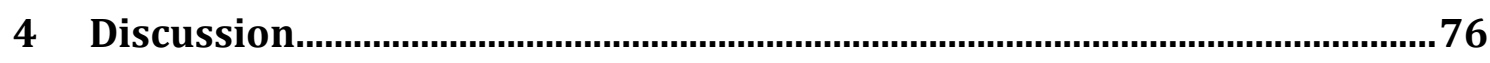

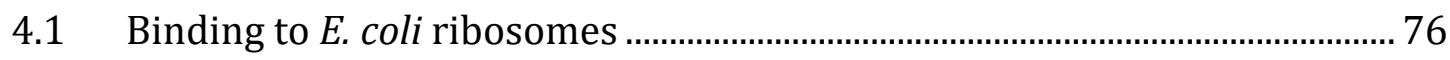

4.2 In vivo photo cross-linking with $\mathrm{pBpA}$........................................................... 79

4.3 Mdm38 neighboring proteins in S. cerevisiae .................................................... 83

4.4 Overexpression of $\mathrm{Mdm} 38$ induces a $m d m 38 \Delta$ like phenotype .................... 84

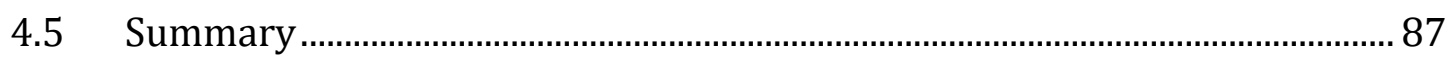

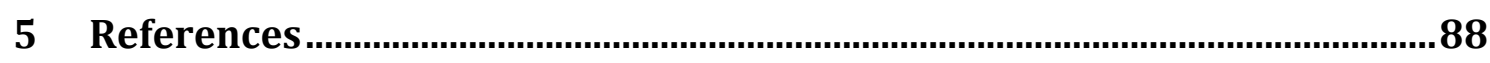




\section{List of figures}

Figure 1.1 The OXPHOS system of $S$. cerevisiae …........................................................... 3

Figure 1.2 Membrane transport in mitochondria ........................................................... 5

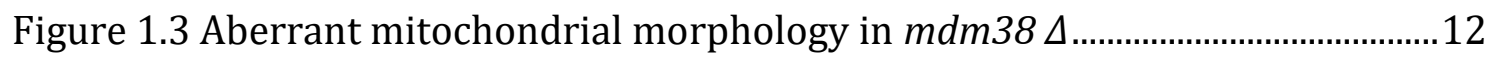

Figure 1.4 Structure of Mdm38 ribosomal binding domain ..........................................14

Figure 1.5 Model for the roles of Mdm38 and Mba1 in translation ..............................15

Figure 3.1 Salt-dependent co-isolation of mitochondrial proteins with Mdm38ProtA

Figure 3.2 Co-isolation of E. coli ribosomes using recombinant expressed constructs of Mdm38 51

Figure 3.3 Removal and rebinding of ribosomes to immobilized Mdm38K...............52

Figure 3.4 Salt-dependent binding to E. coli ribosomes ................................................53

Figure 3.5 Positions for pBpA incorporation.................................................................. 54

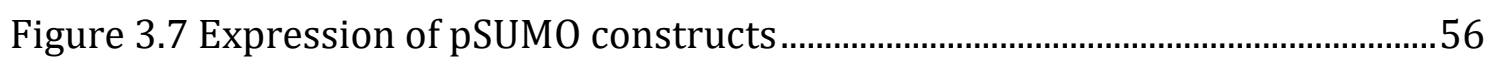

Figure 3.8 Cloning strategy for Mdm38K constructs ......................................................58

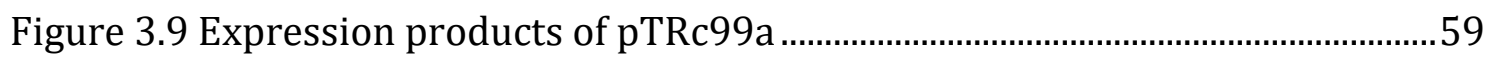

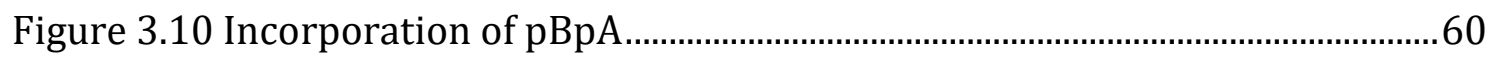

Figure 3.11 pBpA incorporation and photo cross-linking ..............................................61

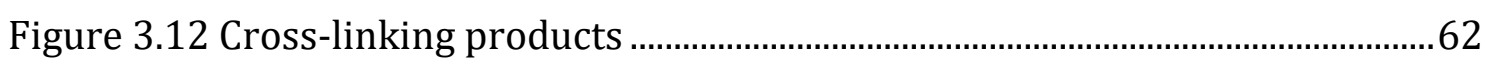

Figure 3.13 Modification of C315 of Mdm38K …............................................................64

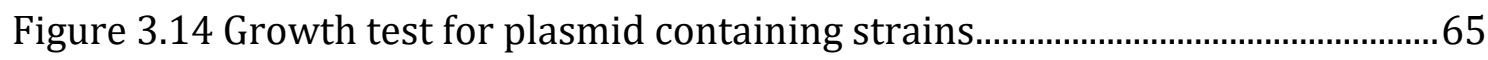

Figure $3.15 \mathrm{Mdm} 38$ overexpressing strains resemble a mild phenotype of $m d m 38 \Delta$ 68

Figure 3.16 Nigericin does not mitigate the growth phenotype ..................................69

Figure 3.17 In vivo labeled mitochondrial translation products are not affected in Mdm380Ex .69

Figure 3.18 Mitochondrial translation products are not destabilized in Mdm380Ex . .70

Figure 3.19 Schematic illustration of the biotinylation approach .71

BirA C-terminally fused to Mdm38 auto biotinylates Mdm38 and also proximal proteins in vivo (green lines). Mitochondria were subsequently isolated and lysed 
under denaturing conditions. Finally biotinylated proteins were isolated with

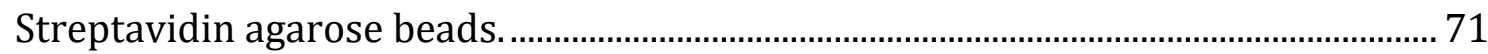

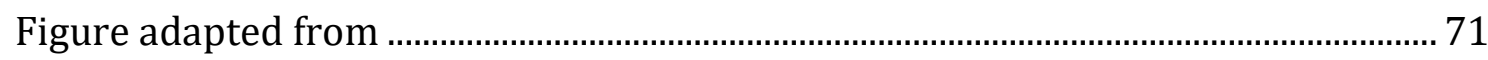

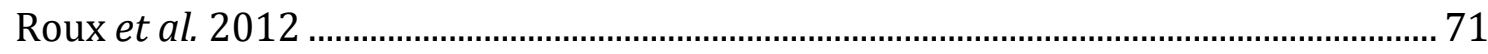

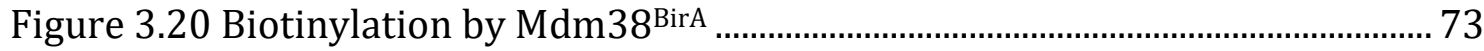

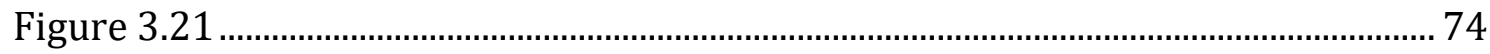

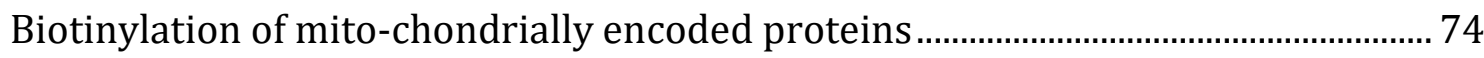

Figure 3.22 Streptavidin isolation for mass spectrometry ........................................... 75 


\section{List of tables}

Tab. 1.1 Mitochondrial translation activators (Kuzmenko et al. 2014) 7

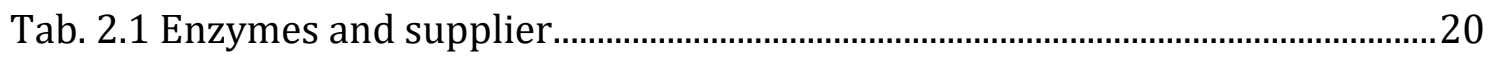

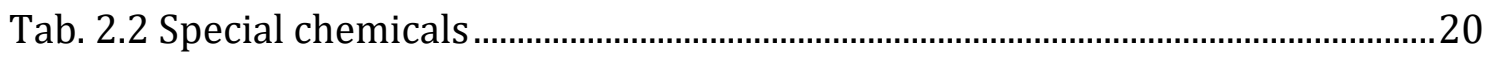

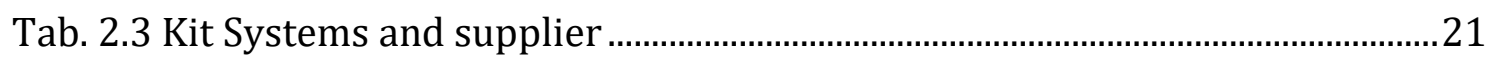

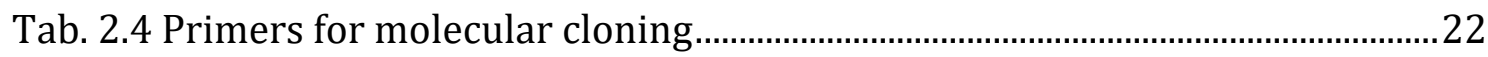

Tab. 2.5 Primers for site directed mutagenesis .................................................................. 23

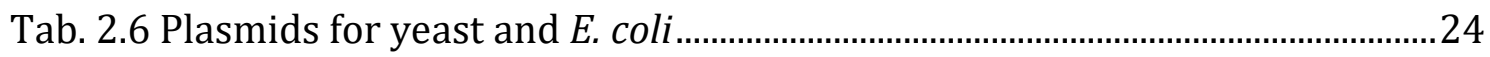

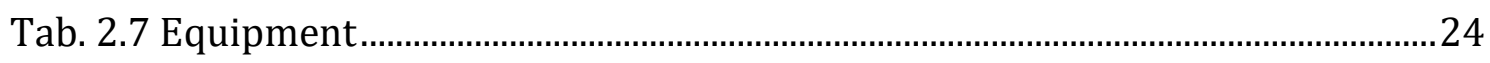

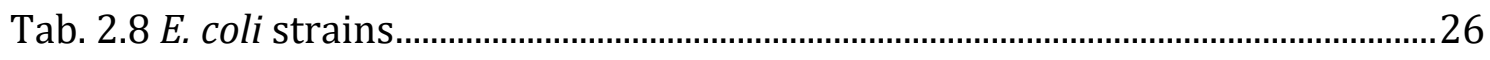

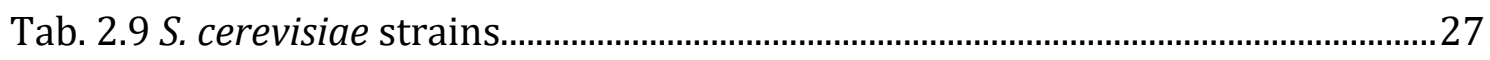

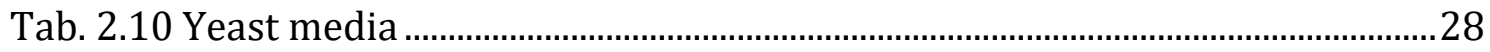

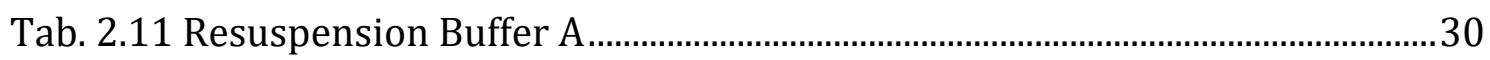

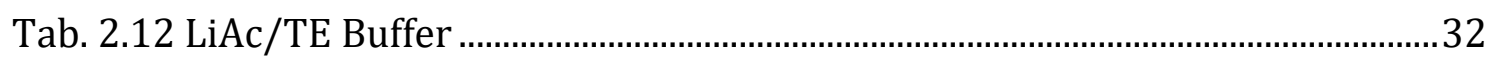

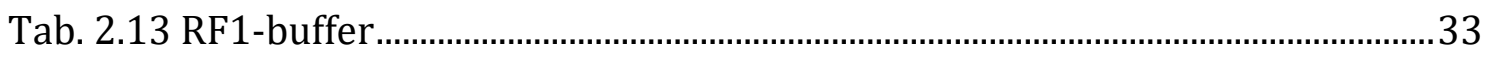

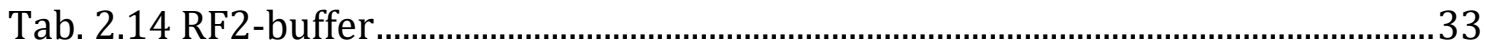

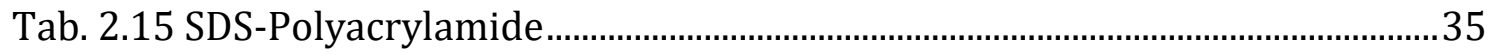

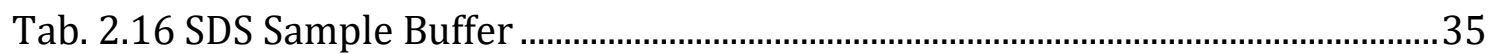

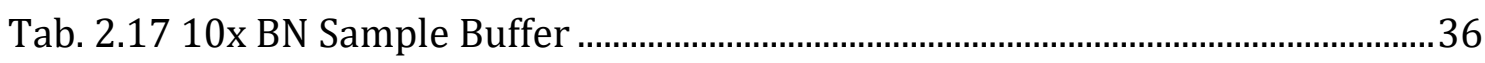

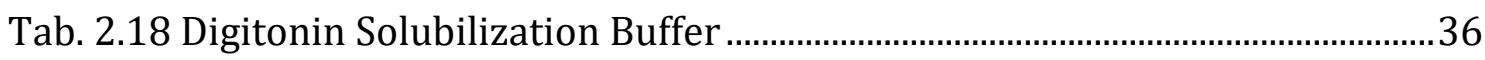

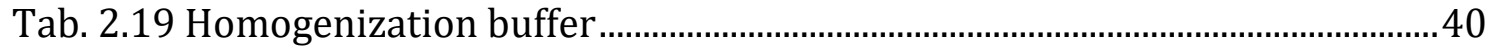

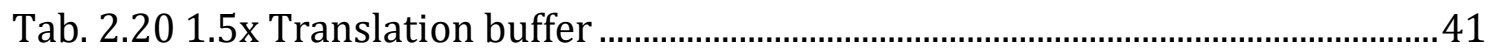

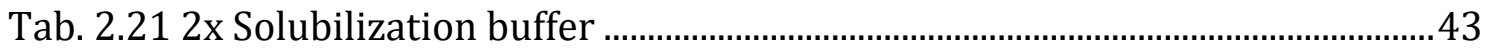

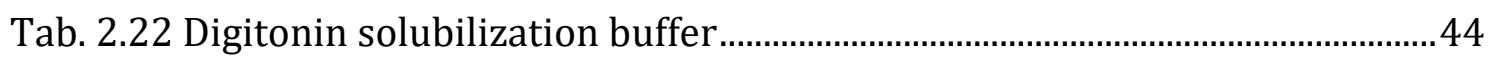

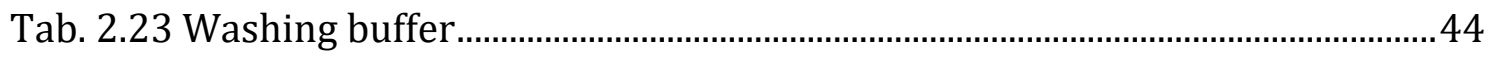

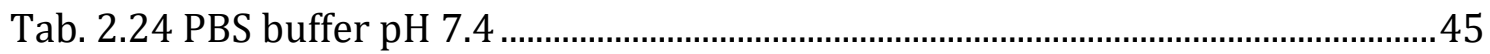

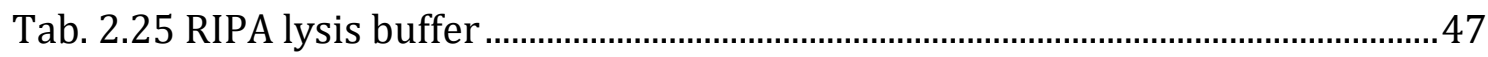

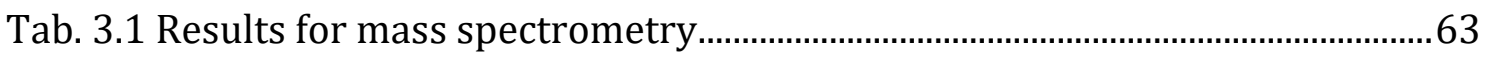


P a g e | VIII 


\section{Abbreviations}

\begin{tabular}{|c|c|}
\hline ADP & Adenosine diphosphate \\
\hline APEX & Ascorbate peroxidase \\
\hline ATP & Adenosine triphosphate \\
\hline$\AA$ & Ångström $\left(10^{-10} \mathrm{~m}\right)$ \\
\hline $\mathrm{BN}$ & Blue Native \\
\hline BSA & Bovine serum albumin \\
\hline${ }^{\circ} \mathrm{C}$ & Degree Celsius \\
\hline $\mathrm{CSM}$ & Complete supplement mixture \\
\hline DMSO & Dimethyl sulfoxide \\
\hline DNA & Deoxyribonucleic acid \\
\hline EDTA & Ethylenediaminetetraacetic acid \\
\hline $\mathrm{FADH}_{2}$ & Flavin adenine dinucleotide \\
\hline GSH & Reduced Glutathione \\
\hline GST & Glutathione S-transferase \\
\hline $\mathrm{h}$ & $\operatorname{Hour}(\mathrm{s})$ \\
\hline $\mathrm{HCl}$ & Hydchloric acid \\
\hline HRP & Horseradish peroxidase \\
\hline $\operatorname{IgG}$ & Immunoglobulin G \\
\hline IM & (Mitochondrial) inner membrane \\
\hline IMS & Intermembrane space \\
\hline IPTG & Isopropyl $\beta$-D-1-thiogalactopyranoside \\
\hline $\mathrm{kDa}$ & Kilodalton \\
\hline l & Liter \\
\hline M & Mol per liter \\
\hline $\mathrm{mCi}$ & Millicurie \\
\hline $\mathrm{mA}$ & Milliampere \\
\hline $\mathrm{mg}$ & Milligram \\
\hline $\min$ & Minute(s) \\
\hline $\mathrm{ml}$ & Milliliter \\
\hline $\mathrm{mM}$ & Millimol per liter \\
\hline MOPS & 3-(N-morpholino)propanesulfonic acid \\
\hline mRNA & Messenger ribonucleic acid \\
\hline NADH & Nicotinamide adenine dinucleotide \\
\hline
\end{tabular}




\begin{tabular}{|c|c|}
\hline $\mathrm{nm}$ & Nanometer \\
\hline OD & Optical density/absorbance \\
\hline $\mathrm{OM}$ & (Mitochondrial) outer membrane \\
\hline OXPHOS & Oxidative phosphorylation \\
\hline PAGE & Polyacrylamide gel electrophoresis \\
\hline $\mathrm{pBpA}$ & para-benzoylphenylalanine \\
\hline PBS & Phosphate buffered saline \\
\hline PCR & Polymerase chain reaction \\
\hline $\mathrm{P}_{\mathrm{i}}$ & Inorganic phosphate \\
\hline PMSF & Phenylmethylsulfonyl fluoride \\
\hline PVDF & Polyvinylidene fluoride \\
\hline RNA & Ribonucleic acid \\
\hline ROS & Reactive oxygen species \\
\hline rpm & Revolutions per minute \\
\hline $\mathrm{RT}$ & Room temperature \\
\hline RET & Ribosomal exit tunnel \\
\hline SDS & Sodium dodecyl sulfate \\
\hline$s$ & Second(s) \\
\hline SA & Streptavidin \\
\hline TBS & Tris buffered saline \\
\hline TCA & Trichloroacetic acid \\
\hline TIM & Translocase of the inner membrane \\
\hline TOM & Translocase of the outer membrane \\
\hline Tris & Tris(hydroxymethyl)aminomethane \\
\hline UTR & Untranslated region \\
\hline $\mathrm{V}$ & Volt \\
\hline WHS & Wolf-Hirschhorn syndrome \\
\hline$\Delta \psi$ & Membrane potential \\
\hline$\mu \mathrm{l}$ & Microliter \\
\hline$\mu g$ & Microgram \\
\hline$\%$ & Percent \\
\hline
\end{tabular}




\section{Abstract}

Mdm38 is a versatile protein from Saccharomyces cerevisiae, which is functionally involved in mitochondrial translation, export of mitochondrial proteins and potassium homeostasis of the organelle.

This thesis focuses on the investigation of interaction partners of the recently identified ribosomal binding domain of Mdm38. In previous studies, isolations of recombinant GST-tagged constructs of this domain from E. coli were found to exhibit an enrichment of ribosomal proteins and this binding showed conserved characteristics. To further define the molecular interactors of mdm38 in this thesis, an in vivo photo cross-linking approach, using the non-natural amino acid parabenzoylphenylalanine (pBpA), was established. In particular, different conserved amino acids within the presumed binding cavity of the domain were exchanged with $\mathrm{pBpA}$. The resulting $\mathrm{pBpA}$-specific cross-links were further characterized via mass spectrometry. Strikingly, results did not reveal any ribosomal proteins, but only transcription and translation related proteins, suggesting a connection to $E$. coli ribosomes.

In the second part of this thesis, the function of Mdm38 function in yeast was further characterized. It was shown that plasmid based expression of Mdm38 complements growth defects of Mdm38-truncation strains lacking the ribosomal binding domain. In addition, it was demonstrated that mild overexpression of Mdm38, using the multicopy plasmid Yep352, induces a phenotype resembling characteristics of the deletion strain. Since the stability and translation of mitochondria-encoded proteins was not changed, this suggests deficiencies in the assembly of complexes containing mitochondrial encoded subunits. Additional experiments excluded the possibility that the discovered phenotype was due to the role of Mdm38 in potassium homeostasis, since application of the artificial $\mathrm{K}^{+} / \mathrm{H}^{+}-$ exchanger, nigericin, mitigates the phenotype of the deletion mutant but not that of the overexpression strain.

Finally, another approach, using the biotin ligase BirA, was undertaken to identify proteins within the close environment of Mdm38 in yeast. BirA biotinylates proteins within 20-30 nm and was genetically fused to Mdm38. Thus, it was possible to visualize specific biotinylated proteins by Western Blot and to confirm their localization in the mitochondrial matrix. Indeed, preliminary mass spectrometry analysis was in line with pervious publications, serving as a proof of concept for this approach. 
P a g e | XII 


\section{INTRODUCTION}

\subsection{Mitochondria}

\subsubsection{Origin of mitochondria}

A giant leap in the evolution of eukaryotic life was the acquirement of cellular organelles. First theories for the origin of these cell components were already postulated more than a century ago, whereby an endosymbiotic process was hypothesized for the incorporation of chloroplasts (Schimper 1883). According to this endosymbiotic theory, plastids originate from prokaryotic cells, which were engulfed during non-digestive phagocytosis by a eukaryotic ancestor. The idea of this process was later also transferred to mitochondria, with genetic studies suggesting an aerobe $\alpha$-proteobacterium (Family: Rickettsiaceae) as a potential candidate incorporated by an anaerobic archeo-bacterium approximately 1,500 million years ago (Sicheritz-Pontén et al. 1998; Gray et al. 2001). In line with this theory, mitochondria still maintain a variety of prokaryotic features.

First of all, mitochondria are equipped with their own genetic information, encoded on circular DNA, which is translated by a mitochondria-specific translation system (Leon and Mahler 1968). However, most of the genetic information was transferred to the nuclear genome during evolution (Daley et al. 2002). This means only 8 proteins in S. cerevisiae and 13 proteins in human are mitochondrially encoded, whereas the majority of mitochondrial proteins originate from nuclear genes (Elstner et al. 2009). In consequence, mitochondria have to take up proteins, lipids and RNAs in order to fulfill their different biochemical functions in the cell.

Also, linked to the endosymbiotic event is the submitochondrial organization. Mitochondria are enclosed by two phospholipid membranes, each with a specific composition: the outer membrane (OM) and the inner membrane (IM). The IM is supposed to originate evolutionary from the plasma-membrane of the host cell, whereas the $\mathrm{OM}$ represents a relic of the endosymbionts coat. In this regard, it was shown that the lipid cardiolipin predominantly appears in bacterial cell membranes and in the IM (Hoch 1992; Mileykovskaya and Dowhan 2009). Accordingly, both membrane envelopes result in two aqueous compartments; 
Thereby, the mitochondrial matrix, which is enclosed by the IM, and the intermembrane space (IMS), which separates the inner from the outer membrane.

\subsubsection{Respiratory chain}

The most prominent advantage achieved by the described endosymbiosis of the $\alpha$ proteobacterium, is the exploitation of its aerobic lifestyle. In mitochondria, energy is provided by the oxidative phosphorylation (OXPHOS) of adenosine diphosphate (ADP). This process takes place at the mitochondrial IM and can be further divided into two processes. In a first step, reducing equivalents $\left(\mathrm{FADH}_{2} \& \mathrm{NADH}\right)$, generated by the catabolic metabolism of glucose and fatty acids, are oxidized. Electrons are then transferred through a redox system of complexes I-IV and two electron-shuttling entities (Ubiquinone \& Cytochrome c) within the membrane, and finally to oxygen. This latter process is termed the respiratory chain (Stuart and Rehling 2008). During the transfer of electrons through the respiratory chain, the pumping of protons into the IMS creates an electrochemical gradient $(\Delta \psi)$, which in turn is the driving force for the $\mathrm{F}_{1} \mathrm{~F}_{0}$-ATP-synthase (also termed complex V). This latter enzyme complex finally catalyzes the binding of free phosphate $\left(\mathrm{P}_{\mathrm{i}}\right)$ to ADP (Mills et al. 2006). Thus, catabolism is indirectly connected to the production of the universal cellular energy equivalent adenosine triphosphate, ATP. Interestingly, S. cerevisiae, the main model organism for studies investigating mitochondrial respiration, lacks complex I of the respiratory chain (Figure 1.1). In this organism, oxidation of NADH is facilitated by other NADH dehydrogenases (external: Nde1 \& Nde2, internal: Ndj1) (Boumans et al. 1998).

Since the respiratory chain is composed of different sub-components that could be crystallized as single complexes, a fluid model was proposed, describing separate units moving freely in the membrane (Hackenbrock et al. 1986). Later observations suggest a higher level of organization, since non-denaturing separation of mildly solubilized mitochondria by Blue Native-PAGE revealed different supercomplexes. These supercomplexes consist of complexes III and IV, which are composed in yeast as follows: a dimer of Complex III (III2) and two heteromers of complexes III and IV (III 2 IV \& III $I_{2} I_{2}$ ) (Schägger 2001). Additionally, a higher organization of complex $\mathrm{V}$ was identified as a homo-dimer $\left(\mathrm{V}_{2}\right)$ (Arnold et al. 1998). 


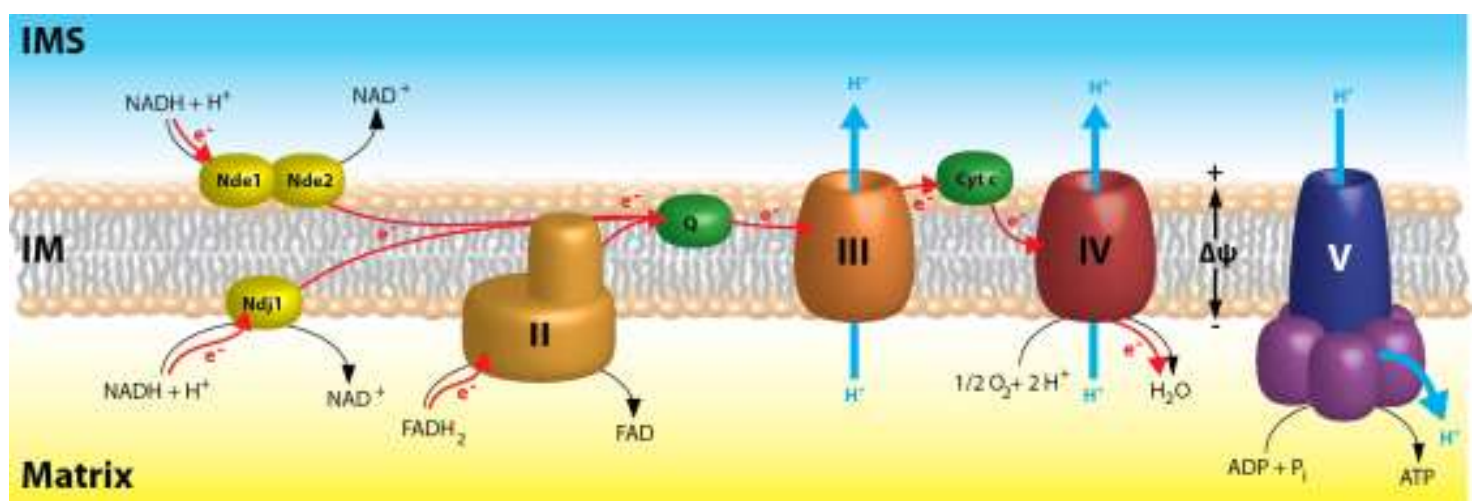

Figure 1.1 The OXPHOS system of $S$. cerevisiae

Schematic illustration of the mitochondrial oxidative phosphorylation system in yeast. Shown are the respiratory chain complexes II-IV and the F1FO-ATP synthase (V) in the inner mitochondrial membrane. NADH dehydrogenases are depicted in yellow and mobile electron shuttles Ubiquinone (Q) and Cytochrome $c$ (Cyt $c$ ) in green. The flux of electrons $(\mathrm{e}-)$ is indicated by red and the shuttling of protons $(\mathrm{H}+)$ by blue arrows.

\subsubsection{Additional roles of mitochondria}

Although mitochondria are predominantly known as the powerhouse of the cell, the organelle fulfils a wide spectrum of additional functions.

First of all, the iron-sulfur cluster biogenesis represents an even more important process than oxidative phosphorylation, since even non-respiring organisms retain this mitochondria-based process. Iron-sulfur clusters are cofactors not only for mitochondrial, but also a variety of cytosolic enzymes involving electron-transfer processes (Lill 2009).

Furthermore, in higher eukaryotes, mitochondria are involved in cell death mechanisms (Martinou and Youle 2011), as well as in calcium signaling and storage (Rimessia et al. 2008).

\subsection{Mitochondrial Membrane transport}

The above mentioned structural features, together with the fact that mitochondria contain proteins of dual genomic origin, require specialized systems for import and export, which are schematically summarized in Figure 1.2.

\subsubsection{Mitochondrial import}

The import of nuclear encoded proteins is channelled via the translocase of the outer membrane (TOM) and the translocase of the inner membrane (TIM23). Protein targeting to the mitochondria is facilitated predominantly by a presequence (Vögtle et al. 2009; Becker et al. 2012). Imported proteins are recognized at the cytosolic side of the outer membrane by three receptor 
components: Tom20, Tom22 and Tom70 (Komiya et al. 1997; Komuro et al. 2013). The translocation through the OM then takes place through a central proteinconducting channel of the TOM-complex, which is formed by the $\beta$-barrel protein Tom40 (Vestweber et al. 1989; Becker et al. 2005).

Upon import, presequence-containing proteins destined for the inner membrane or the mitochondrial matrix are handed over to the translocase of the inner membrane (TIM). TIM complexes can be further divided in two different forms, TIM23 ${ }^{\text {MOTOR }}$ and TIM23 $3^{\text {SORT }}$. These perform the translocation of precursors into the matrix and the membrane insertion of transmembrane domain-containing precursors (termed lateral sorting) respectively (Becker et al. 2012; Dudek et al. 2013). Full matrix translocation via the TIM23 ${ }^{\text {MOTOR }}$ requires its association with the presequence associated import motor (PAM). This complex drives inward motion of its substrate in an ATP-dependent process (van der Laan et al. 2010).

\subsubsection{Export by 0xa1, Cox18 and Mba1}

Following import of nuclear-encoded proteins into the mitochondrial matrix, proteins destined for the inner membrane are then exported from the mitochondrial matrix and translocated into the inner mitochondrial membrane. This involves the translocase Oxa1 (oxidase assembly mutant) and is closely coordinated with the TIM23 ${ }^{\text {MOTOR }}$ machinery (Reif et al. 2005; Bohnert et al. 2010). However, Oxa1 also facilitates the co-translational export of mitochondria-encoded proteins from the ribosome into the inner membrane. Studies have revealed an interaction of Oxa1 with newly synthesized mitochondrial proteins and also with mitochondrial ribosomes (Hell et al. 2001; Jia et al. 2003; Jia et al. 2009). Connected to its role in membrane translocation, Oxa1 was shown to form a pore with ion channel activity in the presence of a substrate peptide (Krüger et al. 2012).

Since Oxa1 mediated export shows highly conserved characteristics of the bacterial translocase machinery, this process is also termed conservative sorting. In bacteria, translocation is performed by YidC in order to secrete soluble proteins or insert proteins into the plasma membrane, (Bonnefoy et al. 2009; Park and Rapoport 2012).

An additional homologue of Oxa1 in yeast, and therefore also a member of the YidC/Alb3/Oxa1 family, is Cox18. Interestingly, Cox18 is involved in the export of 
the C-terminus of Cox2, while Oxa1 facilitates the export of the $\mathrm{N}$-terminus (Saracco and Fox 2002).

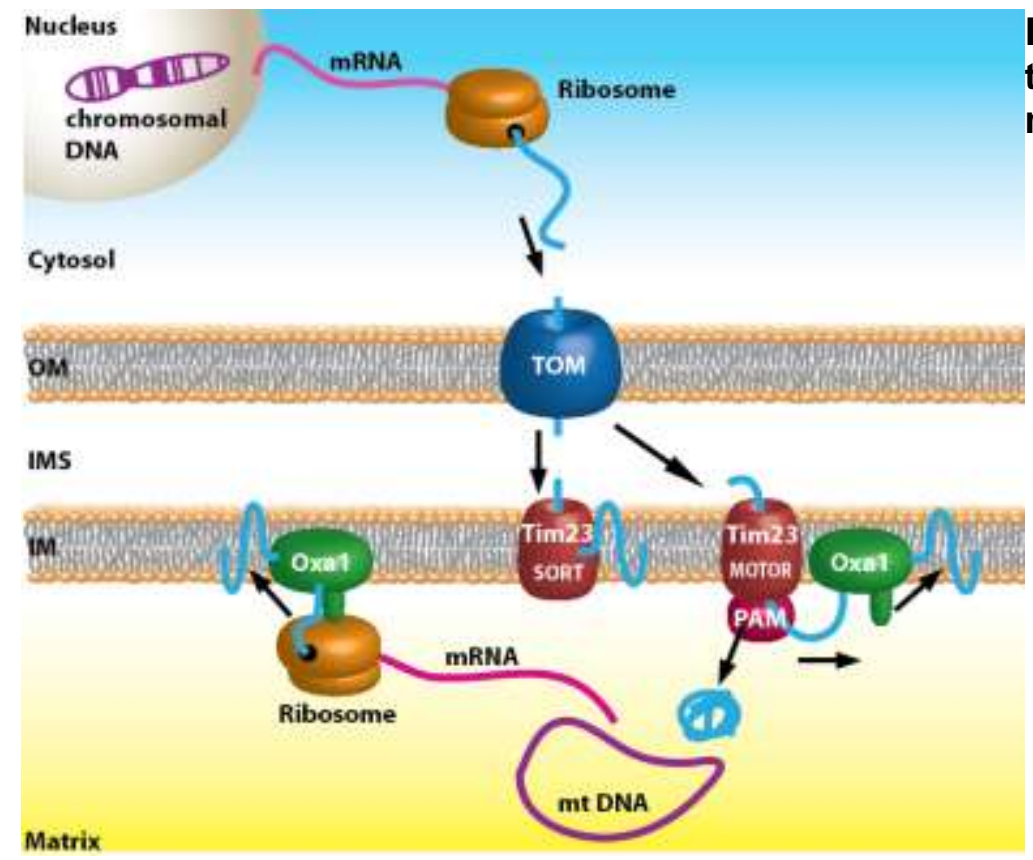

Figure 1.2 Membrane
transport in
mitochondria

Schematic illustration of the mitochondrial trans-port system relevant for proteins within the inner mitochondrial membrane and matrix. Transport pathways of newly synthesized proteins (light blue) are indicated by arrows.

Adapted from

Mick et al. 2011

Another protein involved in export of mitochondria-encoded proteins from the ribosome into the inner membrane is the inner membrane protein Mba1. The yeast Mba1 deletion mutant is deficient in the biogenesis of Cox2 and the membrane insertion of other mitochondria-encoded proteins, thereby implicating an involvement for Mba1 in translocation (Preuss et al. 2001). Moreover, an interaction with the mitochondrial ribosome has been shown, suggesting a ribosomal receptor function for Mba1 (Pfeffer et al. 2015). It was therefore postulated that Mba1 is involved in the positioning of the ribosomes at the inner membrane to facilitate co-translational hand-over of newly synthesized proteins to the export machinery. However, it is worth noting, that deletion of MBA1 and MDM38, encoding a protein implied in similar function (see 1.4), did not significantly change the membrane association of ribosomes in flotation centrifugation experiments (Bauerschmitt et al. 2010). Similar observations were also made by Ott et al. (2006) for deletions of MBA1 and the ribosome binding Cterminus of Oxa1. Thus, all three proteins are not critical in this regard. Nevertheless, membrane association is a prominent feature of mitochondrial ribosomes (Pfeffer et al. 2015; Beckmann and Herrmann 2015). This fact is most 
probably owed to their specialized role in the translation of mainly highly hydrophobic membrane proteins and thus the co-translational export of newly synthesized mitochondria-encoded proteins is required.

\subsection{Mitochondrial translation}

While import provides the majority of proteins that comprise the respiratory chain, respiratory chain biogenesis still depends on mitochondrial translation products. This is based on the fact that 7 out of 8 mitochondria-encoded proteins represent core subunits of complexes III-V in yeast. As is the case for it's prokaryotic ancestors, protein biosynthesis in mitochondria is performed in four steps; initiation, elongation, termination, and recycling. (Marshall et al. 2008). Accordingly, components of the mitochondrial translation machinery are distinct from their cytoplasmic counterparts, with the exception of three aminoacyl tRNA synthetases.

Initiation of translation is facilitated by two intiation factors, mIF2 \& mIF3, which both have bacterial orthologs. In yeast mIF2 recruits the initiator tRNA, preferably in its formylated form (fMet-tRNA $\mathrm{f}^{\mathrm{Met}}$ ), to the initiation codon of the mRNA on the ribosome (Gualerzi and Pon 1990; Garofalo et al. 2003). Meanwhile, mIF3 (S. cerevisiae: Aim23) is required for the dissociation of the ribosome into its subunits and the binding of the initiator tRNA to the ribosomal initiation complex (Koc and Spremulli 2002; Atkinson et al. 2012).

Translation after initiation is further enabled by three elongation factors. First, mEF-Tu promotes the GTP-dependent binding of an aminoacyl-tRNA to the A site of the ribosome (Kaziro 1978; Nagata et al. 1983). The factors mEF-G1 and mEF-G2 then facilitate the exchange of tRNAs at the ribosomal A-site and are involved in conformational changes in the ribosome that enable elongation of the peptide chain (Vambutas et al. 1991).

Finally, termination is facilitated by the mitochondrial release factor, $\mathrm{mRF}-1$, by a yet unknown mechanism (Towpik et al. 2004), along with the mitochondrial ribosome recycling factor Rrf1 (Teyssier et al. 2003). As described for bacterial recycling factors before (Selmer et al. 1999), Rrf1 dissociates the two ribosomal subunits in order to release the peptide chain and enable further rounds of translation. Rrf1 is suggested to fulfill its function by structural mimicry of tRNA (Teyssier et al. 2003).

P a g e | 6 


\subsubsection{Translational regulation in mitochondria}

In addition to the above described mechanisms of mitochondrial translation, this process requires further regulation for proteins of the respiratory chain complexes, due to their dual genetic origins. Thereby, in yeast, both genetic systems are coordinated by different mRNA-specific activators (listed in Tab. 1.1). The recognition of respective mRNAs is enabled by specific $5^{\prime}$ untranslated regions (UTRs).

Tab. 1.1 Mitochondrial translation activators (Kuzmenko et al. 2014)

\begin{tabular}{|l|l|}
\hline Target mRNA & Translational activators \\
Var1 & Sov1 \\
Cox1 & Cbs1, Cbs2, Cbp1, Cbp3/Cbp6 \\
Cox2 & Mss51, Pet309 \\
Cox3 & Pet111 \\
Atp6/8 - bicistronic & Pet54, Pet122, Pet494 \\
Atp9 & Atp22 \\
\hline
\end{tabular}

\subsubsection{Assembly-controlled translational auto-regulation of cytochrome $c$ oxidase (complex IV)}

The translational activators Pet309 (petite phenotype) and Mss51 (mitochondrial splicing suppressor protein) are essential for translation of Cox1 (cytochrome c oxidase). Genetic studies revealed a COX1-mRNA stabilizing function for Pet309, in addition to its UTR-dependent role in translational activation (Manthey and McEwen 1995; Tavares-Carreón et al. 2008).

In this respect, translational activation of COX1-mRNA, via Mss51, was also shown to occurby UTR-specific binding (PerezMartinez et al. 2003; Zambrano et al. 2007). In addition, further results suggested a mechanism, which is autoregulated by feedback from the assembly of Cox1 (Barrientos et al. 2004; Fox et al. 2009). In line with this, Mss51 interacts dynamically with Cox1 and several assembly factors of complex IV. Newly synthesized, unassembled Cox1 is bound by Mss51 in a transient pre-assembly complex, stabilized by the assembly factors Cox14 and Coa3 (cytochrome $c$ oxidase assembly) (Barrientos et al. 2004; Mick et al. 2010). Bound Mss51 is prevented from promoting further rounds of translation by its association with another assembly factor, Coa1. As Cox1 assembley proceeds, 
Mss51 is released from the complex, thereby enabling its reactivation. In summary, these processes directly connect the translation of Cox1 to its assembly (Barrientos et al. 2004; Mick et al. 2010), which is in turn also dependent on the translation and maturation of Cox2 \& Cox3 (Horan et al. 2005; Mick et al. 2010).

Overall, the assembly of complex IV in yeast, consisting of three mitochondrial and eight nuclear encoded subunits, involves approximately 40 currently identified nuclear-encoded proteins, starting with translation (Soto et al. 2012).

\subsubsection{Regulation of translation in the context of complex III assembly}

In contrast to complex IV, knowledge about the biogenesis of complex III is still scarce. The core subunit of the complex is cytochrome $b$ (Cob). Its mitochondrial translation is regulated in a UTR-dependent way by Cbs1, Cbs2 and Cbp1 (Rödel 1986; Krause-Buchholz et al. 2005). Additionally, a similar assembly-controlled translational auto-regulation, as described for Cox1, was also suggested for Cob. In this regard, the hetero-dimer of $\mathrm{Cbp} 3 \& \mathrm{Cbp} 6$ (Cbp3-Cbp6), bound to the ribosomal exit tunnel, is essential for the translation of Cob, where it subsequently binds to the newly synthesized protein. Cbp3-Cbp6 stays trapped in this preassembly complex with Cob and can only rebind to the ribosome after its release as complex III assembly proceeds (Gruschke and Ott 2010; Gruschke et al. 2011; Gruschke et al. 2012; Hildenbeutel et al. 2014)

\subsubsection{Mitochondrial expressosome - MIOREX}

Since ATP-synthesis is the result of the concerted interplay of different complexes, which in turn are also organized on even higher levels, a similar organization of their biogenesis is not unreasonable. Interestingly, this idea was supported by coimmunoprecipitation experiments and Yeast Two-Hybrid analysis. These data indicated a physical interaction, not only between the translational activators for Cox1, Cox2 and Cox3, but also to the ribosome and to Nam1, a protein involved in COX1-, COB- and ATP8/ATP6-mRNA interactions (Wallis et al. 1994; Naithani et al. 2003). Accordingly, Nam 1 is also locally associated with the mRNA-polymerase, Rpo41 (Rodeheffer et al. 2001). Moreover, mass spectrometry results for a high molecular weight complex revealed Pet309 and Cbp1 (Krause et al. 2004). These results were recently supported by mass spectrometric analysis of the interactome of the mitochondrial ribosome (Kehrein et al. 2015), suggesting expressosome-like 
assemblies that were termed MIOREX (mitochondrial organization of gene expression) complexes. In line with previous studies, most of the translational activators were herein identified. Furthermore, many proteins were recovered with ribosomes that are involved in mRNA maturation and processing, DNAmetabolism and organization of the mitochondrial nucleoid, as well as OXPHOSassembly factors (e.g. Oxa1). Thus, MIOREX complexes might organize mitochondrial gene expression by coupling translation of the mRNAs and posttranscriptional mRNA metabolism, with mRNA decay.

\subsubsection{The mitochondrial ribosome}

According to the endosymbiotic theory, mitochondrial ribosomes developed from those of the bacterial ancestor of the organelle. In this context, shared antibiotic susceptibilities and sequence similarities of ribosomal proteins and RNAs (rRNAs) suggests that the mitochondrial translation system is essentially identical to that of prokaryotes (Borst and Grivell 1971; Jones et al. 2009). However, mitochondrial ribosomes were significantly remodeled during evolution, since a variety of ribosomal proteins represent mitochondria-specific subunits and also the rRNA content is significantly reduced.

Altogether, comparisons of bacterial (E. coli) and yeast mitochondrial ribosomes has revealed a significant shift in the protein to nucleic acid ratio from 1:2 in bacteria to 1:1 in mitochondrial ribosomes (Graack and Wittmann-Liebold 1998; Smits et al. 2007). This is also reflected by the fact that mitochondrial proteins of the large subunit are, on average, nearly twice as large as their homologous bacterial counterparts. However, most of these new structural elements are exposed to the surface and thus, result in a markedly different overall ribosomal shape (Amunts et al. 2014).

Additionally, the reduced rRNA content in mitochondrial ribosomes contributes to the changed ratio. Correspondingly, the sedimentation coefficients $(S)$ of both rRNAs of the mitochondrial ribosome, $15 \mathrm{~S}$ and $21 \mathrm{~S}$ for the small and large subunit rRNA respectively, are lower than that of their bacterial counterparts, $16 \mathrm{~S}$ and $25 \mathrm{~S}$ respectively (Smits et al. 2007). Moreover, this shift from RNA to protein is nicely illustrated by the complete lack of $5 \mathrm{~S}$ rRNA of bacterial and eukaryotic cytosolic ribosomes in mitochondria in which its location is structurally occupied by mitochondria-specific protein-extensions (Amunts et al. 2014). 
Another hint that underlines the ongoing independent evolution of mitochondrial ribosomes is given by the fact that their composition already deviates between different species. This is exemplified by the high variation in mitochondrial ribosome mass and its corresponding sedimentation behavior which ranges from $55 \mathrm{~S}$ to $80 \mathrm{~S}$. S. cerevisiae is positioned in the midway within this range, with a mitochondrial ribosome of $74 \mathrm{~S}$, composed of a small $37 \mathrm{~S}$ and a large $54 \mathrm{~S}$ subunit (Kitakawa and Isono 1991; Smits et al. 2007)

Interestingly, the catalytic center of the ribosome was mainly untouched during evolution, since rRNA domains involved in peptide bond formation reveal high levels of conservation (Ott and Herrmann 2010; Amunts et al. 2014).

\subsubsection{Ribosomal exit tunnel}

The afore mentioned evolutionary changes in mitochondrial ribosomes also resulted in a remodeling of their polypeptide exit tunnel, leading to a unique architecture and composition.

The ribosomal exit tunnel (RET) contains, in addition to four ubiquitously conserved proteins (Mrpl4, Mrpl22, Mrpl40 \& Mrp20), at least three organellespecific proteins (Mrpl3, Mrpl13 \& Mrpl27) which are, with the exception of Mrpl13, conserved between mammals and yeast (Gruschke et al. 2010).

Due to the fact that RET is the site where nascent polypeptides are exposed to a hydrophilic environment for the first time, it is also the site where components of their assembly machinery are most likely positioned. In this respect, Oxa1 and Mba1 of the export machinery (1.2.2), and the assembly/translation regulator, Cbp3-Cbp6 (1.3.3) have been identified here (Gruschke et al. 2011; Gruschke et al. 2012; Pfeffer et al. 2015).

Interestingly, recent results based on cryo electron microscopy enabled further insights into the RET structure (Amunts et al. 2014). Although overall features of the tunnel are conserved, Amunts et al. revealed an alternate path that deviates approximately $60 \AA$ from that of the bacterial RET. In consequence, the ribosomal tunnel emerges nearly $35 \AA$ away from its evolutionary origin.

In summary, these features are unique for mitochondria with their own mechanisms of regulation and co-and post-translational interactions. 


\subsection{Mdm38 - A protein with multiple roles}

As discussed above, distinct mitochondrial functions have been shown to have some interrelations and so it follows that proteins may have multiple roles within the mitochondria. One such example is the evolutionarily conserved $65 \mathrm{kDa}$ protein Mdm38, which not only shows an involvement in the biogenesis of the respiratory chain, but also in mitochondrial $\mathrm{K}+/ \mathrm{H}+$-homeostasis.

Mdm38 was first characterized in a genome wide screen for genes important for mitochondrial distribution and morphology (MDM) in S. cerevisiae (Dimmer et al. 2002). This screen, from which Mdm38 derives it's name, revealed enlarged mitochondria, with very few branches, that often form rings or lariat-like structures in yeast cells lacking the Mdm38 protein (Figure 1.3).

Further studies confirmed the localization of Mdm38 at the matrix side of the inner mitochondrial membrane (Nowikovsky et al. 2004) and a single trans-membrane domain (TM) was predicted for all homologs of Mdm38. Full sequence alignments of different Mdm38 homologs indicated a highly conserved area around this TM domain (Schlickum et al. 2004; Nowikovsky et al. 2004). Since this proline-rich predicted alpha-helix alignes with that of other proteins involved in ion channels (Sansom and Weinstein 2000), Nowikowski et al. assumed a related function as a possible explanation for the aberrant mitochondrial morphology. Following this idea, the group revealed a role for Mdm38 in the maintenance of $\Delta \psi$ (Nowikovsky et al. 2004). In addition, experiments with fluorescent dyes implicated an involvement in potassium-homeostasis by electro neutral $\mathrm{K}^{+} / \mathrm{H}^{+}$-exchange. This was further supported by the efficient reversal of mitochondrial swelling and the restoration of an almost normal mitochondrial network upon addition of the $\mathrm{K}^{+} / \mathrm{H}^{+}$ ionophore nigericin (Nowikovsky et al. 2004; Froschauer et al. 2005). 


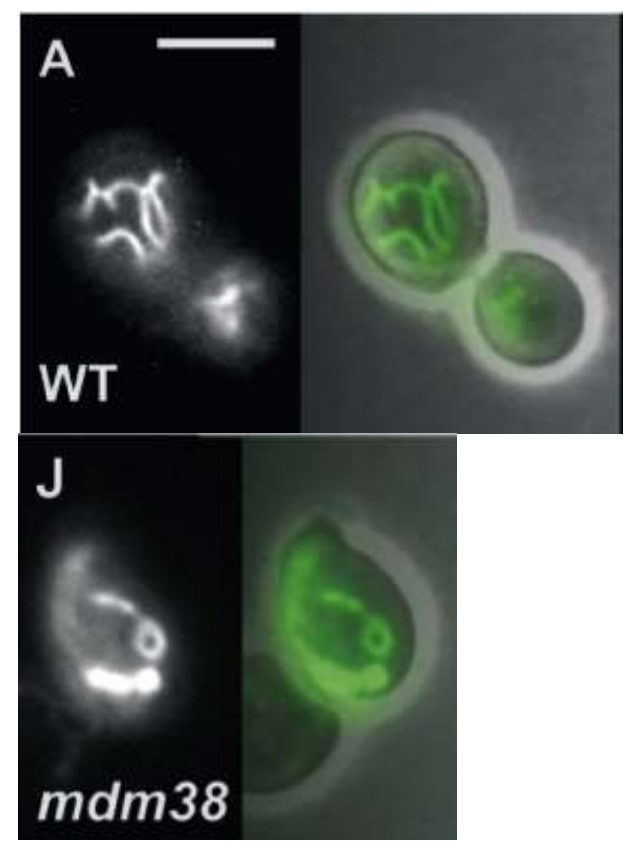

Figure 1.3 Aberrant mitochondrial
morphology in $\mathrm{mdm} 38 \Delta$

Shown are fluorescence microscopic images of the mitochon-drial morphology (left panel) and an overlay with the corresponding phase contrast image (right panel). For labeling, wild-type (WT) and MDM38 deletion strains (mdm38) expressing mitochondria-targeted GFP were grown at $30^{\circ} \mathrm{C}$ in glucosecontaining YPD medium to logarithmic growth phase.

Pictures taken from

Dimmer et al. 2002

Nevertheless, a single TM is unlikely to function as an ion-channel. In addition, only partial release of Mdm38 via carbonate extraction argues against a complete penetration of the inner membrane (Frazier et al. 2006). Therefore, these distinct functions of $\mathrm{Mdm} 38$ came into question. Interestingly, $m d m 38 \Delta$ yeast strains revealed severe growth deficiencies on non-fermentable media, indicating aberrations in the respiratory chain (Frazier et al. 2006). Correspondingly, $m d m 38 \Delta$ mitochondria showed a significant reduction in the steady-state levels of the mitochondrially encoded proteins $\operatorname{Cox} 1, \operatorname{Cox} 2$, and Cob, a slight reduction in Cox3 and a significant reduction in levels of the nuclear encoded Rieske protein (Rip1). Moreover, Blue Native PAGE analysis revealed deficiencies in respiratory chain biogenesis with significantly decreased levels of complexes III and IV and a slight decrease of complex V (Frazier et al. 2006). Since mitochondrial translation was nearly unchanged, those defects are expected to occur post-translationally. In line with this, co-isolations indicate that Mdm38 interacts specifically with mitochondrial ribosomes and newly synthesized mitochondrially encoded proteins. Moreover, proteinase protection assays revealed deficiencies in the posttranslational export of Atp6, Cob, and to a lesser extent, Cox1 in $\Delta m d m 38$ mitochondria. These results suggest a role for Mdm38 in an 0xa1-independent export pathway (Hell et al. 1998; Frazier et al. 2006) .

The two functions of Mdm38, ion-channeling and respiratory chain biogenesis, was further investigated by Nowikovsky et al. (2007). In this study, mitochondrial 
fragmentation upon Mdm38 shut-off and the association of mitochondrial fragments with vacuoles, suggests the occurrence of mitochondria-selective autophagy, termed mitophagy. Furthermore, mitophagy, was thought to be the primary phenotypical effect caused by diminished $\mathrm{K}^{+} / \mathrm{H}^{+}$exchange activity (Nowikovsky et al. 2007).

In contrast, Bauerschmitt et al. (2010) assessed the role of Mdm38 in respiratory chain biogenesis in a more independent context. In this study, a double deletion of MDM38 and MBA1 selectively prevented the synthesis of Cox1 and Cob. On one hand, this indicates an overlapping function of both proteins in translation. On the other hand, failed rescue of the $\Delta m b a 1 \Delta m d m 38$ phenotype by nigericin argues against a direct coupling to Mdm38's role in $\mathrm{K}^{+} / \mathrm{H}^{+}$exchange. Moreover, a physical interaction between Mdm38 and the translational activators Pet309 and Mss51 (see 1.3.1) was indicated by coisolations (Bauerschmitt et al. 2010; Pfeffer et al. 2015). Correspondingly, the reported dependency of Cox1 synthesis on Mba1 and Mdm38 was lost when the COX1 reading frame was flanked by the UTRs of COX2. In summary, results of Bauerschmitt et al. imply a functional role of Mba1 and Mdm38 in mitochondrial translation, possibly through the recruitment of specific translation activators.

Finally, the identification of a ribosomal binding domain (RBD, here also referred as Mdm38K) further manifested Mdm38 as a player in mitochondrial translation (Lupo et al. 2011). Herein, an area (aa 182-408) within the C-terminal domain (CTD) of Mdm38 was shown to interact with mitochondrial ribosomes. Interestingly, yeast strains expressing truncations lacking this RBD exhibit deficiencies in the synthesis of Cox 1 and $\operatorname{Cob}$ and, in consequence, also in the respiratory chain. However, the correlated growth phenotype is not mitigated by nigericin and thus not essentially dependent upon $\mathrm{K}^{+} / \mathrm{H}^{+}$exchange.

Additionally, Lupo and Vollmer et al. determined the structure of the RBD via X-ray crystallography (Figure 1.4). Surprisingly, this structure superimposes with 14-3-3 domains involved in different protein-protein interactions. Moreover, Mdm38K aligns with those 14-3-3 domains, since it possesses a highly conserved cavity assumed as a potential binding site. 
A)
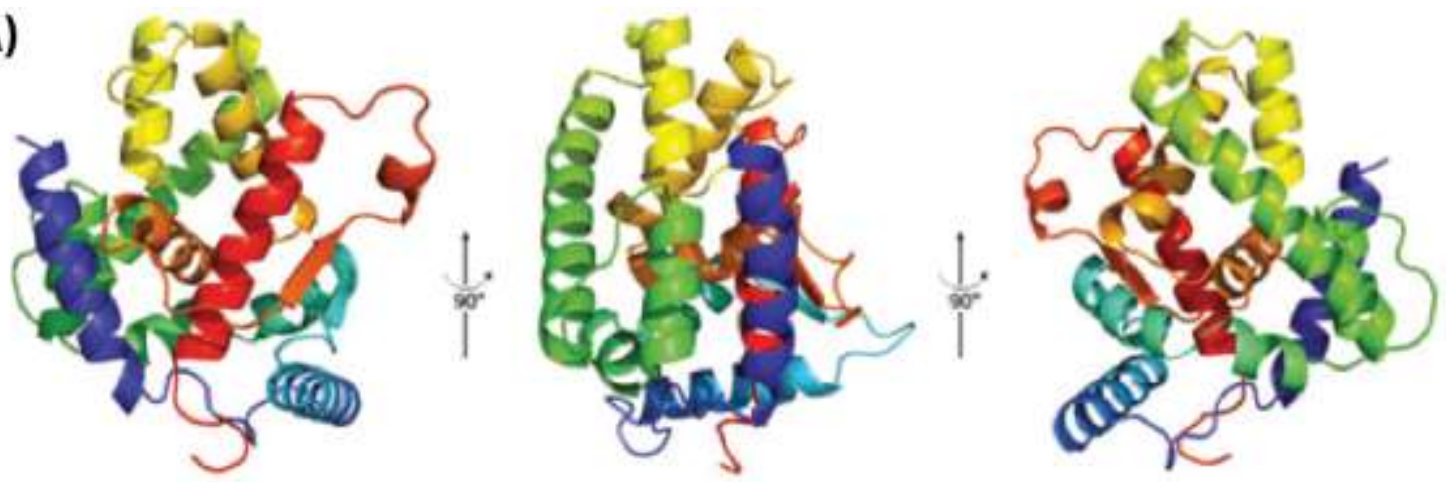

B)
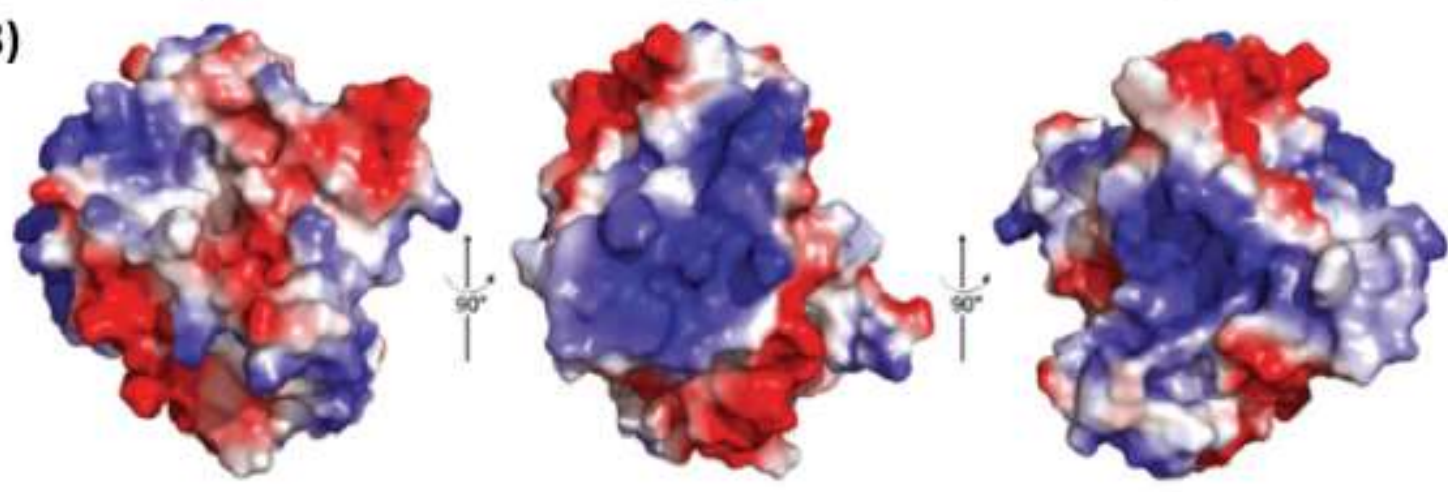

C)

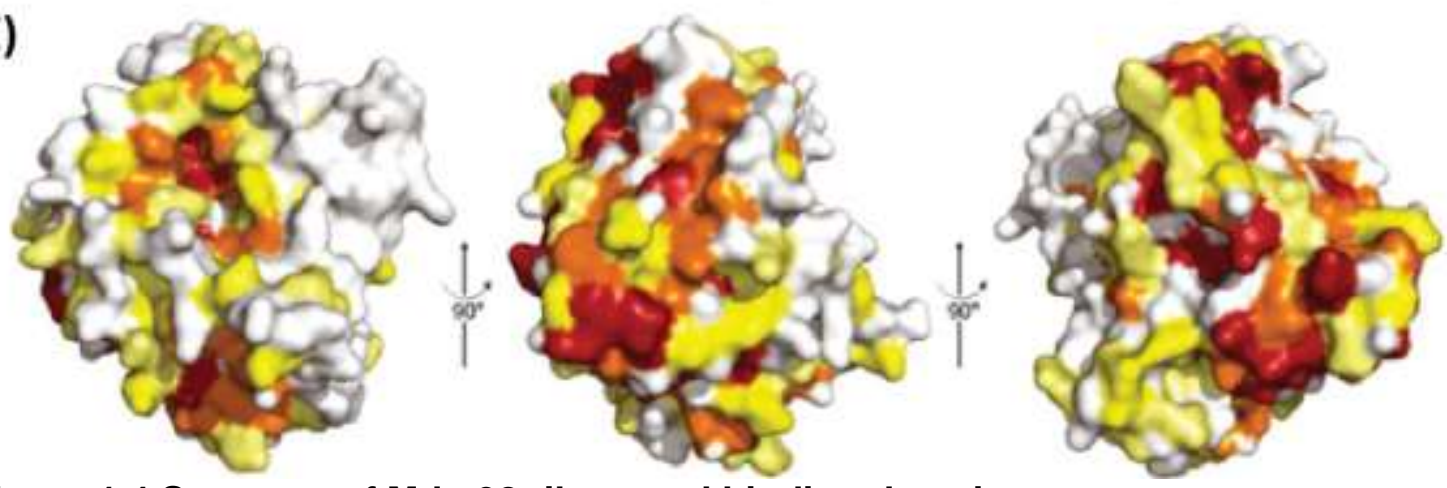

Figure 1.4 Structure of Mdm38 ribosomal binding domain

Shown are representations of the structure of Mdm38K in three different orientations after indicated rotations of about $90^{\circ}$ :

A) Ribbon models using rainbow colors from the $\mathrm{N}$-terminus (blue) to the $\mathrm{C}$-terminus (red).

B) Surface models of electrostatic charge distribution of Mdm38K. Negative charges are displayed in red, positive charges in blue and, uncharged in white.

C) Surface models showing the degree of amino acid conservation among Mdm38 protein family members determined via CONSURF analysis (Glaser et al. 2003). Highly conserved, conserved or partially conserved amino acids are displayed in dark red, orange and yellow, respectively.

Pictures taken from Lupo et al., 2011

Altogether, the results described above are in line with the model proposed by Bauerschmitt et al. (Figure 1.5): Mdm38 is localized in a membrane-associated context in the mitochondrial matrix. Furthermore, Mdm38 binds to mitochondrial ribosomes, which might indicate a similar role in positioning the ribosome as described for Mba1 (Ott et al. 2006; Keil et al. 2012). Finally, the interaction with 
translation factors suggests a recruitment of those proteins to the translation machinery, which in turn interact specifically with respective mRNA.

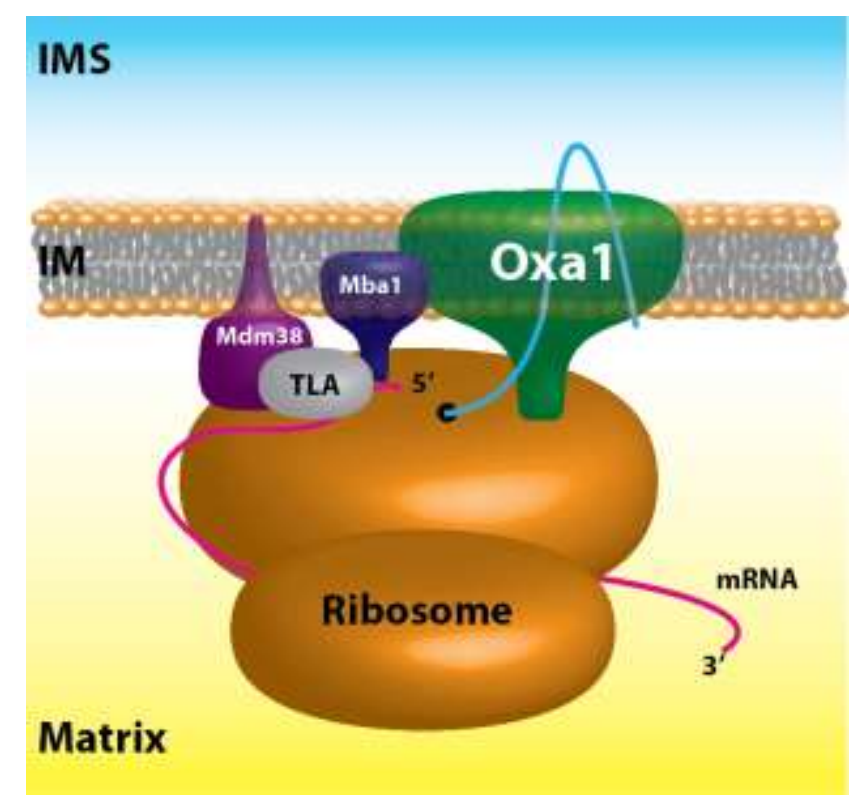

Figure 1.5 Model for the roles of Mdm38 and Mba1 in translation Illustrated are the ribosome-associated membrane proteins Oxa1, Mba1 and Mdm38. Translational activators (TLA) interact with Mba1 and Mdm38 and associate with the 5'-UTR of specific mRNAs (pink).

Adapted from

Bauerschmitt et al. 2010

\subsubsection{Ylh47 and Ydl183c are functionally related to Mdm38}

The functional variability of Mdm38 already suggests that the protein does not fulfill its different roles within mitochondria alone, but rather in complex with other proteins. This should be especially true for Mdm38 function in ion channeling.

First of all, Ylh47 (Yeast Letm1 homolog of $47 \mathrm{kDa}$ ) was investigated as a candidate for functional interactions, because it represents a homolog of Mdm38 in yeast. Although co-isolations indicate a physical interaction between Ylh47 and Mdm38, no function could be assigned to Ylh47, since no phenotype was observed for the respective deletion (Frazier et al. 2006). However, a genomic screen revealed Ylh47, together with an uncharacterized protein Ydl83c, to be multicopy suppressors of the $\Delta m d m 38$-phenotype (Zotova et al. 2010). Correspondingly, additional deletion of $Y D L 183 c$ and/or YLH47 enhanced the phenotypes of $\Delta m d m 38$. It was therefore concluded that Mdm38, Ylh47, and Ydl183c are functionally redundant, but Mdm38 has the highest impact.

Finally, it should also be noted that Zotova et al. (2010) revealed a selfdimerization of Mdm38 in mitochondria, of which the functional relevance remains unclear. 


\subsection{The Letm1-family}

Mdm38 is a member of a conserved, ubiquitously expressed, protein family present in all sequenced eukaryotes. The family is named after the human representative Letm1 (leucine zipper EF-hand containing transmembrane protein). As indicated by its name, the human $84 \mathrm{kDa}$ protein Letm 1 is predicted to contain a leucine zipper, two EF-hand $\mathrm{Ca}^{2+}$ binding domains, a transmembrane domain, and coiled-coil domains (Endele et al. 1999; Schlickum et al. 2004). The function of Letm 1 in higher eukaryotes is even more controversial than that of Mdm38 in yeast. Although complementation experiments in the MDM38 deletion mutant in yeast, using the human gene product (hLetm1) and Letm1 from Drosophila melanogaster (DmLetm1), indicate functional conservation, its role is still under debate.

On one hand, a genome-wide siRNA screen in drosophila identified DmLetm1 as a player in $\mathrm{Ca}^{2+}$-uptake (Jiang et al. 2009), a finding that was further supported by experiments using mouse, rat, and human cells in culture, as well as in hLetm1containing liposomes (Jiang et al. 2009; Waldeck-Weiermair et al. 2011; Tsai et al. 2014; Doonan et al. 2014). On the other hand, $\mathrm{K}^{+}$rather than $\mathrm{Ca}^{2+}$ was assumed to be the Letm1-specific ion. In support of this, the addition of the $\mathrm{K}^{+} / \mathrm{H}^{+}$ionophore nigericin rescued the mitochondrial fragmentation and matrix swelling phenotype of Letm1 depleted human cells, like in yeast (Nowikovsky et al. 2007; Hasegawa and van der Bliek 2007; Dimmer et al. 2007). Moreover, recent work in the protozoan flagellate, Trypanosoma brucei evansi, demonstrated a role for its Letm1 homologue in mitochondrial translation and potassium homeostasis, but not calcium homeostasis (Hashimi et al. 2013).

Interestingly, Letm 1 function in Arabidopsis thaliana seems to be even more enigmatic (Zhang et al. 2012). The plant genome encodes two Letm1 homologues, Atletm1 and Atletm2, with overlapping function and a synthetically lethal double deletion. Accordingly, hemizygous mutants are viable but show a generally reduced translation of mitochondrial genes. Furthermore, Western Blot and Blue Native analysis of these mutants demonstrates severe deficiencies in the ATP synthase. However, no morphologic phenotype and no change in protein levels of the respiratory chain complexes I, II, III and IV were detectable.

Altogether, these results for different organisms underline the complexity of roles 
in which Letm 1 proteins are involved. Therefore, contrary results regarding ionspecificity might indicate intertwining mechanisms for calcium and potassiumexchange, respectively.

\subsubsection{Letm 1 in the endoplasmic reticulum}

In contrast with previous publications that claim Letm 1 to be exclusively localized in mitochondria, fluorescence microscopy and Western blot analysis revealed the presence of hLetm 1 to be in the endoplasmic reticulum (ER) (Kuum et al. 2012). Correspondingly, the ER retention signal AEVK was identified in the C-terminus of hLetm1, in addition to its N-terminal mitochondrial targeting sequence. In their studies, Kuum and coworkers demonstrate that Letm1 has a role in ER pH homeostasis and is involved in $\mathrm{Ca}^{2+}$ uptake in specific cell types. Moreover, it was shown that $\mathrm{K}+/ \mathrm{H}+$-exchange is essential for $\mathrm{ER} \mathrm{Ca}^{2+}$ uptake using different inhibitors.

In summary, Kuum et al. nicely outline the dependence of calcium and potassiumexchange in the ER and indicate a function for hLetm 1 in this context. Therefore, both statements are in agreement with observations in mitochondria.

\subsection{Roles of Letm1 in human}

In line with the reported phenotypes on the cellular level, human Letm1 is also related to the Wolf-Hirschhorn syndrome (WHS), a multigenic disorder that affects 1 in 50,000 live births (Hirschhorn and COOPER 1961; Endele et al. 1999). The syndrome is defined by the association of atypical facial appearance, severe growth delay and, mental retardation with microcephaly. Additionally, WHS is associated with an impairment of muscular tone and seizures. (Endele et al. 1999). Thereby, observed symptoms can be correlated with the phenotypes in yeast, since dysfunction of mitochondrial oxidative phosphorylation is often related to neuronal and muscular degenerative diseases like WHS (DiMauro and Hirano 2009).

WHS is caused by partial deletion of chromosome 4 at locus $4 \mathrm{p} 16.3$, whereas the critical region (WHSCR-1) has been defined to a $165 \mathrm{~kb}$ region (Wright et al. 1997; Zollino et al. 1999). Unfortunately, this region contains multiple other genes, in addition to LETM1, resulting in a multifunctional pathology and thereby complicating a direct determination of genotype-phenotype correlations (Zollino 
et al. 2003). Deletions of hLETM1 occur in almost all patients with WHS (Endele et al. 1999), most of which exhibit seizures. Interestingly, the gene is preserved in those patients without seizures (Zollino et al. 2003; South et al. 2008).

Letm1 was also found to play a role in cancer diseases. In this context, the expression levels of hLetm 1 were shown to be significantly elevated in different human cancer tissues (Piao et al. 2009; Chen et al. 2014). Furthermore, studies showed that overexpression of hLetm 1 can induce necrotic cell death in HeLa cells, presumably due to depletion of intracellular ATP (Piao et al. 2009).

Interestingly, Hwang et al. (2010) showed that adenovirus-mediated overexpression of hLetm 1 could induce the destruction of mitochondria in lung cancer cells by depleting ATP and activating AMP-activated protein kinase (AMPK). Consequently, hLetm1 was suggested to function as a tumor suppressor gene for lung cancer (Hwang et al. 2010) .

If these effects are in fact directly or indirectly coupled to the functions of hLetm1 still remains unclear. However, a recent study reveals a link between hLetm1 knock down and the production of reactive oxygen species (ROS), which are one of the main causes of DNA-damage and thus, carcinogenesis (Doonan et al. 2014). 


\subsection{Research objectives}

The aim of these studies was to characterise the proteomic environment of Mdm38. Both in vitro and in vivo approaches were undertaken. For an in-vivo analysis, a fusion-construct with the biotin ligase BirA was introduced in the yeast Saccharomyces cerevisiae. This enabled the spatially restricted biotin tagging of proteins and thus, subsequent isolation and analysis.

In parallel, studies were performed with recombinant constructs of the ribosomal binding domain of Mdm38, expressed in Escherichia coli. In vitro binding to isolated E. coli ribosomes was demonstrated for two different constructs and further characterized with regard to the ionic environment.

Moreover, proteins in close proximity to $\mathrm{Mdm} 38$ where investigated in E. coli. An in vivo photo crosslinking approach was established using the non-natural amino acid para-benzoylphenylalanine (pBpA). A pBpA-specific cross-linking product was identified and analysed via mass spectrometry.

Finally, a novel phenotype caused by a mild overexpression of Mdm38, which was discovered within these studies, was further characterized. Therefore, different mitochondrial features related to functions of Mdm38 were addressed. 


\section{Materials and methods}

\subsection{Materials}

\subsubsection{Enzymes}

Digestion of DNA was performed with "FastDigest" restriction enzymes from Thermo Scientific (Waltham, Massachusetts). Additional enzymes used in this thesis are listed below.

Tab. 2.1 Enzymes and supplier

\begin{tabular}{|ll|}
\hline Enzyme & Supplier \\
Creatine Kinase & Roche (Basel) \\
Proteinase K & Roche (Basel) \\
TEV Protease & Invitrogen (Carlsbad, California) \\
Zymolyase 20T & Seikagaku Biobuiness (Fokushima) \\
\hline
\end{tabular}

\subsubsection{Chemicals}

All standard chemicals were purchased from AppliChem (Darmstadt), Merck (Darmstadt), Roth (Karlsruhe), SERVA (Heidelberg) or Sigma-Aldrich (Taufkirchen) in analytical grade purity. Exceptions and special chemicals are listed below.

Tab. 2.2 Special chemicals

\begin{tabular}{|ll|}
\hline Chemical & Supplier \\
Alexa Fluor $® 488$ C5 Maleimide & Thermo Fisher Scientific \\
ATti-HA, mouse monoclonal antibody & Roche \\
Coomassie brilliant blue G250 & Roche \\
Coomassie brilliant blue R250 & Serva \\
Creatine phosphate & Serva \\
$p$-bezoylphenylalanine & Roche \\
Desthiobiotin & Bachem \\
Digitonin & IBA \\
Dropout mixes (CSM-Trp/CSM-Ura) & Calbiochem \\
\hline
\end{tabular}




\begin{tabular}{|ll|}
\hline ECL Western Blotting detection reagent & GE-Healthcare \\
GeneRuler DNA Ladder Mix & Fermentas \\
Goat anti rabbit HRP & Dianova \\
Goat anti mouse HRP & Dianova \\
HisProbe ${ }^{\text {TM}}$-HRP Conjugate & Thermo Fisher Scientific \\
Herring sperm DNA & Promega \\
IgG standard (bovine) & Biorad \\
Leupetin & Roche \\
NADH & Roche \\
Nigericin sodium salt & Sigma \\
Ni-NTA agarose & Qiagen \\
Oligonucleotides & Metabion \\
Peroxidase anti-peroxidase, antibody (PAP) & Sigma \\
PEG-4000 & Applichem \\
Peptone, yeast extract, YNB w/o aa & BD \\
SDS marker broad range & Biorad \\
Unstained Protein Standard III & Serva \\
[35S]-methionine & Hartmann Analytics \\
\hline & \\
\hline
\end{tabular}

\subsubsection{Kit systems}

Kit systems listed below were used according to the manufacturer's specifications.

Tab. 2.3 Kit Systems and supplier

\begin{tabular}{|l|l|}
\hline Enzyme & Supplier \\
\hline KOD Hot Start DNA polymerase & Novagen \\
QuickChange $^{\circledR}$ Site-Directed Mutagenesis Kit & Stratagene/ Agilent \\
Rapid DNA Ligation Kit & Fermentas \\
Wizard ${ }^{\circledR}$ Plus SV Minipreps DNA Purification System & Promega \\
Wizard ${ }^{\circledR}$ SV Gel and PCR Clean-Up System & Promega \\
\hline
\end{tabular}

\subsubsection{Antibodies}

In general, polyclonal primary antibodies that were used in this study were raised in rabbit either against a C-terminal peptide or against the whole protein. Secondary antibodies against rabbit immunoglobulin, coupled to horseradish- 
peroxidase (HRP) for use in Western blot detection, goat anti rabbit HRP and goat anti rabbit IR680 were purchased from Dianova (Hamburg) and LI-COR (Bad Homburg), respectively.

For immune detection of protein A, peroxidase anti-peroxidase (PAP) synthesized in rabbit was purchased and signals were enhanced by secondary antibodies against rabbit immunoglobulin, coupled to HRP.

\subsubsection{PCR-Primers}

Deoxyoligonucleotides used as PCR-primers in this study either for molecular cloning or site directed mutagenesis were purchased in standard desalted quality from Metabion (Martinsried).

Tab. 2.4 Primers for molecular cloning

\begin{tabular}{|ll|}
\hline \multicolumn{1}{|c|}{ Name } & \multicolumn{1}{c|}{ Sequence } \\
CV199 & CACGAGCTCTGCTGGAGATTC \\
CV200 & GTGGGTACCTAGCCTTGTTTCTTTCTACGG \\
oMD 457 & Pho-AAGCTCATCGAGATCAGAAAAAG \\
oMD 458 & TAGCGGCCGCTTAGTTGTAAACGGGATCTGGAATG \\
oMD 459 & TAGCGGCCGCTTATTTTCGAACTGCGGGTGGCTCCA \\
oMD 467 & GGCTAGCCCATGGGCAAGCATCACCATCATTCAGGC \\
oMD 468 & TCCAAGCTCAGCTAATTAAGCTT \\
\hline
\end{tabular}


Tab. 2.5 Primers for site directed mutagenesis

\begin{tabular}{|c|c|c|}
\hline Name & AA-Exchange & $\begin{array}{l}\text { Sequence } \\
\text { (changed bases are written in small letters) }\end{array}$ \\
\hline oJMW_1 & C315 amber- stop & $\begin{array}{l}\text { AAGAAGAGCTGTATCAAGCTgCTGTGTCTagaGGTATGAAAGCGT } \\
\text { ACGGTGT }\end{array}$ \\
\hline oJMW_2 & C315 amber-stop & $\begin{array}{l}\text { ACACCGTACGCTTTCATACCtctAGACACAgCAGCTTGATACAGC } \\
\text { TCTTCTT }\end{array}$ \\
\hline oJMW_3 & C315A & $\begin{array}{l}\text { AAGAAGAGCTGTATCAAGCTTagGTGTCTagaGGTATGAAAGCGT } \\
\text { ACGGTGT }\end{array}$ \\
\hline oJMW_4 & C315A & $\begin{array}{l}\text { ACACCGTACGCTTTCATACCtctAGACACctAAGCTTGATACAGC } \\
\text { TCTTCTT }\end{array}$ \\
\hline oJMW_5 & G319A & TGTATCAAGCTTGTGTGTCTagaGcTATGAAAGCGTACGGTGTAT \\
\hline oJMW_6 & G319A & ATACACCGTACGCTTTCATAgCtctAGACACACAAGCTTGATACA \\
\hline oJMW_7 & M320A & CAAGCTTGTGTGTCTCGTGGcgCCAAAGCGTACGGTGTATCCAA \\
\hline oJMW_8 & M320A & TTGGATACACCGTACGCTTTggCgCCACGAGACACACAAGCTTG \\
\hline oJMW_9 & L330A & $\begin{array}{l}\text { ACGGTGTATCCAAGGAGGACgCgGTCGACAATCTAAAAGTTTGGC } \\
\text { TAGA }\end{array}$ \\
\hline oJMW_10 & L330A & $\begin{array}{l}\text { TCTAGCCAAACTTTTAGATTgTCGACCgCGTCCTCCTTGGATACA } \\
\text { CCGT }\end{array}$ \\
\hline oJMW_11 & W337A & GGTCGATAATCTAAAAGTTgсtCTAGAATTGAGACTGAGACA \\
\hline oJMW_12 & W337A & TGTCTCAGTCTCAATTCTAGagcAACTTTTAGATTATCGACC \\
\hline oJMW_13 & S354A & CATCCGTCTTGATGGTTTTGgctagCACGTTCACGTTTGGAGGAC \\
\hline oJMW_14 & S354A & GTCCTCCAAACGTGAACGTGctagcCAAAACCATCAAGACGGATG \\
\hline MVP83 & M320 amber-stop & CTTGTGTGTCTCGTGGTtagAAAGCGTACGGTGTAT \\
\hline MVP84 & M320 amber-stop & GATACACCGTACGCTTTCtaACCACGAGACACACAA \\
\hline MVP85 & S354 amber-stop & CATCCGTCTTGATGGTTTTtagTCCACGTTCACGTTTG \\
\hline MVP86 & S354 amber-stop & CTCCAAACGTGAACGTGGActaCAAAACCATCAAGACGGATG \\
\hline MVP87 & L330 amber-stop & CGGTGTATCCAAGGAGGACtagGTCGATAATCTAAAAGTTTG \\
\hline MVP88 & L330 amber-stop & CAAACTTTTAGATTATCGACctaGTCCTCCTTGGATACACCG \\
\hline MVP89 & G319 amber-stop & TATCAAGCTTGTGTGTCTCGTtagATGAAAGCGTACGGTGTATCC \\
\hline MVP90 & G319 amber-stop & GGATACACCGTACGCTTTCATCtaACGAGACACACAAGCTTGATA \\
\hline MVP91 & W337 amber-stop & GGTCGATAATCTAAAAGTTtagCTAGAATTGAGACTGAGAC \\
\hline MVP92 & W337 amber-stop & GTCTCAGTCTCAATTCTAGctaAACTTTTAGATTATCGACC \\
\hline
\end{tabular}




\subsubsection{Vectors}

Tab. 2.6 Plasmids for yeast and E. coli

\begin{tabular}{|c|c|c|c|}
\hline Plasmid & Backbone & Insert & Source \\
\hline pRS416 & & & Stratagene \\
\hline pRS416 Mdm38* & pRS416 & Mdm38 & This study \\
\hline YEp352 & & & (Hill et al. 1986) \\
\hline p9328-1 & YEp352 & Mdm38 & This study \\
\hline \multirow[t]{2}{*}{ pSUP } & & & (Ryu and Schultz \\
\hline & & & 2006) \\
\hline pGex4T2 & & & GE Healthcare \\
\hline pGex4T2 Mdm38K & pGex4T2 & GST-Mdm38 (aa182-408)-HIS6 & (Lupo et al. 2011) \\
\hline pGex4T3 & & & GE Healthcare \\
\hline pGex4T3 Mdm38cTD & pGex4T3 & GST-Mdm38 (aa159-573)-HIS6 & (Frazier et al. 2006) \\
\hline pBad24 & & & (Guzman et al. 1995) \\
\hline pBad24 Mdm38K* & pBad24 & HIS-SUMO-Mdm38(aa182-408)Strep & This study \\
\hline pTRc99a & & & Pharmacia Biotech \\
\hline pTRc99a Mdm38K* & pTRc99a & HIS-SUMO-Mdm38(aa182-408)Strep & This study \\
\hline pSUMO & & & AG Göhrlich \\
\hline pSUMO Mdm38K* & pSUMO & HIS-SUMO-Mdm38(aa182-408)Strep & This study \\
\hline
\end{tabular}

*additional derivatives of these vectors containing point mutations were used in this studies (Tab. 2.5)

\subsubsection{Laboratory Equipment}

\section{Tab. 2.7 Equipment}

\begin{tabular}{|l|l|l|}
\hline Product & Model & Supplier \\
Electrophoresis & EPS 601 power supply & GE Healthcare \\
& Hoefer SE600 Ruby Blue native system & GE Healthcare \\
& Mini-PROTEAN ${ }^{\circledR}$ 3 Cell & Bio-Rad \\
& Mini-Sub ${ }^{\circledR}$ Cell GT Cell & Bio-Rad \\
& PowerPac ${ }^{\text {TM }}$ HC Power Supply & Bio-Rad \\
& Semi Dry Blotting Chamber & PEQLAB \\
& & Biotechnologie \\
\hline Centrifuges & Sorvall RC 12BP & Thermo Scientific \\
& Sorvall ${ }^{\circledR}$ RC6 Plus & Thermo Scientific \\
& OptimaTMDX-XP & Beckman Coulter \\
& $5804 \mathrm{R}$ & Eppendorf \\
\hline
\end{tabular}




\begin{tabular}{|c|c|c|}
\hline & 12-MC & Beckman Coulter \\
\hline & $5415 \mathrm{R}$ & Eppendorf \\
\hline & $5417 \mathrm{R}$ & Eppendorf \\
\hline & 5424 & Eppendorf \\
\hline \multirow[t]{9}{*}{ Rotors } & Sorvall ${ }^{\circledR}$ F14S- $6 \times 250 Y$ & Thermo Scientific \\
\hline & Sorvall ${ }^{\circledR}$ F10S- $6 x 500 Y$ & Thermo Scientific \\
\hline & Sorvall ${ }^{\circledR}$ SS-34 & Thermo Scientific \\
\hline & Sorvall ${ }^{\circledR} \mathrm{H}-12000$ & Thermo Scientific \\
\hline & F45-24-11 & Eppendorf \\
\hline & F45-30-11 & Eppendorf \\
\hline & FA-45-24-11 & Eppendorf \\
\hline & TLA-55 & Beckman Coulter \\
\hline & $A-4-44$ & Eppendorf \\
\hline \multirow[t]{7}{*}{ Scanners } & Agfa Curix 60 Developing machine & Agfa \\
\hline & Autoradiography Storage Phosphor Screen & GE Healthcare \\
\hline & LAS 1000 & FujiFilm \\
\hline & Starion FLA-9000 & FujiFilm \\
\hline & Storm 820 Phosphorimager & GE Healthcare \\
\hline & Typhoon FLA 9500 Phosphorimager & GE Healthcare \\
\hline & UVsolo TS transilluminator & Biometra \\
\hline \multirow[t]{13}{*}{ Other } & Autoclave Systec DX-200 & Systec \\
\hline & Balance BP 3100P & Sartorius \\
\hline & Electronic Digital Balance Kern ABJ 220-4M & KERN Son \\
\hline & Excella ${ }^{\circledR}$ E10 platform shaker & New Brunswick \\
\hline & & Scientific \\
\hline & G25 Shaker Incubator & New Brunswick \\
\hline & & Scientific \\
\hline & Innova ${ }^{\circledR} 44$ Incubator shaker & New Brunswick \\
\hline & & Scientific \\
\hline & GeneQuant $^{\mathrm{TM}} 1300$ Spectrophotometer & GE Healthcare \\
\hline & Magnetic Stirrer MR 3001 & Heidolph \\
\hline & Milli-Q-Water purification system & Millipore \\
\hline & NanoVue ${ }^{\mathrm{TM}}$ Spectrophotometer & GE Healthcare \\
\hline
\end{tabular}




$\begin{array}{ll}\text { pH-meter } & \text { InoLab } \\ \text { Pipettes } & \text { Gilson } \\ \text { Sonicator Cell Disruptor (HI) } & \text { Heat Systems } \\ \text { Sonifier } 450 & \text { Branson } \\ \text { Storage phosphor screens } & \text { GE Healthcare } \\ \text { Potter S glass-Teflon Homogenizer } & \text { Sartorius AG } \\ \text { Termomixer Comfort } & \text { Eppendorf } \\ \text { TPersonal } 48 \text { thermocycler } & \text { Biometra } \\ \text { Vortex-Genie } 2 & \text { Scientific } \\ & \text { Industries }\end{array}$

\subsubsection{Cell lines}

Yeast (Saccharomyces cerevisiae - S. cerevisiae) strains used in this study were derivatives of YPH499 (Sikorski and Hieter 1989). Additional yeast strains transformed with different expression plasmids (Tab. 2.6) were used in this study are.

Escherichia coli (E.coli) strain XL1-blue (Stratagene) was used for molecular cloning. For protein expression E. coli strains C43 and BL21 were used.

Tab. 2.8 E. coli strains

\begin{tabular}{|lll|}
\hline Strain & Genotypes & Source \\
XL21-Blue & $\begin{array}{l}\text { supE44, hsdR17, recA1, endA1, gyrA96, thi-1, relA1, } \\
\left.\text { lac- }{ }^{\circ}(c), F^{\prime}[\text { proAB }+ \text {, lacIq lacZAM15, Tn10(tetr })\right]\end{array}$ & Stratagene \\
Bl21 & $\begin{array}{l}\text { F-ompT gal dcm lon hsdSB }(r B-m B-) \lambda(D E 3 ~[l a c I \\
\text { lacUV5-T7 gene } 1 \text { ind1 sam7 nin5]) }\end{array}$ & Novagen \\
C43 & $\begin{array}{l}\text { F-ompT gal hsd } S_{B}(r B-m B-) d c m \text { lon } \lambda D E 3 \text { and two } \\
\text { uncharacterized mutations described in the reference. }\end{array}$ & (Miroux alker 1996) \\
\hline
\end{tabular}


Tab. 2.9 S. cerevisiae strains

\begin{tabular}{|c|c|c|}
\hline Strain & Genotypes & Source \\
\hline YPH499* & $\begin{array}{l}\text { MATa, ade2-101 his3- } \Delta 200 \text { leu2- } \Delta 1 \text { ura3- } \\
52 \text { trp1- } \Delta 63 \text { lys2-801 }\end{array}$ & $\begin{array}{ll}\text { (Sikorski and } \\
\text { Hieter 1989) }\end{array}$ \\
\hline DaMY17 (mdm38s)* & $\begin{array}{l}\text { MATa ade2-1 his3-11,15 leu2-3,112 ura3-1 } \\
\text { trp1-1 mdm38::TRP1 }\end{array}$ & $\begin{array}{l}\text { (Bauerschmitt et } \\
\text { al. 2010) }\end{array}$ \\
\hline DaMY18 $(m d m 38 \Delta / m b a 1 \Delta)^{*}$ & $\begin{array}{l}\text { MATa ade2-1 his3-11,15 leu2-3,112 ura3-1 } \\
\text { trp1-1 mba1::HIS5MX6 mdm38::TRP1 }\end{array}$ & $\begin{array}{l}\text { (Bauerschmitt et } \\
\text { al. 2010) }\end{array}$ \\
\hline AFY25 (mdm38 ProtA $)^{*}$ & $\begin{array}{l}\text { MATa ade2-101 his3-_200 leu2_-_1 ura3-52 } \\
\text { trp1-_63 lys2-801 mdm38::MDM38ProtA- } \\
\text { HIS3MX6 }\end{array}$ & $\begin{array}{l}\text { (Frazier et al. } \\
2006)\end{array}$ \\
\hline AFY8 $(m b a 1 \Delta)$ & $\begin{array}{l}\text { MATa ade2-101 his3-_200 leu2___l ura3-52 } \\
\text { trp1-_63 lys2-801 mba1::HIS3MX6 }\end{array}$ & $\begin{array}{l}\text { (Bauerschmitt et } \\
\text { al. 2010) }\end{array}$ \\
\hline CVY1 (Mdm38 RBD-ProtA)* & $\begin{array}{l}\text { MATa, ade2-101 his3-200 leu2-1 ura3-52 } \\
\text { trp1-63 Lys2-801 mdm38::MDM38 (aa1- } \\
\text { 408) PROTA-HIS3MX6 }\end{array}$ & (Lupo et al. 2011) \\
\hline CVY1 (Mdm38 $\left.8^{\Delta \mathrm{RBD}-\mathrm{ProtA}}\right)^{*}$ & $\begin{array}{l}\text { MATa, ade2-101 his3-200 leu2-1 ura3-52 } \\
\text { trp1-63 Lys2-801 mdm38::MDM38 (aa1- } \\
\text { 159) PROTA-HIS3MX6 }\end{array}$ & (Lupo et al. 2011) \\
\hline $\cos 264$ & $\begin{array}{l}\text { MATa ade2-101 his3-_200 leu2-__l ura3-52 } \\
\text { trp1-_63 lys2-801 mdm38::MDM38- } \\
\text { HIS3MX6 }\end{array}$ & $\begin{array}{l}\text { Unpublished } \\
\text { Levchenko, } 2015\end{array}$ \\
\hline $\operatorname{cox} 26^{B i r A}$ & $\begin{array}{l}\text { MATa ade2-101 his3-_200 leu2-_l ura3-52 } \\
\text { trp1-_63 lys2-801 cox26::COX26BirA- } \\
\text { HIS3MX6 }\end{array}$ & $\begin{array}{l}\text { Unpublished } \\
\text { Levchenko, } 2015\end{array}$ \\
\hline$m d m 38^{\text {BirA }}$ & $\begin{array}{l}\text { MATa ade2-101 his3-_200 leu2_-_1 ura3-52 } \\
\text { trp1-_63 lys2-801 mdm38::MDM38BirA- } \\
\text { HIS3MX6 }\end{array}$ & $\begin{array}{l}\text { Unpublished } \\
\text { Levchenko, } 2015\end{array}$ \\
\hline
\end{tabular}

\subsection{Growth conditions and media}

\subsubsection{Cultivation of $S$. cerevisiae}

S. cerevisiae was grown in liquid cultures of rich media at $30^{\circ} \mathrm{C}$ with agitation. For selection of different markers, synthetic media lacking uracil and/or tryptophan were used. Media were composed as listed in (Tab. 2.1). Media and solutions and were either filter sterilized or autoclaved. To avoid maillard products, carbon sources were autoclaved separately and added later on. 
For solid media $2.5 \%$ agar was added before autoclaving and plates of $5 \mathrm{~mm}$ thickness were cast. Cultures from cryo-stocks were streaked out on solid media and stored at $30^{\circ} \mathrm{C}$ for 2-3 days. Liquid cultures were inoculated with cells picked from solid media or another liquid culture. Cell density of liquid cultures was monitored by $\mathrm{OD}_{600}$ whereby an $\mathrm{OD}_{600}$ of 1 approximately corresponds to $10^{7}$ cells/ml.

\section{Tab. 2.10 Yeast media}

\begin{tabular}{|l|l|}
\hline \multicolumn{1}{|c|}{ Component } & \multicolumn{1}{c|}{ Concentration } \\
YPD & 1\% yeast extract, 2\% peptone, 2\% glucose \\
YPGal & $1 \%$ yeast extract, $2 \%$ peptone, 2\% galactose \\
YPG & $1 \%$ yeast extract, $2 \%$ peptone, 3\% glycerol \\
SD-Ura & $0.67 \%$ yeast nitrogen base, 0.07\% CSM-Ura, 2\% glucose \\
SGal-Ura & $0.67 \%$ yeast nitrogen base, 0.07\% CSM-Ura, 2\% galactose \\
SG-Ura & $0.67 \%$ yeast nitrogen base, 0.07\% CSM-Ura, 3\% glycerol \\
SD-Trp & $0.67 \%$ yeast nitrogen base, 0.07\% CSM-Trp, 2\% glucose \\
SGal-Trp & $0.67 \%$ yeast nitrogen base, 0.07\% CSM-Trp, 2\% galactose \\
SG-Trp & $0.67 \%$ yeast nitrogen base, 0.07\% CSM-Trp, 3\% glycerol \\
SD-Trp/-Ura & $0.67 \%$ yeast nitrogen base, 1\% -Ura/-Trp-mix*, 2\% glucose \\
SGal-Trp/-Ura & $0.67 \%$ yeast nitrogen base, 1\% -Ura/-Trp-mix*, 2\% galactose \\
SG-Trp/-Ura & $0.67 \%$ yeast nitrogen base, 1\% -Ura/-Trp-mix*, 3\% glycerol \\
\hline *-Ura/-Trp-mix [w/v](filter sterilized): & $0.2 \%$ adenine, $0.2 \%$ histidine, 0.3\% leucine, 0,3 \% lysine
\end{tabular}

\subsubsection{Yeast cryo-stocks}

To store yeast strains at $-80^{\circ} \mathrm{C} 1 \mathrm{ml}$ liquid culture of approximately $1 \mathrm{OD}_{600}$ was supplemented with $15 \%$ sterile glycerol and frozen in liquid nitrogen.

\subsubsection{Drop dilution assay}

In order to monitor the growth of yeast strains, overnight cultures of approximately $2 \mathrm{OD}_{600}$ were harvested by centrifugation $\left(5 \mathrm{~min} / 5000 \mathrm{~g} / 4{ }^{\circ} \mathrm{C}\right)$ and washed with sterile water. Pellets were resuspended in water and adjusted to 2 $\mathrm{OD}_{600} .5 \mu \mathrm{l}$ of five serial 10-fold dilutions were spotted on solid medium applied with different carbon sources. After 2-5 days of growth at $30^{\circ} \mathrm{C}$ (optionally, also $24^{\circ} \mathrm{C} \& 37^{\circ} \mathrm{C}$ ) growth was documented by scanning (Epson Perfection V750 Pro). 
For growth on nigericin medium the drug was added to the solution (cooled down to $60^{\circ} \mathrm{C}$ ) in indicated concentrations prior to casting.

\subsubsection{Cultivation of $E$. coli}

E. coli cultures were grown at $37^{\circ} \mathrm{C}$ in LB medium (1\% yeast extract, $\left.0.5 \% \mathrm{NaCl}\right)$. Selective conditions for plasmids were achieved by the application of respective antibiotics. Therefore, $100 \mathrm{mg} / \mathrm{l}$ ampicillin sodium sulfate, $35 \mathrm{mg} / \mathrm{l}$ chloramphenicol or $30 \mathrm{mg} / \mathrm{l}$ kanamycin sulfate were used. For liquid cultures, E. coli cells were inoculated from solid media or from a pre-culture (1:100 to 1:1000). Cell density was monitored by optical density at $600 \mathrm{~nm}\left(\mathrm{OD}_{600}\right)$.

\subsubsection{E. coli cryo-stocks}

For stock preservation $1 \mathrm{ml}$ culture grown overnight was supplemented with $15 \%$ sterile glycerol, frozen in liquid nitrogen and stored at $-80^{\circ} \mathrm{C}$.

\subsection{Molecular biology methods}

\subsubsection{Isolation of yeast genomic DNA}

To extract genomic DNA from yeast, $5 \mathrm{ml}$ culture of wild-type yeast was grown to an $\mathrm{OD}_{600}$ of 0.5 to 0.7 . Subsequently the medium was removed by centrifugation ( $5 \mathrm{~min} / 5000 \mathrm{rpm}$ ) and cells were resuspended in $150 \mu \mathrm{l}$ resuspension buffer A and incubated for 1 hour at $37^{\circ} \mathrm{C}$. After the addition of $20 \mu \mathrm{l}$ of $10 \%$ SDS and 100 $\mu \mathrm{l} 8 \mathrm{M}$ ammonium acetate the suspension was incubated for $15 \mathrm{~min}$ at $-20^{\circ} \mathrm{C}$. Cell fragments were then separated from DNA by centrifugation (14.000 rpm/ $15 \mathrm{~min} /$ $4{ }^{\circ} \mathrm{C}$ ). The DNA containing supernatant was transferred to a new tube and DNA was precipitated by isopropanol that was added in equal volume. Finally DNA was pelleted by centrifugation (14.000 rpm/15 min/ $4^{\circ} \mathrm{C}$ ) and washed with $70 \%$ ethanol. After drying at RT, DNA was resuspended in $25 \mu \mathrm{l}$ TE buffer (1 mM EDTA, $10 \mathrm{mMTris} / \mathrm{HCl} \mathrm{pH} 7.5$ ) and stored at $-20^{\circ} \mathrm{C}$. 
Tab. 2.11 Resuspension Buffer A

\begin{tabular}{|l|l|}
\hline Component & Concentration \\
Tris/HCl pH 7.5 & $50 \mathrm{mM}$ \\
EDTA & $10 \mathrm{mM}$ \\
$\beta$-mercaptoethanol & $0.3 \%$ \\
Zymolyase & $0.5 \mathrm{mg} / \mathrm{ml}$ \\
\hline
\end{tabular}

\subsubsection{Determination of DNA concentration}

DNA concentration was determined via the absorption of DNA solutions. Therefore, $\mathrm{OD}_{260}$ was measured using NanoVue (GE Healthcare). According to (Sambrook et al. 1989) an $\mathrm{OD}_{260}$ of 1 was assumed to correspond to $50 \mu \mathrm{g} / \mathrm{ml}$ of double strand DNA.

\subsubsection{Sequencing of DNA}

Sequencing of DNA was performed by GATC Biotech (Konstanz). Obtained sequences were visualized and compared using GeniousPro (Version 5.3.6, Biomatters).

\subsubsection{Agarose gel electrophoresis}

DNA was separated by size with agarose gel electrophoresis. Therefore, 1-1.5 \% agarose was dissolved in TAE Buffer (2 mM EDTA, 40 mM Tris/acetate, pH 8.0) by heating it to near-boiling point in a microwave oven. After cooling down to $70{ }^{\circ} \mathrm{C}$ the solution was supplemented with $1 \mu \mathrm{g} / \mathrm{ml}$ ethidium bromide and casted while pockets were created with a comb. After complete gelling the gel was transferred to a Mini-Sub Cell GT chamber and covered with TAE Buffer. DNA samples in loading dye (10\% sucrose, $0.25 \%$ OrangeG) were pipetted into the pockets and the gel run was performed for $30 \mathrm{~min}$ at $120 \mathrm{~V}$. DNA bands were visualized on an UV-trans illuminator using GeneRuler DNA Ladder Mix (Fermentas) as a standard for size estimation. Optionally, DNA bands were cut out for purification on a UVlight table.

\subsubsection{Amplification of DNA fragments by PCR}

Polymerase chain reaction was performed to amplify DNA fragments using KOD Hot Start DNA polymerase (Novagen) according to the manufacturer's recommendation. In general, reactions were performed in $50 \mu \mathrm{l}$ using $10 \mathrm{ng}$ of 
plasmid DNA or 100 ng of yeast genomic DNA as template. Cycling conditions were adjusted for the intended fragment length and melting temperature of used primer pairs.

\subsubsection{Purification of plasmids}

Plasmids from E. coli were isolated using Wizard Plus SV Miniprep DNA Purification System (Promega) according to the manufacturer's specifications. Subsequently, nucleic acid concentration of the solution was determined and plasmids were stored in $\mathrm{dH} 2 \mathrm{O}$ at $-20^{\circ} \mathrm{C}$.

\subsubsection{Molecular cloning}

For cloning DNA into plasmid vectors the DNA of interest was amplified by PCR using primers with extensions that contain suitable restriction sites. Purified PCR products (Wizard ${ }^{\circledR}$ SV Gel and PCR Clean-Up System) and plasmids were cleaved with the same set of restriction enzymes and subsequently heat inactivated for 10 $\min$ at $85^{\circ} \mathrm{C}$. For dephosphorylation of the cut vector, the restriction solution was supplied with dephosphorylation buffer and alkaline phosphatase $(4 \mu \mathrm{l} / 1 \mu \mathrm{g}$ plasmid) and incubated for $45 \mathrm{~min}$ at room temperature. Afterwards, plasmids were separated from cut out fragments on a $1 \%$ agarose gel and isolated (Wizard ${ }^{\circledR}$ SV Gel and PCR Clean-Up System). Ligation was performed using the Rapid DNA Ligation Kit (Fermentas) according to the manufacturer's instructions. Ligated constructs were transformed into E. coli (XL1 blue). Finally, plasmids were isolated and constructs were confirmed by restriction analysis and sequencing.

\subsubsection{Site directed mutagenesis in plasmids}

Mutagenesis within plasmids was performed via mutagenesis-PCR using the Quickchange lightning kit (Agilent) according to the manufacturer's instructions. Therefore, primers containing intended nucleotide exchanges were designed. Achieved clones were finally examined by sequencing. Optionally, additional silent mutations were inserted into the primers by creating or removing low abundant restriction sites, which allow later verification of the mutagenesis by restriction analysis. 


\subsubsection{Enzymatic restriction of DNA}

Digestion of DNA with restriction enzymes was performed according to the manufacturer's recommendations (FastDigest-Thermo Scientific). Subsequently, enzymes were inactivated by $10 \mathrm{~min}$ incubation at $85^{\circ} \mathrm{C}$. DNA fragments were analyzed by agarose gel electrophoresis and used for ligation reactions.

\subsubsection{Transformation of yeast cells with lithium acetate}

Transformations of plasmid vectors into yeast were performed as described in (Gietz and Schiestl 2007). A $5 \mathrm{ml}$ overnight culture of the respective yeast strain was inoculated into $50 \mathrm{ml}$ YPD to $\mathrm{OD}_{600} 0.1$ and incubated shakingly at $30^{\circ} \mathrm{C}$. After reaching mid-log phase $\left(\mathrm{OD}_{600} 0.6\right.$ - 1.2) cells were centrifuged for $5 \mathrm{~min}(3.5 \mathrm{~g} /$ $4{ }^{\circ} \mathrm{C}$ ). The supernatant was discarded and the pellet washed twice with $50 \mathrm{ml}$ dH2O followed by a washing step with $25 \mathrm{ml} \mathrm{LiAc/TE}$ buffer. Subsequently, the resulting pellet was resuspended in $1 \mathrm{ml} \mathrm{LiAc/TE}$. For the transformation $100 \mu \mathrm{l}$ of cell suspension were mixed with 1-5 $\mathrm{g}$ plasmid and $10 \mu \mathrm{l}$ of carrier ssDNA (salmon sperm DNA $10 \mathrm{mg} / \mathrm{ml}$ ) which was previously denatured for $5 \mathrm{~min}$ at $95{ }^{\circ} \mathrm{C}$. After $30 \mathrm{~min}$ incubation at $30{ }^{\circ} \mathrm{C} 1 \mathrm{ml}$ of sterile filtered PEG4000 (40\% in LiAc/TE) were added and carefully mixed by pipetting up and down. The suspension was incubated for $30 \mathrm{~min}$ at $30{ }^{\circ} \mathrm{C}$ followed by an addition of $68 \mu \mathrm{l}$ DMSO and a heat shock of $42{ }^{\circ} \mathrm{C}$ for 15 min. Cells were then pelleted by two centrifugation steps of $5 \mathrm{~min}\left(5 \mathrm{~g} / 4^{\circ} \mathrm{C}\right)$ with turning the tubes $180^{\circ}$ in between. The supernatant was discarded and cells were resuspended in $100 \mu \mathrm{dH} 20$ for subsequent plating on respective selective agarose medium.

When colonies became visible after 2-3 d, three of them were streaked out again on selective media and single colonies were picked and tested for successful transformation.

\section{Tab. 2.12 LiAc/TE Buffer}

\begin{tabular}{|l|l|}
\hline Component & Concentration \\
LiAc, & $100 \mathrm{mM}$ \\
Tris/HCl (pH. 7.5) & $100 \mathrm{mM}$ \\
EDTA & $10 \mathrm{mM}$ \\
\hline
\end{tabular}




\subsubsection{Preparation of transformation competent $E$. coli cells with divalent cations}

$2 \mathrm{ml}$ of LB-medium was inoculated with a single colony of E. coli and incubated shakingly overnight at $37^{\circ} \mathrm{C}$. The whole culture was transferred to $200 \mathrm{ml} \mathrm{LB}$ medium and further incubated until an $0_{600}$ of 0.2-0.3 indicating the mid-log phase. After transferring the culture into $450 \mathrm{ml}$ falcon tubes it was stored on ice for $15 \mathrm{~min}$ and medium was discarded after centrifugation ( $5 \mathrm{~min} / 900 \mathrm{~g} / 4^{\circ} \mathrm{C}$ ). Cells were then gently resuspended in $67 \mathrm{ml}$ cold sterile filtered RF1-buffer and stored on ice for $30 \mathrm{~min}$. After $15 \mathrm{~min}$ centrifugation (5 min/ 900g/ $4{ }^{\circ} \mathrm{C}$ ) supernatant was removed and the pellet was resuspended in $16 \mathrm{ml}$ of cold sterile filtered RF2-buffer. After 15 min incubation on ice the cell suspension was split into aliquots of $10 \mu \mathrm{l}$ and snap frozen in liquid nitrogen. The achieved competent cells were stored frozen at $-80{ }^{\circ} \mathrm{C}$.

Tab. 2.13 RF1-buffer

\begin{tabular}{|ll|}
\hline Component & Concentration \\
$\mathrm{RbCl}$ & $100 \mathrm{mM}$ \\
$\mathrm{MnC}_{12}$ & $50 \mathrm{mM}$ \\
$\mathrm{Na}-$ acetate & $30 \mathrm{mM}$ \\
$\mathrm{CaCl}_{2}$ & $10 \mathrm{mM}$ \\
$\mathrm{Glycerol}$ & $15 \%(\mathrm{w} / \mathrm{v})$ \\
$\mathrm{RbCl}$ & $100 \mathrm{mM}$ \\
\hline Adjusted to pH 5.8 with $0.2 \mathrm{M}$ acetic acid \\
\hline
\end{tabular}

Tab. 2.14 RF2-buffer

\begin{tabular}{|ll|}
\hline Component & Concentration \\
$\mathrm{RbCl}$ & $10 \mathrm{mM}$ \\
$\mathrm{MnCl}_{2}$ & $50 \mathrm{mM}$ \\
$\mathrm{Na}-$ acetate & $10 \mathrm{mM}$ \\
$\mathrm{CaCl}_{2}$ & $75 \mathrm{mM}$ \\
$\mathrm{Glycerol}$ & $15 \%(\mathrm{w} / \mathrm{v})$ \\
$\mathrm{RbCl}$ & $10 \mathrm{mM}$ \\
\hline
\end{tabular}

Adjusted to $\mathrm{pH} 6.8$ with $50 \mathrm{mM} \mathrm{NaOH}$ 


\subsubsection{Transformation of chemically competent $E$. coli}

For transformation the competent cells were thawed on ice. Subsequently, $10 \mu \mathrm{l}$ ligation mix or $200 \mathrm{ng}$ plasmid DNA were added and the suspension was incubated for $15 \mathrm{~min}$ on ice followed by a heat shock for $45 \mathrm{~s}$ at $42{ }^{\circ} \mathrm{C}$. For establishing the resistance against respective antibiotics, cells were cooled down, transferred to $1 \mathrm{ml} \mathrm{LB}$ medium and incubated shakingly for $1 \mathrm{~h}$ at $37^{\circ} \mathrm{C}$ with. Afterwards, cells were pelleted by 5 min centrifugation $(5 \mathrm{~g})$. The supernatant was discarded and cells were resuspended in $100 \mu \mathrm{d}_{2} \mathrm{O}$ for subsequent plating on the respective selective agarose medium.

\subsection{Biochemical methods}

\subsubsection{Separation of proteins by SDS-PAGE}

Separation of proteins under denaturing conditions was performed via with SDSpolyacrylamide gel electrophoresis (SDS-PAGE). Prior the electrophoresis the proteins are boiled for $5 \mathrm{~min}$ at $95^{\circ} \mathrm{C}$ or kept at room temperature for $30 \mathrm{~min}$ in SDS sample buffer. Thus, SDS coats the proteins resulting in a negative overall charge, so that proteins move to the anode in an applied electric field. Thereby, higher weight proteins migrate slower through the pores of the gel while smaller proteins are faster. The low percentage stacking gel contains large pores and the proteins are forming a stack between the leading chloride ions and the trailing ion Glycine. Subsequently, proteins reach the resolving gel with a higher $\mathrm{pH}$ than the stacking gel and containing smaller pores. Thus, the proteins are separated according to their molecular weight. Depending on the molecular weight of the investigated proteins, the different percentages of acrylamide/ bisacrylamide are used within the resolving gel. Details on the composition of the gels can be found in the following overview:

For pouring and running SDS-gels self-made devices were used for large gels (gel size: $16.5 \mathrm{~cm} \times 14 \mathrm{~cm} \mathrm{X} \mathrm{1mm)} \mathrm{and} \mathrm{the} \mathrm{Mini-PROTEAN} \mathrm{II} \mathrm{Bio-Rad} \mathrm{system} \mathrm{for}$ smaller ones (gel size: $10 \mathrm{~cm}$ x $5.5 \mathrm{~cm}$ x 0.75). Therefore, two glass plates, which were separated by two spacers, were fixed together by metal clamps and positioned in a gel basis. The lower rim of the casting mold was sealed with $3 \mathrm{ml} 15$ $\%$ resolving gel supplied with $0.15 \%$ APS and $0.3 \%$ TEMED. After polymerization 
of the sealing gel the resolving gel is casted on top of it filling three quarters of the space between the glass plates. The gel was topped with isopropanol during the polymerization to create a plane surface and prevent dehydration. The polymerized gel was rinsed with water and the stacking gel was filled on top. To form separated pockets for the application of the protein samples, a comb with 1421 teeth was fit in the liquid gel.

The electrophoresis was performed in SDS running buffer (25 mM Tris, $1.91 \mathrm{M}$ glycine, $1 \%$ SDS) at $30 \mathrm{~mA}$ per gel using $250 \mathrm{~V}$.

Tab. 2.15 SDS-Polyacrylamide

\begin{tabular}{|c|c|c|}
\hline Component & $\begin{array}{l}\text { Concentration } \\
\text { (resolving gel) }\end{array}$ & $\begin{array}{l}\text { Concentration } \\
\text { (stacking gel) }\end{array}$ \\
\hline Acrylamide/Bisacrylamide (30/0.8 \%) & $10-16 \%$ & $5 \%$ \\
\hline Tris/HCl pH ph 8.8 & $380 \mathrm{mM}$ & - \\
\hline Tris/HCl pH ph 6.8 & - & $80 \mathrm{mM}$ \\
\hline SDS & $0.1 \%(\mathrm{w} / \mathrm{v})$ & $0.1 \%(\mathrm{w} / \mathrm{v})$ \\
\hline APS* & $0.06 \%(\mathrm{w} / \mathrm{v})$ & $0.1 \%(\mathrm{w} / \mathrm{v})$ \\
\hline TEMED* & $0.12 \%$ & $0.2 \%$ \\
\hline
\end{tabular}

* Added immediately before casting

Tab. 2.16 SDS Sample Buffer

\begin{tabular}{|l|l|}
\hline Component & Concentration \\
SDS & $2 \%(\mathrm{w} / \mathrm{v})$ \\
Glycerol & $10 \%$ \\
Tris/HCl pH 6.8 & $60 \mathrm{mM}$ \\
$\beta$-mercaptoethanol & $0.5 \%$ \\
Bromphenolblue & $0.01 \%(\mathrm{w} / \mathrm{v})$ \\
\hline
\end{tabular}

\subsubsection{Blue Native Page}

Blue Native Page (BN Page) according to (Schägger and Jagow 1991) was used to separate native protein complexes from either whole solubilized mitochondria or already purified protein samples. For latter ones 10x BN sample buffer is directly added. Whole mitochondria were first pelleted (10 min, $\left.16.000 \mathrm{~g}, 4{ }^{\circ} \mathrm{C}\right)$ and subsequently solubilized in Digitonin Buffer by pipetting up and down 20 times 
yielding to a protein concentration of $1 \mathrm{mg} / \mathrm{ml}$. The suspension was stored on a rotating wheel at $4{ }^{\circ} \mathrm{C}$ for $30 \mathrm{~min}$. After $10 \mathrm{~min}$ centrifugation $\left(16.000 \mathrm{~g} / 4^{\circ} \mathrm{C}\right)$ the supernatant was used for the assay. Therefore, 10x BN sample buffer was added to the sample.

Gels with different acrylamide gradients (Acrylamide/ Bisacrylamide: 16/1) were cast directly before the gel run and cooled down to $4{ }^{\circ} \mathrm{C}$. Samples were loaded on the gel and covered with precooled BN Cathode Buffer $(15 \mathrm{mM}$ Bis-Tris/HCl pH 7.0, 50 mM Tricine, 0.2\% Coomassie Brilliant Blue G-250) completely filling up the gel pockets. The gel electrophoresis was performed in Anode Buffer (50 mM Bis-Tris/HCl pH 7.0) using the SE600 Ruby system (GE-Healthcare) at a $200 \mathrm{~V}$ and $15 \mathrm{~mA}$. In the middle of the gel run the cathode buffer was replaced with BN Cathode Buffer without coomassie. Electrophoresis was continued either at $600 \mathrm{~V}$ for 4-5 h or overnight at $100 \mathrm{~V}$.

Tab. 2.17 10x BN Sample Buffer

\begin{tabular}{|ll|}
\hline Component & Concentration \\
\hline Bis- Tris/HCl, (pH 7.0) & $100 \mathrm{mM}$ \\
$\boldsymbol{\varepsilon}$-Amino n-caproic acid & $500 \mu \mathrm{M}$ \\
Coomassie Brilliant Blue G-250 & $5 \%$ \\
\hline
\end{tabular}

Tab. 2.18 Digitonin Solubilization Buffer

\begin{tabular}{|ll|}
\hline Component & Concentration \\
\hline Tris/HCl pH 7.4 & $20 \mathrm{mM}$ \\
$\mathrm{NaCl}$ & $50 \mathrm{mM}$ \\
Glycerol & $10 \%$ \\
EDTA & $1 \mathrm{mM}$ \\
Digitonin & $1 \%$ \\
PMSF & $2 \mathrm{mM}$ \\
\hline
\end{tabular}

\subsubsection{Western blotting of polyacrylamide gels}

For further analyses by immunodetection, proteins separated on polyacrylamide gels were transferred to PVDF membranes via using semi dry Western blotting. Therefore, gel and membrane activated in methanol were equilibrated in Blotting Buffer (20 mM Tris, $150 \mathrm{mM}$ glycine, 0.02\% SDS, $20 \%$ ethanol). The blot was 
assembled in a blotting chamber (Peqlab) using 3 layers of Whatman paper soaked with Blotting Buffer followed by the membrane the gel and another 3 layers of Whatman paper. The blotting run was performed at $250 \mathrm{~mA} / 25 \mathrm{~V}$ for $2.5 \mathrm{~h}$. Subsequently, membranes were directly used for further analysis or dried on a Whatman paper and stored at $4{ }^{\circ} \mathrm{C}$.

\subsubsection{Immunostaining of Western Blot membranes}

PVDF membranes were blocked in TBST buffer (125 mM NaCl, 0.1\% Tween 20, $20 \mathrm{mM}$ Tris, $\mathrm{pH}$ 7.5) supplied with $5 \%$ low fat milk powder for $1 \mathrm{~h}$ at room temperature or at $4{ }^{\circ} \mathrm{C}$ overnight. Membranes were incubated in primary antibody for $1 \mathrm{~h}$ at room temperature and subsequently washed 3 times for $10 \mathrm{~min}$ in TBST followed by another incubation of $1 \mathrm{~h}$ at room temperature with the respective secondary antibody coupled to HRP. After 3 washing steps of $10 \mathrm{~min}$ in TBST membranes were placed in a developing cassette and supplied with ECL solution. Signals were detected using x-ray films.

\subsubsection{Western Blot staining with tag-specific probes}

To detect biotinylated or histidine tagged proteins on Western Blots, HRP-coupled probes were used (His: HisProbe ${ }^{\text {TM}}$-HRP (Thermo Fisher) / Biotin: HRP-conjugated streptavidin (SA-HRP - Invitrogen)). Activated PVDF membranes were therefore blocked in TBST buffer (2.4.5) containing $3 \%$ BSA for $1 \mathrm{~h}$ at room temperature or at $4{ }^{\circ} \mathrm{C}$ overnight. After washing in TBST membranes were incubated with probes diluted 1:1000 in TBST for $1 \mathrm{~h}$ at room temperature or at $4{ }^{\circ} \mathrm{C}$ overnight. After final washing steps ( 3 x 10 min in TBST) signal detection was performed as in 2.4.5.

\subsubsection{Coomassie staining of Western Blot membranes or polyacrylamide gels}

To visualize proteins on PVDF membranes or polyacrylamide gels subjects were incubated in Coomassie staining solution $(2.5 \mathrm{~g} / \mathrm{l}$ Coomassie brilliant blue R-250, $40 \%$ ethanol, $10 \%$ acetic acid) for 5 min (PVDF membranes) or 3 hours (gels). Subjects were then transferred to destaining solution (40\% ethanol, $10 \%$ acetic acid) and shaken at room temperature until background staining was removed.

Before using PVDF membranes for immune staining they were completely destained in methanol. 


\subsubsection{Colloidal coomassie staining of polyacrylamide gels}

Colloidal coomassie staining was used to visualize protein bands of lower abundance on polyacrylamide gels according to (Neuhoff et al. 1985). Therefore, after gel run gels were directly transferred to fixing solution (40 \% EtOH, $10 \%$ Acetic Acid) so that it was completely covered. After shaking incubation for at least $1 \mathrm{~h}$ gels were washed twice for $10 \mathrm{~min}$ with water and subsequently transferred to freshly prepared dye solution (80 \% dye stock solution / $20 \%$ methanol (v/v)). Staining was performed for 1-3 days while shaking at room temperature.

\subsubsection{Preparation of colloidal Coomassie stock solution}

The dye stock solution was prepared at least $24 \mathrm{~h}$ in advance of the staining and for later uses. $2 \%(\mathrm{w} / \mathrm{v}$ ) ortho-phosphoric acid and $10 \%$ ammonium sulfate were dissolved in $80 \%$ of the final volume water. After complete dissolution $0.1 \%$ Coomassie Brilliant Blue G250 was added while stirring and the solution was filled up to final volume with water. The stock solution was stored at room temperature but stirred again for at least $24 \mathrm{~h}$ before use.

\subsubsection{Protein extraction from whole yeast cells}

In order to perform steady state analysis of yeast proteins an extraction from whole cells was performed. Yeast strains were grown in overnight culture of $5 \mathrm{ml}$ to an $\mathrm{OD}_{600}$ less than 3. Cells of $2 \mathrm{OD}_{600}$ were then harvested by centrifugation at $5.000 \mathrm{~g}$ and subsequently resuspended in $1 \mathrm{ml}$ water. Cell lysis was performed by the addition of $15 \mathrm{mM} \beta$-mercaptoethanol and $250 \mathrm{mM} \mathrm{NaOH}$. After $10 \mathrm{~min}$ incubation on ice proteins were precipitated by the addition of $6 \%(\mathrm{w} / \mathrm{v})$ TCA followed by10 min incubation on ice. Proteins were pelleted by centrifugation (10 $\mathrm{min} / 14.000 \mathrm{~g}$ ), resuspended in $50 \mu \mathrm{l}$ sample buffer and boiled for $5 \mathrm{~min}$ at $95^{\circ} \mathrm{C}$.

\subsubsection{Determination of protein concentrations via Bradford assay}

Protein concentrations were determined via Bradford assay using Roti-Quant (Roth) according to the manufacturer's recommendations. Therefore, the absorbance of protein solutions was measured at $600 \mathrm{~nm}$. Concentrations were calculated using a standard curve prepared with IgG or BSA. 


\subsubsection{Subcellular fractionation of $S$. cerevisiae}

Subcellular fractionation was performed to isolate mitochondria from yeast cells. Therefore, a $50 \mathrm{ml}$ overnight culture under respiration enforcing conditions e.g. in YPG was inoculated in 200-500 $\mathrm{ml}$ of respective media. The next day main cultures of $1.8 \mathrm{~L}$ were inoculated and adjusted to an $\mathrm{OD}_{600}$ resulting in $\mathrm{OD}_{600}$ of 2 after another overnight incubation. Main cultures were harvested by $10 \mathrm{~min}$ centrifugation (4.000 rpm / $18^{\circ} \mathrm{C}$ ). Cells were resuspended in $500 \mathrm{ml}$ water and subsequently transferred to a $500 \mathrm{ml}$ centrifuge beaker. After $10 \mathrm{~min}$ centrifugation (4.000 rpm / $18^{\circ} \mathrm{C}$ ) the supernatant was discarded and the cell pellet was weighted. Cells were then resuspended in $2 \mathrm{ml}$ DTT-buffer/ mg cells (100 mM Tris/HCl pH 9.4, 10 mM DTT). For sufficient disruption of disulfide bonds within the cell wall, cells were incubated for $30 \mathrm{~min}$ at $30^{\circ} \mathrm{C}$ shaking with $220 \mathrm{rpm}$. DTT buffer was removed by two centrifugation steps $\left(4.000 \mathrm{rpm} / 18^{\circ} \mathrm{C}\right)$ including a resuspension in 1.2 M Sorbitol for further washing. Cells were then resuspended in $7 \mathrm{ml} / \mathrm{g}$ yeast zymolyase buffer $(20 \mathrm{mM}$ potassium phosphate $\mathrm{pH} 7.4,1.2 \mathrm{M}$ sorbitol) without enzyme. Per gram cells $4 \mathrm{mg}$ of zymolyase were dissolved in $1 \mathrm{ml}$ $1 \mathrm{M}$ Tris/HCl (pH 7.4) and added to the suspension. After 90 min shaking with $90 \mathrm{rpm}$ at $30^{\circ} \mathrm{C}$ suspensions were checked for spheroblasts microscopically, pelleted (3.000 rpm / $18{ }^{\circ} \mathrm{C}$ ) and washed with $100 \mathrm{ml}$ zymolyase buffer without enzyme. From now on all steps were performed on ice. Subsequent to the addition of PMSF to a final concentration of $1 \mathrm{mM}$ suspensions were transferred to a precooled $60 \mathrm{ml}$ glass-Teflon homogenizer (Potter S, Sartorius). For homogenization by mechanic disruption of the cell membrane the Teflon pistil was moved up and down 20 times rotating with $900 \mathrm{rpm}$. Suspensions were then cleared from higher weight cell fragments by two subsequent centrifugation steps $\left(10 \mathrm{~min} / 7000 \mathrm{~g} / 4{ }^{\circ} \mathrm{C}\right)$ followed by a centrifugation $\left(15 \mathrm{~min} / 17000 \mathrm{~g} / 4^{\circ} \mathrm{C}\right)$ of the supernatant to pellet mitochondria. Afterwards, Mitochondria were washed with 5 ml SEM buffer (1 mM PMSF) and subsequently resuspended in a final volume of 1 ml SEM buffer. Finally, protein concentration was determined with Bradford Assay and adjusted to $10 \mu \mathrm{g} / \mu \mathrm{l}$ by addition of SEM buffer. Mitochondria were frozen in liquid nitrogen and stored at $-80^{\circ} \mathrm{C}$. 
Tab. 2.19 Homogenization buffer

\begin{tabular}{|l|l|}
\hline Component & Concentration \\
Sorbitol & $1.2 \mathrm{M}$ \\
Tris pH 7.4 & $20 \mathrm{mM}$ \\
EDTA & $2 \mathrm{mM}$ \\
BSA (fatty acid free) & $0.4 \%(\mathrm{w} / \mathrm{v})$ \\
\hline
\end{tabular}

\subsubsection{In vivo labeling of mitochondrial translation products}

In order to analyze mitochondrial translation products quantitatively in living yeast cells, radioactive in vivo labeling via ${ }^{35} \mathrm{~S}$-methionine was performed. For that reason yeast cells were grown in $5 \mathrm{ml}$ medium (2.2.1) overnight using galactose as the carbon source. The next day solutions were diluted to $\mathrm{OD}_{600}$ of 0.1 . After reaching an $\mathrm{OD}_{600}$ of 1 cells were harvested by centrifugation ( $1 \mathrm{~min} / 5000 \mathrm{~g} / \mathrm{RT}$ ) and washed by centrifugation ( $1 \mathrm{~min} / 5000 \mathrm{~g} / \mathrm{RT}$ ) with $500 \mu$ labeling buffer (2\% Gal, $40 \mathrm{mM} \mathrm{KPi} \mathrm{pH} \mathrm{6.0).} \mathrm{Pellets} \mathrm{were} \mathrm{resuspended} \mathrm{in} 500 \mu \mathrm{l}$ labeling buffer and incubated shakingly at $30{ }^{\circ} \mathrm{C}$ for methionine starvation. To inhibit cytosolic translation cyclohexemid was added to a final concentration of $0.5 \mathrm{mM}$. After further $5 \mathrm{~min}$ incubation at $30^{\circ} \mathrm{C}$. Mitochondrial translation was started by the addition of $2 \mu{ }^{35} \mathrm{~S}$-methionine $(10 \mathrm{mCi} / \mathrm{ml})$ and suspensions were shaken at $30{ }^{\circ} \mathrm{C}$. After a defined period of time (pulse) further integration of $35 \mathrm{~S}$-methionine is prevented by the addition of an excess of methionine ( $4 \mathrm{mM})$. After $2 \mathrm{~min}$ of further incubation suspensions were put on ice.

After a following whole cell extraction the achieved protein pellets were resuspended in $50 \mu \mathrm{l}$ SDS-sample buffer and kept shaking at room temperature for $30 \mathrm{~min}$. For further analysis, samples were subjected to SDS-PAGE (16\% acrylamide/ $0.21 \%$ bisacrylamide) and digital autoradiography, which was optionally combined with Western Blot analysis.

\subsubsection{Analysis of stability of mitochondrial translation products in vivo with pulse-chase experiments}

Optionally, the stability of newly synthetized mitochondrially encoded proteins was investigated by in vivo labeling within pulse-chase experiments. Therefore, cells were further shaken at $30{ }^{\circ} \mathrm{C}$ after the addition of non-labeled methionine for defined time frames (chase), subsequently put on ice and treated as described 
above.

\subsubsection{In organello labeling of mitochondrial translation products}

Translation products from intact isolated mitochondria were analyzed quantitatively by radioactive in organello labeling with ${ }^{35} \mathrm{~S}$-methionine. Therefore, $40 \mu \mathrm{g}$ mitochondria were thawed on ice and carefully suspended in $40 \mu \mathrm{l}$ freshly prepared $1.5 \mathrm{x}$ translation buffer. Thereupon, pyruvate was added to a final concentration of $12 \mu \mathrm{g} / \mathrm{ml}$ and the solution was filled up to $60 \mu \mathrm{l}$ with water. After 5 min preincubation at $30^{\circ} \mathrm{C}$ mitochondrial translation was started by the addition of $2 \mu{ }^{35} \mathrm{~S}$-methionine $(10 \mathrm{mCi} / \mathrm{ml})$ and suspensions were shaken at $30^{\circ} \mathrm{C}$. After a defined pulse an excess of unlabeled methionine $(4 \mathrm{mM})$ was added to prevent further incorporation of ${ }^{35} \mathrm{~S}$-methionine. After 2 min further incubation at $30{ }^{\circ} \mathrm{C}$ samples were put on ice. Mitochondria were then isolated by centrifugation (10 min/ 14,000 g/ $\left.4^{\circ} \mathrm{C}\right)$ and washed with SEM. Pellets were resuspended in SDS sample buffer and shaken for $30 \mathrm{~min}$ at RT. Samples were further analyzed via SDS-PAGE (16\% acrylamide/ $0.21 \%$ bisacrylamide) with and digital autoradiography and optionally also with Western Blot analysis.

\section{Tab. 2.20 1.5x Translation buffer}

\begin{tabular}{|ll|}
\hline Component & Concentration \\
Sorbitol & $900 \mathrm{mM}$ \\
K-phosphate pH 7.4 & $225 \mathrm{mM}$ \\
Tris pH 7.4 & $22.5 \mathrm{mM}$ \\
BSA & $30 \mathrm{mM}$ \\
ATP pH 7.2 & $4.5 \mathrm{mg} / \mathrm{ml}$ \\
GTP pH 7.2 & $6 \mathrm{mM}$ \\
2-Ketoglutarat & $0.75 \mathrm{mM}$ \\
Creatin Phosphate & $9 \mathrm{mM}$ \\
AA-mix (-met) & $7.5 \mathrm{mM}$ \\
CHX (in EtOH) & $0.15 \mathrm{mM}$ \\
MgSO4 & $7.5 \mu \mathrm{ml}$ \\
\hline
\end{tabular}




\subsubsection{Stability analysis of mitochondrial translation products in organello via within pulse-chase experiments}

In addition to quantitative analysis of translation over time also the stability of mitochondrial encoded proteins can be investigated in this context via using pulsechase experiments. Therefore, labeling was performed as described above for a pulse of $10 \mathrm{~min}$ followed by the addition of unlabeled methionine ( $4 \mathrm{mM})$. Stability was then observed for different time frames (chase) in samples which were shaken at $30{ }^{\circ} \mathrm{C}$ before they were put on ice. Afterwards samples were collected, washed and analyzed as described above.

\subsubsection{Submitochondrial localization of proteins by protease protection}

Localization of proteins in mitochondria was investigated by determining accessibility to proteinase $\mathrm{K}$ according to (Frazier et al. 2006). The assay was performed either in intact mitochondria or in mitoplasts using samples with completely disrupted membranes as a control. Mitoplasts were obtained by osmotic swelling. Therefore, mitochondria were diluted to a concentration of 1 $\mathrm{mg} / \mathrm{ml}$ in EM buffer and incubated on ice for $25 \mathrm{~min}$. Samples for intact mitochondria were kept in SEM buffer with the same concentration and also stored on ice during that time. Samples were then treated with $20-100 \mu \mathrm{g} / \mathrm{ml}$ proteinase $\mathrm{K}$ or left untreated followed by an incubation on ice for $10 \mathrm{~min}$. Blocking of the proteinase was performed by the addition of $2 \mathrm{mM}$ PMSF and further incubation of $10 \mathrm{~min}$ on ice. Control samples with completely disrupted membranes were prepared from mitochondria (1 $\mathrm{mg} / \mathrm{ml}$ in EM buffer) that were either treated with $100 \mu \mathrm{g} / \mathrm{ml}$ proteinase $\mathrm{K}$ or left untreated and subsequently exposed to $3 \mathrm{x} 20$ pulses in the ultrasound bath. After 10 min incubation on ice proteins were blocked as shown above. All samples were TCA precipitated and resuspended in $1 \mathrm{x}$ SDS sample buffer containing $1 \mathrm{mM}$ PMSF. All samples were separated via by SDSPAGE and the corresponding Western Blot was probed by antibodies for the protein of interest and also control proteins with known submitochondrial localization.

\subsubsection{Isolation of protein complexes using Protein A-tag}

To investigate the interactome of $\mathrm{Mdm} 38$, mitochondria from yeast strains expressing Mdm38 chromosomally tagged with Protein A were used. Therefore, 
mitochondria were thawed on ice and spun down by centrifugation (10 min/ $14,000 \mathrm{~g} / 4^{\circ} \mathrm{C}$ ). Pellets were resuspended in digitonin solubilization buffer creating a final concentration $1 \mathrm{mg} / \mathrm{ml}$ protein. After $30 \mathrm{~min}$ incubation on an end-over-end shaker at $4{ }^{\circ} \mathrm{C}$, solutions were cleared by centrifugation $\left(15 \mathrm{~min} / 14,000 \mathrm{~g} / 4^{\circ} \mathrm{C}\right.$ ). Supernatants were then subjected to activated and equilibrated IgG-Sepharose beads (see 2.4.18, $50 \mathrm{ml}$ beads per $1 \mathrm{mg}$ mitochondria). After $2 \mathrm{~h}$ rotation on the end-over-end shaker at $4{ }^{\circ} \mathrm{C}$ beads were cleared from unbound material by centrifugation ( $30 \mathrm{~s} / 100 \mathrm{~g} / 4^{\circ} \mathrm{C}$ ) and 10 washing steps using 10 excess of washing buffer. Native elution from the beads was performed by enzymatic cleavage of the Protein A tag with TEV-protease. Therefore, beads were shaken overnight (800 $\mathrm{rpm} / 4^{\circ} \mathrm{C}$ ) in a $1: 1$ suspension with washing buffer supplied with $1 \mu \mathrm{l}$ TEVprotease per $100 \mu \mathrm{l}$. TEV-protease was subsequently removed via its HIS-tag by NiNTA isolation. For that reason suspensions were supplied with $20 \%$ (v/v) Ni-NTA beads equilibrated with washing buffer ( $2 \mathrm{x}$ washing with $10 \mathrm{x}$ excess). Elutions were then isolated by centrifugation $\left(30 \mathrm{~s} / 100 \mathrm{~g} / 4^{\circ} \mathrm{C}\right)$. And used for further analysis. Optionally, a second denaturing elution was performed by addition of 1:1 SDS loading dye $5 \mathrm{~min}$ incubation at $\left(95^{\circ} \mathrm{C}\right)$ and subsequent centrifugation ( $1 \mathrm{~min} /$ 200 g/ RT).

\subsubsection{Activation and equilibration of IgG-Sepharose beads}

In order to activate IgG-Sepharose beads for protein isolation assays beads were first washed twice with a 10x excess of acetate buffer (0.5 M HAc, $0.5 \mathrm{M} \mathrm{NH}_{4} \mathrm{Ac}$, pH 3.4). Beads were then equilibrated to the used solubilization buffer washing twice with 10x excess of $2 x$ Solublization buffer and once with 10x excess of digitonin solubilization buffer.

Tab. 2.21 2x Solubilization buffer

\begin{tabular}{|ll|}
\hline Component & Concentration \\
Tris pH 7.4 & $40 \mathrm{mM}$ \\
$\mathrm{NaCl}$ & $2.4 .18 .180 \mathrm{mM}$ \\
Glycerol & $20 \%$ \\
EDTA & $2 \mathrm{mM}$ \\
\hline
\end{tabular}


Tab. 2.22 Digitonin solubilization buffer

\begin{tabular}{|l|l|}
\hline Component & Concentration \\
Tris pH 7.4 & $20 \mathrm{mM}$ \\
$\mathrm{NaCl}$ & $2.4 .18 .280 \mathrm{mM}$ \\
Glycerol & $10 \%$ \\
EDTA & $1 \mathrm{mM}$ \\
Digitonin & $1 \%$ \\
PMSF & $2 \mathrm{mM}$ \\
\hline
\end{tabular}

Tab. 2.23 Washing buffer

\begin{tabular}{|ll|}
\hline Component & Concentration \\
Tris pH 7.4 & $20 \mathrm{mM}$ \\
$\mathrm{NaCl}$ & Varying (see Results) \\
Glycerol & $10 \%$ \\
EDTA & $1 \mathrm{mM}$ \\
Digitonin & $0.3 \%$ \\
PMSF & $1 \mathrm{mM}$ \\
\hline
\end{tabular}

\subsubsection{Affinity purification with Glutathione Sepharose}

To isolate proteins tagged with Glutathione S-transferase (GST). Corresponding E. coli cell were harvested after $4 \mathrm{~h}$ expression by centrifugation (5 min/ $4500 \mathrm{~g}$ ) $\left.4{ }^{\circ} \mathrm{C}\right)$. Pellets were resuspended in PBS buffer (0,5 ml / $100 \mathrm{mg}$ cells) and subjected to sonication on ice. Volumes below $2 \mathrm{ml}$ were therefore exposed to 3 times 30 pulses in an ultrasound bath (Branson) within a small reaction tube while higher volumes were sonicated for 3 times 45 seconds with a sonication needle (Heat Systems). Solutions were clarified by cencentrifugation ( $30 \mathrm{~min} / 4500 \mathrm{~g} / 4{ }^{\circ} \mathrm{C}$ ) and upernatants were subsequently added to equilibrated Glutathione Sepharose beads $(50 \mu$ beads / $800 \mu \mathrm{l}$ ). For respective equilibration the Sepharose was sedimented by centrifugation at ( $5 \mathrm{~min} / 500 \mathrm{~g}$ ) and washed twice with 5 times excess of PBS buffer before. After an incubation overnight on an end-over-end shaker at $4{ }^{\circ} \mathrm{C}$ unbound proteins were removed by centrifugation (30 s/ $100 \mathrm{~g} /$ $4{ }^{\circ} \mathrm{C}$ ) and beads were washed 6 times with 5 fold excess of PBS buffer. For competitive elution, beads were incubated with $50 \mathrm{mM}$ Tris/HCl pH $8(100 \mu \mathrm{l} / 50$ $\mu \mathrm{l}$ beads) containing $10 \mathrm{mM}$ reduced Glutathione (GSH) for $5 \mathrm{~min}$ at room 
temperature with agitation, whereupon GSH-eluates were spin out (1 min/ $100 \mathrm{~g}$ / $4{ }^{\circ} \mathrm{C}$ ). A final denaturing elution (SDS eluates) was performed with SDS Loading Dye for $5 \mathrm{~min}$ at $95^{\circ} \mathrm{C}$ followed by centrifugation ( $1 \mathrm{~min} / 100 \mathrm{~g} / \mathrm{RT}$ ).

Tab. 2.24 PBS buffer pH 7.4

\begin{tabular}{|ll|}
\hline Component & Concentration \\
$\mathrm{NaCl}$ & $140 \mathrm{mM}$ \\
$\mathrm{KCl}$ & $27 \mathrm{mM}$ \\
$\mathrm{Na}_{2} \mathrm{HPO}_{4}$ & $101 \mathrm{mM}$ \\
$\mathrm{KH}_{2} \mathrm{PO}_{4}$ & $18 \mathrm{mM}$ \\
$\mathrm{PMSF}$ & $2 \mathrm{mM}$ \\
Protease Inhibitor EDTA free & 1 tablet / $50 \mathrm{ml}$ \\
\hline
\end{tabular}

\subsubsection{Mdm38K BpA in vivo cross-linking}

To investigate proteins in close proximity to different positions at the ribosomal binding domain of $\mathrm{Mdm} 38$ an in vivo cross-linking via $p$-benzoylphenylalanine (pBpA) was performed in E. coli according to the principle introduced by (Farrell et al. 2005). For the integration of pBpA expression vectors encoding constructs with an amber-stop (TAG) codon at intended positions were used. Furthermore the E. coli stains were co-transformed with the plasmid pSUP, which encodes the amber suppressor pBpA-tRNA and respective tRNA synthetase to enable the integration of $\mathrm{pBpA}$ at TAG-codons.

The whole assay was performed under minimization of light exposition of the samples to prevent background cross-links.

Precultures of $5 \mathrm{ml} \mathrm{LB}$ media supplied with $100 \mu \mathrm{g} / \mathrm{ml}$ ampicillin and $35 \mu \mathrm{g} / \mathrm{ml}$ chloramphenicol to select for both plasmids were inoculated with a single bacterial colony and grown with agitation at $30^{\circ} \mathrm{C}$. Next day main cultures of identical medium (negative control) or medium additionally containing $1 \mathrm{mM}$ pBpa were adjusted to $0.1 \mathrm{OD}_{600}$ and grown to 0.6 OD 600 . Expression was then induced with $0.5 \mathrm{mM}$ IPTG and cultures were further incubated for $4 \mathrm{~h}$ at $30^{\circ} \mathrm{C}$ under agitation. Cultures were put on ice and cells were subsequently harvested by centrifugation $\left(5 \mathrm{~min} / 5000 \mathrm{~g} / 4^{\circ} \mathrm{C}\right.$ ). Pellets were resuspended in PBS buffer (Tab. $2.24-2 \mathrm{ml}$ per $20 \mathrm{ml}$ main culture) and subsequently split into two equal volumes. Both samples were stored $30 \mathrm{~min}$ on ice. While one sample was exposed to UV light the other 
was kept in darkness as negative control. A custom-made UV chamber applied with a halogen metal vapor lamp (Osram Ultratech) with a glass screen filtering out protein damaging wavelengths below $300 \mathrm{~nm}$ was used here. In the following step cells were lysed by sonication as described in 2.4.19. After removal of insoluble components by centrifugation (30 min/ $5000 \mathrm{~g} / 4^{\circ} \mathrm{C}$ ) supernatants were used for further analysis.

\subsubsection{In vitro cross-linking to Mdm38K}

In order to test the availability of cysteine 315 of Mdm38K for externally added cross-linking reagents Alexa Fluor $\AA 488$ C5 Maleimide was added to purified Mdm38K as introduced by Yahaf et al. (2003).

The assay was performed in $50 \mu$ reaction buffer $(10 \mathrm{mM}$ EDTA, $40 \mathrm{mM}$ Tris/HCl $\mathrm{pH}$ 7.4) using $25 \mu \mathrm{M}$ of protein. The maleimide cross-linker was dissolved in Dimethylformamid (Sigma) an added in fivefold or tenfold excess including a mock control. Positive control were performed in parallel using reaction buffer with $2 \%$ SDS. Then, samples were kept at room temperature to allow cross-linking. Subsequently the reaction was quenched by the addition of $1 \mu \mathrm{l} 1 \mathrm{M}$ DTT. After additional $15 \mathrm{~min}$ at room temperature, loading dye was added to the samples. Following $5 \mathrm{~min}$ incubation at $95{ }^{\circ} \mathrm{C}$, samples corresponding to approximately 3 mg protein $(20 \mu \mathrm{l})$ were separated via SDS-PAGE. Corresponding gels were blotted or stained with coomassie to detect fluorescent signal via a flourescence scanner or show whole protein levels, respectively.

\subsubsection{Isolation of biotinylated proteins from mitochondria}

Following the principle introduced by (Roux et al. 2012) which was further adapted to yeast by Levchenko et al. (2015, unpublished) biotinylated proteins were isolated from yeast mitochondria. Therefore, cells with chromosomally integrated gene constructs encoding fusion proteins with a $\mathrm{N}$-terminally added biotin ligase, BirA were used for isolation of mitochondria (2.4.11).

After thawing on ice $1000 \mu \mathrm{g}$ mitochondria centrifuged $\left(10 \mathrm{~min} / 14.000 \mathrm{~g} / 4^{\circ} \mathrm{C}\right)$. Resulting pellets were subsequently resuspended in $1000 \mu \mathrm{l}$ RIPA lysis buffer. Following $5 \mathrm{~min}$ incubation on ice lysates were clarified by centrifugation (10 min/ $14.000 \mathrm{~g} / 4^{\circ} \mathrm{C}$ ). After samples were taken (Total) supernatant was transferred to $40 \mu \mathrm{l}$ Streptavidin agarose beads that were previously equilibrated twice with 
RIPA lysis buffer. Following $2 \mathrm{~h}$ rotation on an end-over-end shaker at $4{ }^{\circ} \mathrm{C}$ beads were cleared from unbound (Unbound sample) material by centrifugation (30 s/ $100 \mathrm{~g} / 4^{\circ} \mathrm{C}$ ). 5 washing steps were performed using $300 \mu$ l RIPA lysis buffer for the first and the last two steps and $2 \%$ SDS for the third one. Finally columns were dried and denaturing elution was performed by addition of $100 \mu$ l hot SDS loading dye and subsequent 5 min incubation at $95^{\circ} \mathrm{C}$. Samples were spin out by centrifugation (1 $\mathrm{min} / 200 \mathrm{~g} / \mathrm{RT}$ ).

Tab. 2.25 RIPA lysis buffer

\begin{tabular}{|ll|}
\hline Component & Concentration \\
TRIS (pH 8.0) & $50 \mathrm{mM}$ \\
$\mathrm{NaCl}$ & $150 \mathrm{mM}$ \\
$\mathrm{SDS}$ & $0,1 \%$ \\
Sodium Deoxycholat & $0,5 \%$ \\
TritonX100 & $1 \%$ \\
PMSF & $1 \mathrm{mM}$ \\
Na Azide & $10 \mathrm{mM}$ \\
EDTA free Protease inhibitor & 1 tablet/ $50 \mathrm{ml}$ \\
\hline
\end{tabular}

\subsubsection{Mass-spectrometry}

Samples for analysis by mass sprectrometry were excised from SDS gels stained with colloidal coomassie and transferred to an Eppendorf cup. The gel piece was subsequently covered with $100 \mu \mathrm{l} 25 \mathrm{mM} \mathrm{NH}_{4} \mathrm{HCO}_{3}$ and incubated (a) for $15 \mathrm{~min}$ at $37^{\circ} \mathrm{C}$ with fast agitation (1000 rpm), followed by further incubation steps of $30 \mathrm{~min}$ (same coditions ) in (b) $25 \mathrm{mM} \mathrm{NH}_{4} \mathrm{HCO}_{3} 25$, (c) $25 \mathrm{mM} \mathrm{NH}_{4} \mathrm{HCO}_{3} / 50 \%$ acetonitrile, (d) 100\% acetonitrile (e) $10 \mathrm{mM}$ dithiothreitol and in $25 \mathrm{mM}$ $\mathrm{NH}_{4} \mathrm{HCO}_{3}$ at $56^{\circ} \mathrm{C}$ for $1 \mathrm{~h}$ for final reduction. After repetition of al previous incubation steps (a-e) final carbamidomethylation was performed in $25 \mathrm{mM}$ indoacetamide, $25 \mathrm{mM} \mathrm{NH} 4 \mathrm{HCO}_{3} / \mathrm{H}_{2} \mathrm{O}$ at $56^{\circ} \mathrm{C}$. After overnight digestion with trypsin at $37^{\circ} \mathrm{C}$ proteins were extracted. Therefore, samples were incubated for 30 $\min$ at $37{ }^{\circ} \mathrm{C}$ in $1 \%$ trifluoroacetic acid (TFA) and dried in a vacuum concentrator (SpeedVac). Gel pieces were resolved in $10 \mu \mathrm{L}$ 0.1\% TFA were added and subsequently vortexed and sonified. Achieved solutions were finally applied to nano-LC-MALDI-MS/MS analysis. 
Results were assumed as true for identified proteins if at least two different fragments with an expectation value below 0.5 were detected.

Sample preparation and data analysis were performed by Olaf Bernhard (Göttingen). 


\section{Results}

\subsection{Ribosomal Binding Domain of Mdm38}

\subsubsection{Binding to mitochondrial ribosomes in yeast is salt dependent}

Mdm38 is a protein of about $65 \mathrm{kDa}$, localized at the matrix side of the inner mitochondrial membrane. Earlier studies suggested a role for Mdm38 in the export of newly synthesized, mitochondria-encoded proteins into the inner membrane (Frazier et al. 2006) and also for mitochondrial translation (Bauerschmitt et al. 2010). In line with a functional connection to translation, interactions between mitochondrial ribosomes and Mdm38 were revealed by co-isolation experiments and resulted in the identification of a ribosomal binding domain (Lupo et al. 2011). In order to confirm previous findings and to characterize the interaction between Mdm38 and mitochondrial ribosomes more specifically, affinity purification using IgG-Sepharose was performed (2.4.17). Mitochondria were isolated (2.4.11) from yeast expressing Mdm38 with a chromosomal C-terminal Protein A (ProtA) fusion (Mdm38 ${ }^{\text {ProtA }) ~(F r a z i e r ~ e t ~ a l . ~ 2006) . ~ M i t o c h o n d r i a ~ w e r e ~ s o l u b i l i z e d ~ w i t h ~} 1 \%$ digitonin and subsequently incubated with IgG-Sepharose beads. To examine the binding characteristics of Mdm38, samples were split and washed with buffers containing different salt concentrations ( $80 \mathrm{mM} \& 350 \mathrm{mM} \mathrm{NaCl}$ ). Finally, samples were eluted by cleavage of the ProtA-tag with TEV-protease, followed by a second elution using SDS-loading dye. TEV-protease elutions were then dissolved in SDS loading dye. Western Blot analysis (Figure 3.1) revealed an enrichment of mitochondrial ribosomal proteins (Mrpl4 \& Mrpl39) in both elutions after low salt washing, whereas co-purified amounts were significantly lower after washing using $350 \mathrm{mM} \mathrm{NaCl}$. Tom 20 was used as a negative control, since this protein of the outer mitochondrial membrane is not associated with Mdm38 and therefore shows no unspecific binding. Respectively, Ylh47 was used as a positive control, whereby co-isolation with Mdm38 was shown by Frazier et al.. Overall, these results are in line with (Frazier et al. 2006) and suggest an ionic interaction of Mdm38 and yeast mitochondrial ribosomes. 


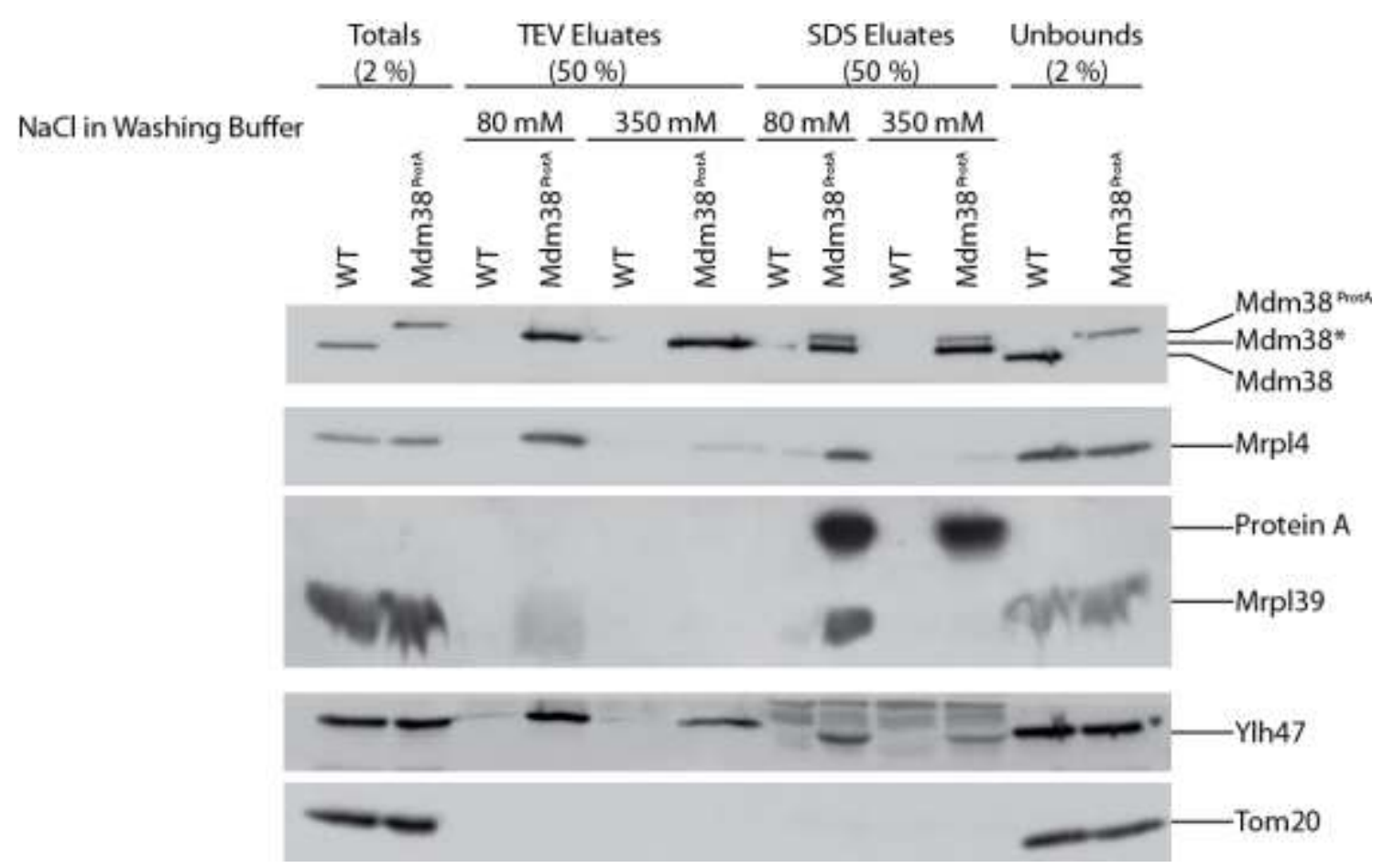

Figure 3.1 Salt-dependent co-isolation of mitochondrial proteins with Mdm38 ${ }^{\text {ProtA }}$ Lysates of $1 \mathrm{mg}$ mitochondria from wild-type or Mdm38 ProtA yeast strains were incubated with IgG-Sepharose. Samples were subsequently split and beads were washed using buffer with different $\mathrm{NaCl}$ concentrations ( $80 \mathrm{mM} \& 350 \mathrm{mM}$ ). Bound proteins were eluted with TEV-protease and subsequently with SDS loading dye. The corresponding SDS-gel was blotted and immunostained for indicated proteins. Mdm38* marks Mdm38 ${ }^{\text {ProtA }}$ after TEV-cleavage and Protein A the corresponding cleaved tag. Next to mitochondrial ribosomal proteins (Mrpl4 \& Mrpl39), Ylh47 was used as a positive and Tom20 as a negative control.

\subsubsection{Binding to $E$. coli ribosomes shows conserved characteristics}

The structure of the C-terminal domain of Mdm38 (CTD - aa 159-573) was recently determined (Lupo et al. 2011). Therefore, a construct, which was C-terminally tagged with His6 and N-terminally tagged with Glutathione S-transferase (Mdm38CTD - Figure 3.2 A), was expressed in E. coli. Samples were then purified and subjected to x-ray crystallography. The results of Lupo et al. indicated that stable crystals only consist of a smaller fragment (aa 182-408), termed Mdm38 RBD or Mdm38K. Additional experiments using truncations in yeast and recombinant constructs expressed in E. coli (Mdm38K- Figure 3.2 A) suggested, that this part of the protein is the ribosomal binding domain.

Based on these findings, the constructs mentioned above were expressed in E. coli using IPTG inducible vectors; pGex4T2 Mdm38K and pGex4T3 Mdm38CTD (2.1.6) and an empty vector (pGex4T2), expressing GST, was used as a control. 
Subsequently, cells were subjected to affinity purification with glutathione (GSH) Sepharose (2.4.19) and the corresponding samples were separated by SDS-PAGE (Figure 3.2 B). A coomassie stained gel enabled visualization of enriched expression products as prominent stained bands.

In addition, the Western Blot of a replica gel was immunostained for the ribosomal E. coli protein L23, which was significantly enriched in both constructs, but not the negative control. In conclusion, these results confirm previous findings and demonstrate that binding of $\mathrm{Mdm} 38 \mathrm{~K}$ is also true for E. coli ribosomes.

A)

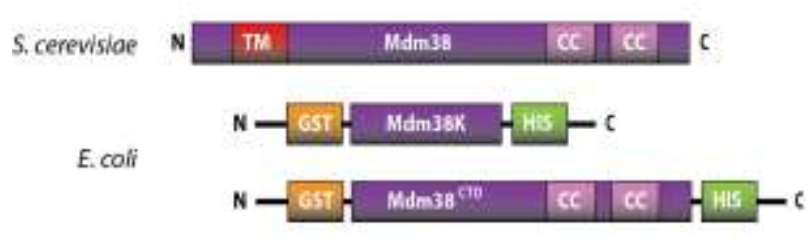

B)

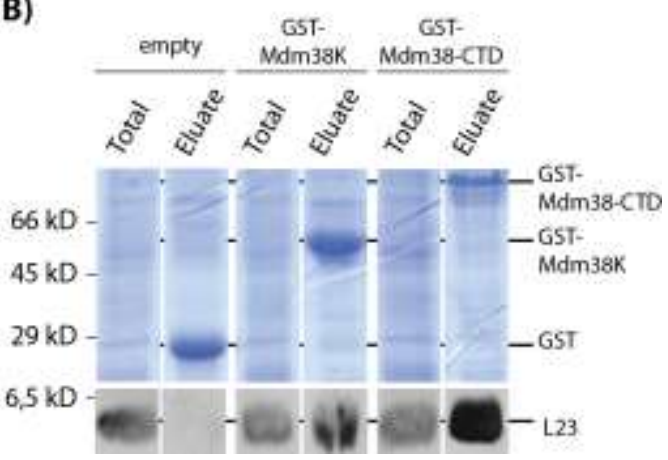

Figure 3.2 Co-isolation of $E$. coli ribosomes using recombinant expressed constructs of Mdm38

A) Shown are cartoons of fused tagged constructs expressed in E. coli. The yeast protein is depicted for comparison, whereas predicted transmembrane- $(\mathrm{T})$ and coiled-coil (CC) domains are indicated.

B) The upper part of the figure shows a coomassie stained SDS gel of an affinity purification with Glutathione Sepharose, whereas samples were eluted with SDS loading buffer. Indicated constructs were purified from cells after $4 \mathrm{~h}$ expression. The empty vector expressing the GST-tag only was used as a control. The lower part shows the Western Blot of the same experiment immune decorated for the ribosomal E. coli protein L23.

In order to study the binding characteristics of Mdm38 to bacterial ribosomes, the salt sensitivity was investigated (Figure 3.3). Therefore, isolations of GST-constructs were performed as described above. Deviating from the previous procedure, GSH beads were washed with a high salt buffer, containing $350 \mathrm{mM} \mathrm{NaCl}$. Subsequently, samples were split and sequential elutions were performed with GSH and SDS loading dye for $50 \%$ of the Sepharose.

The lack of signals for specifically enriched L23 in the eluates suggest a salt dependent binding, which was previously described for yeast ribosomes (Figure 3.4 I). 


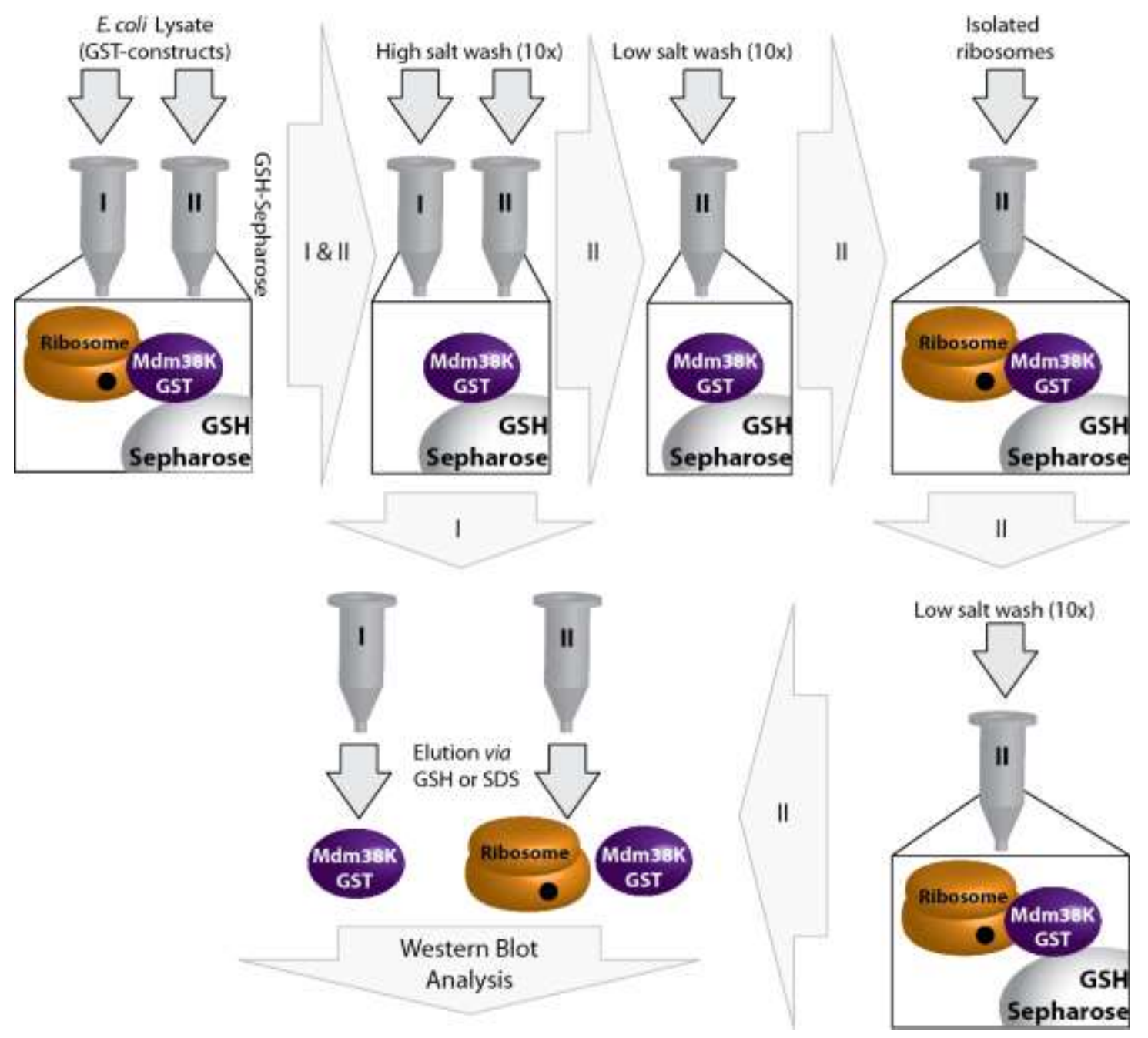

Figure 3.3 Removal and rebinding of ribosomes to immobilized Mdm38K

Shown is a schematic representation of the steps performed for in vitro binding of ribosomes to immobilized GST-constructs exemplified for Mmd38-GST. High salt buffer contains $350 \mathrm{mM} \mathrm{NaCl}$ and low salt buffer contains $140 \mathrm{mM} \mathrm{NaCl}$.

To confirm the hypothesis of salt-sensitive binding, in vitro binding was tested for the other half of washed beads (Figure 3.4 II). Sepharose was re-equilibrated to a lower $\mathrm{NaCl}$ concentration $(140 \mathrm{mM})$ and isolated E. coli ribosomes (kindly provided by the group of Prof. Dr. Marina Rodnina) were added. These ribosomes were hereby adjusted to ribosome concentrations within the cell lysate, estimated in preliminary tests using Western Blots ( $\sim 50 \mathrm{pM}$, data not shown). The corresponding Western Blots displays specifically enriched signals for L23 in the elutions of both constructs, in comparison to the control. Furthermore, signals for unbound mitochondria indicate similar ribosomal concentrations as for lysed $E$. coli and the added isolates. 
In conclusion, these results suggest a salt sensitive binding of Mdm38K and Mdm38CTD to ribosomes of $E$. coli, which was hitherto solely described for S. cerevisiae. To this end, a conserved binding mechanism is assumed.

A)

B)

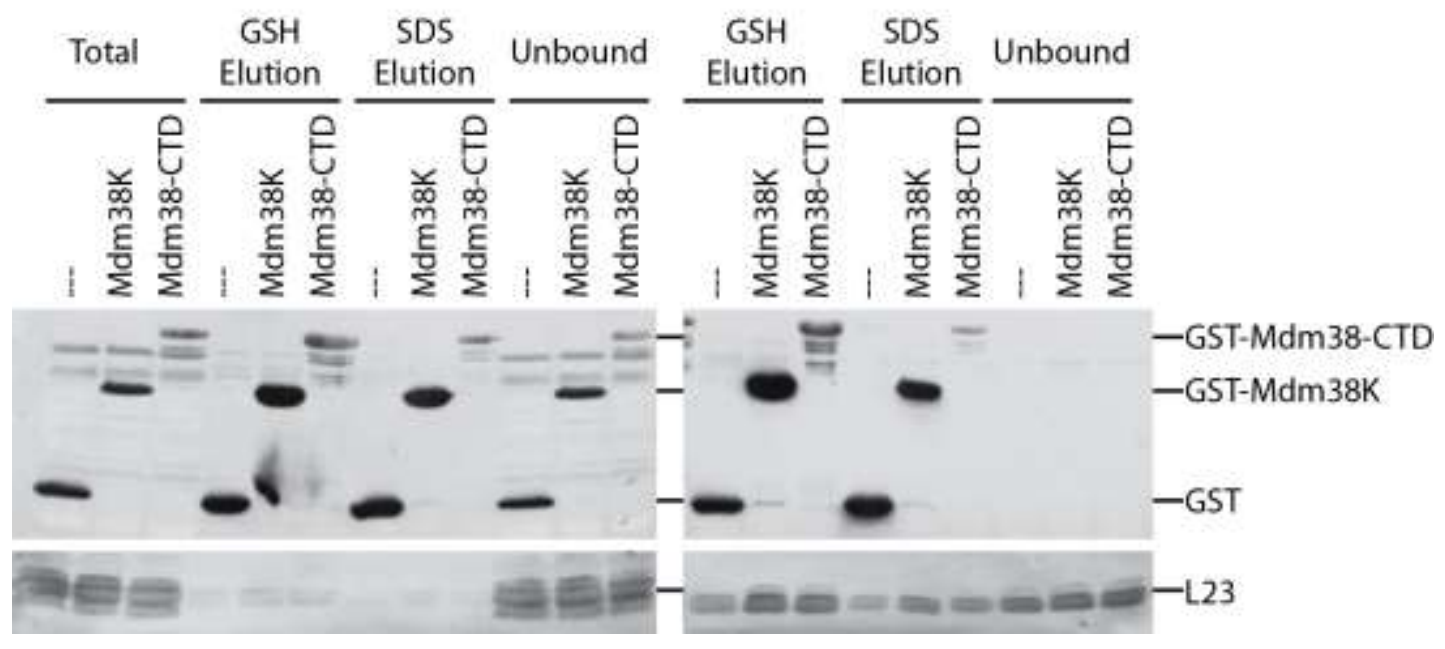

Figure 3.4 Salt-dependent binding to $E$. coli ribosomes

Western Blots immune stained for GST (top part) and L23 (bottom part)

A) Isolation of GST-tagged constructs was performed as for Figure 3.2 whereas Sepharose beads were washed with high salt buffer $(350 \mathrm{mM}$ $\mathrm{NaCl}$ ) exhibiting reduced amounts of ribosomal protein in corresponding eluates.

B) Sepharose was equilibrated to a lower salt concentration $(140 \mathrm{mM} \mathrm{NaCl})$ after high salt wash (I). Subsequently, beads were incubated with isolated $E$. coli ribosomes for $1 \mathrm{~h}$, whereupon unbound ribosomes were removed. Corresponding elutions exhibit L23 protein, specifically enriched for both constructs.

\subsection{Establishing in vivo photo cross-linking with $\mathrm{pBpA}$}

Given the above results, binding of the recombinant Mdm38K domain to yeast and E. coli ribosomes might follow similar mechanisms. However, interacting proteins and their respective binding properties remain to be characterized. To approach this issue, in vivo photo cross-linking was established (Chin et al. 2002). This assay enables the identification of proteins in close proximity $(\sim 6.5 \AA$ from the C $\alpha$ carbon (Banta et al. 2014) ) to specific positions within a target protein. Therefore, amino acids were exchanged with the photo cross-linkable artificial amino acid para-benzoylphenylalanine (pBpA), by expansion of the genetic code.

Since the crystal structure of Mdm38K revealed a highly conserved cavity that was assumed to be involved in protein-protein interactions (Lupo et al. 2011), 
positions within that area were chosen for pBpA incorporation (amino acids: C315, M320, S354, L330, G319 \& W337).
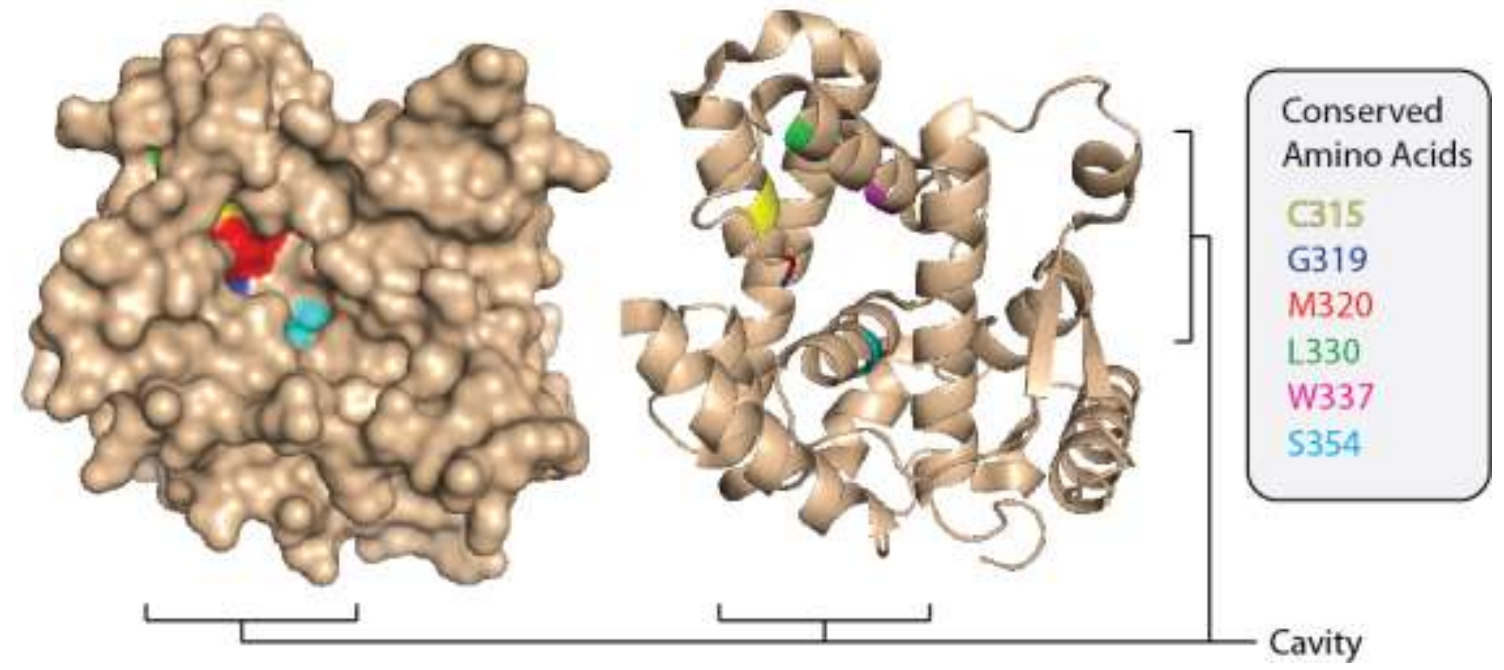

Figure 3.5 Positions for pBpA incorporation

A surface (left) and a ribbon model (right) depicting the 3D-structure of the ribosomal binding domain of Mdm38. Conserved amino acids chosen for pBpA exchanges are colored and the position of the assumed binding cavity is indicated.

Illustrations were created with Pymol (Schrödinger, LLC) using structural data published by Lupo et al., 2011.

\subsubsection{Mdm38 derivatives with alanine exchanges are functional}

All amino acids chosen for pBpA exchange were highly conserved. Thus, they are most likely to be essential for the function of the domain. To exclude any severe effects resulting from these exchanges, further analyses were performed in $S$. cerevisiae. Therefore, complementation of $m d m 38 \Delta$ mutant phenotypes was tested with chromosomally expressed Mdm38 mutants, in which the respective amino acids are exchanged to alanine.

For this purpose, Mdm38 was cloned into the yeast plasmid pRS416, together with its endogenous promoter. After verification, achieved constructs were used for mutagenesis, generating amino acid exchanges to alanine at specific positions (2.3.8 - Primers: Tab. 2.5). Verified constructs were transformed into wild-type and $m d m 38 \Delta$ yeast strains. Empty plasmids and plasmids expressing wild-type Mdm38 were transformed as controls.

To determine the functionality of these Mdm38 derivatives, growth tests were performed (2.2.3). Serial dilutions were spotted on solid selective media containing either glucose or glycerol as the carbon source (Figure $3.6 \mathrm{~A}$ ). The $m d m 38 \Delta$ strain containing the empty plasmid shows the characteristic reduced 
growth on non-fermentable media. In contrast, all strains transformed with plasmids expressing either wild-type or mutagenized Mdm38 display growth comparable to the wild-type control with empty plasmid.

A)

Background pRS416

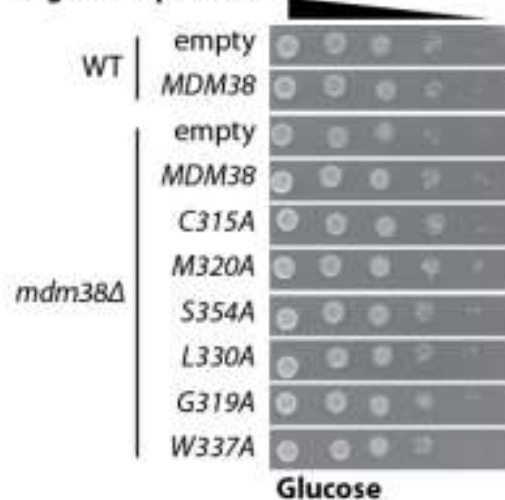

B)

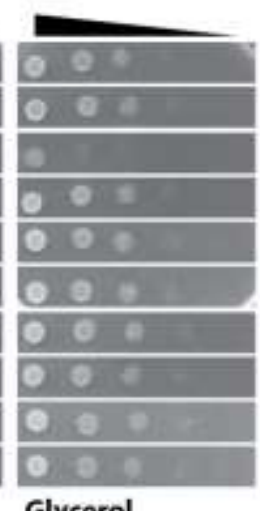

Glycerol

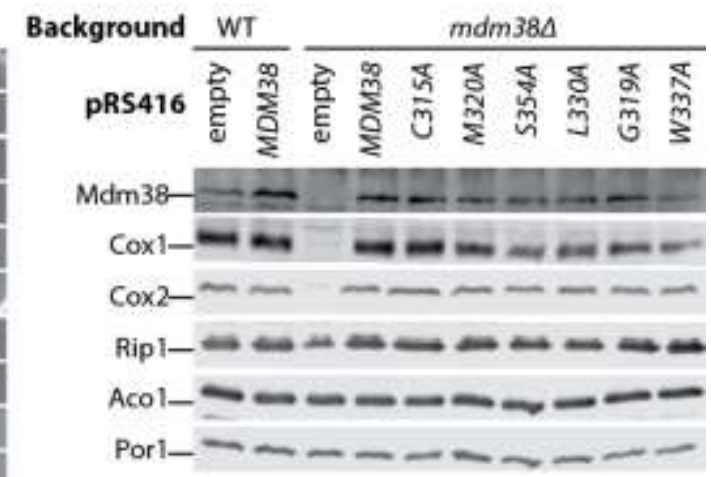

Figure 3.6 Analysis of alanine exchanges

A) Shown is a growth test on fermentable and non-fermentable synthetic media lacking uracil. Cells were spotted in serial 10 -fold dilutions and incubated at $24^{\circ} \mathrm{C}$. Strains were transformed with pRS416 either empty or with different derivatives of MDM38 (see text).

B) Whole cell extracts of indicated yeast strains were separated by SDS-PAGE, followed by Western Blot and probed for various mitochondrial proteins using Aco1 and Por1 as loading controls.

Another phenotype of Mdm38 deficient strains is the reduced steady state levels of different mitochondrial proteins (Frazier et al. 2006). Therefore, whole cell extracts (2.4.9) of all strains used above were prepared. After separation using SDS-PAGE and subsequent Western blotting, protein levels were analyzed by immunostaining (Figure3.6 B). Reduced amounts of the respiratory chain proteins Cox1, Cox2 \& Rip1, which is characteristic for Mdm38 deficiencies (Frazier et al. 2006; Lupo et al. 2011), could only be detected for the deletion mutant (empty plasmid), whereas for all other strains wild-type levels were detected.

Thus, it was concluded that Mdm38 containing the conserved aa-exchanges within the ribosomal binding domain, as described above, is still functional.

\subsubsection{Creation of an $M d m 38 K$ construct for $p B p A$ incorporation}

Constructs of Mdm38K for in vivo cross-linking were designed to enable double purification with an N-terminal His14- and a C-terminal Strep-tag. Additionally, a SUMO (small ubiquitin related modifier) tag was chosen as a solubility tag and for potential cleavage of the HIS-tag ((Kuo et al. 2014) (illustrated in Figure 3.8 left branch). A suitable vector, pSUMO, for N-terminal tagging with His14-SUMO was 
kindly supplied by the group of Prof. D. Görlich (Göttingen). For cloning of Mdm38K, the intended fragment (aa 192-408) was amplified by PCR. Cloning was performed using introduced restriction sites and constructs were confirmed by restriction analysis. In the next step, codons for amino acids C315, M320, S354, L330, G319 \& W337 of Mdm38 were exchanged to amber-stop codons using site directed mutagenesis. Mutagenesis PCR was performed using primer pairs listed in (Tab. 2.5) and clones were verified by sequencing.

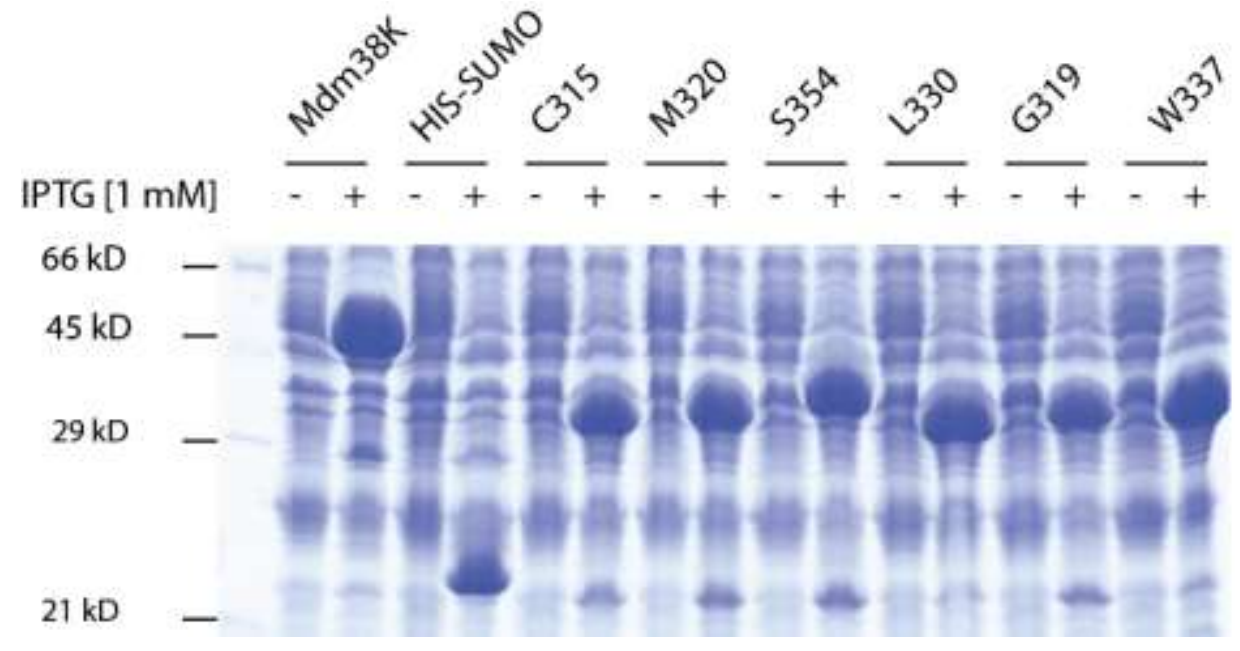

Figure 3.7 Expression of pSUMO constructs

Coomassie stained SDS-Gel of E. coli lysates. Strains are transformed with IPTG inducible expression vector pSUMO. The vector either contains no insert (HIS-SUMO) or derivatives of Strep-tagged Mdm38K, which were not mutagenized or contain an amber-stop replacement at the indicated position (amino acids: C315, M320, S354, L330, G319 \& W337). Samples were taken after $4 \mathrm{~h}$ incubation either without (-) or with expression (+) induced by 1 mM IPTG.

Furthermore, constructs were transformed (2.3.12) into the E. coli expression strain BL21 (2.1.8). Expression was induced by the addition of IPTG and resulting clones were tested for expression using a non-mutagenized construct as control. Non-induced samples of each strain (without IPTG) were used as controls. Samples were finally separated by SDS-PAGE. Coomassie staining of the gels revealed bands of highly expressed proteins of different sizes (Figure 3.7). Expression of unmodified Mdm38K results in a protein band with the expected size of $41.6 \mathrm{kDa}$. Furthermore, products of the amber-stop mutants, in which expression was terminated at their respective positions, correspond to sizes of around $31.4 \mathrm{kDa}$ for L330. The expression product of the empty vector fits to the size of the HISSUMO module $(18 \mathrm{kDa})$. It was therefore concluded that all constructs were correct and suitable for expression. 
The method of in vivo cross-linking in E. coli was already successfully performed in the group of Prof. Georg Koch (Freiburg). There, efficient pBpA insertion during expression of peptides is only efficient for lower expression rates. For this purpose, the use of vectors pTRc99a or pBad24 is well established and so the constructs described above were cloned into these expression plasmids. Since no restriction sites were available at the multiple cloning site (MCS) of pTRc99a or pBad24, and were also not contained within the sequence of Mdm38K constructs, a multi-step cloning strategy was used (schematically outlined for pTRc99a in Figure 3.8). First, the HIS-SUMO module was cloned from pSUMO into both other plasmids. Then, all Mdm38K constructs described above were transcloned from the respective pSUMO variants into both newly generated plasmids.

For further investigation of pTRc99a and pBad24, plasmids were transformed into the E. coli expression strains BL21 and C43. Additionally, pSUP was cotransformed in the same procedure to enable later incorporation of pBpA. Therefore, all following assays were performed in double selective media (LB + $100 \mathrm{mg} / \mathrm{ml} \mathrm{Amp}+35 \mathrm{mg} / \mathrm{ml}$ chloramphenicol). Finally, expression was induced, followed by Western Blot analysis of lysed cells. His-Probe-HRP (Thermo Fisher Scientific) was used for detection of His-tagged constructs (2.4.5). 


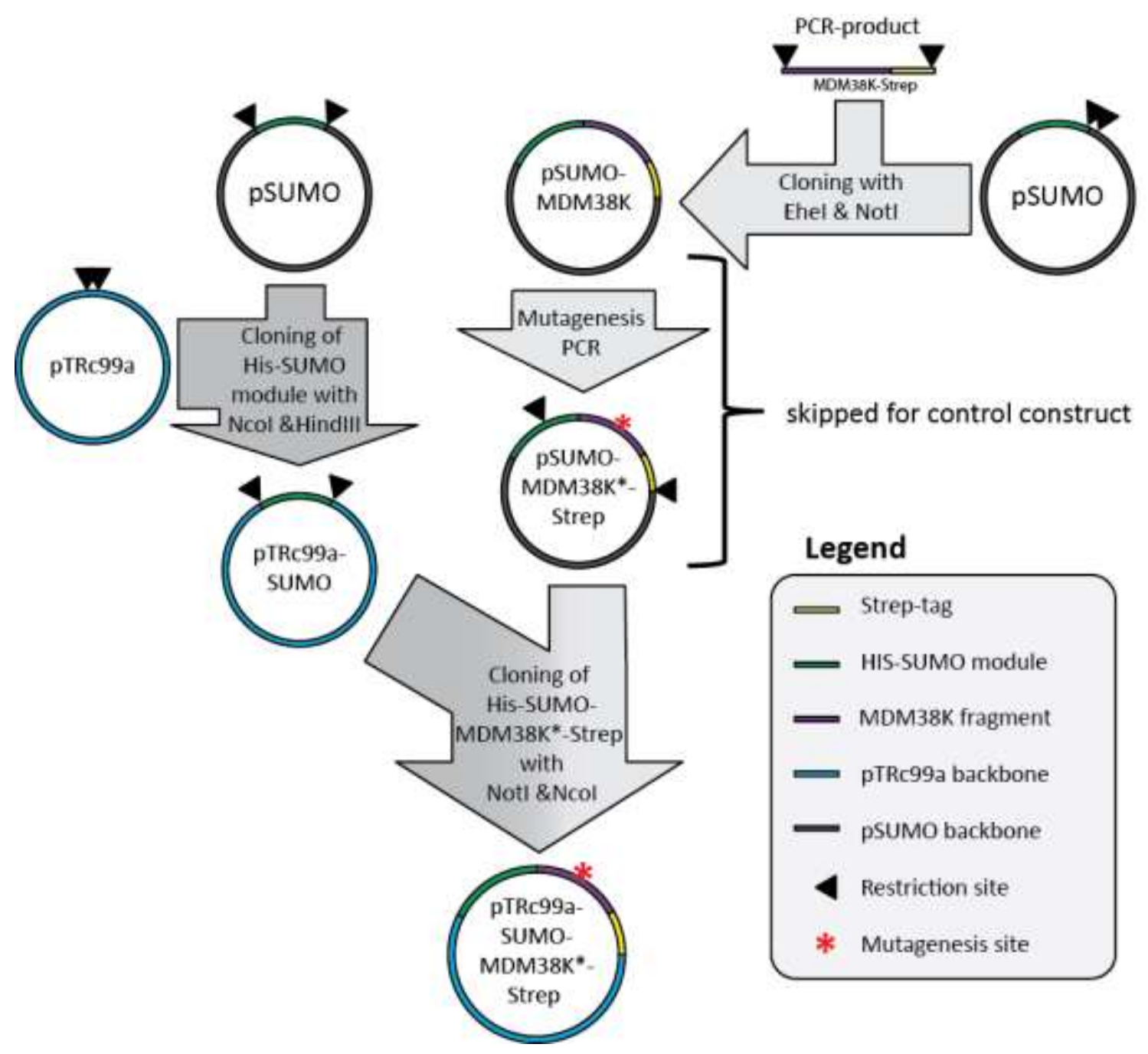

Figure 3.8 Cloning strategy for Mdm38K constructs

Schematic illustration of the construction strategy for the expression of His14-SUMOMdm38K-Strep construct using pTRc99a.

Induction of strains transformed with variants of pTRc99a resulted in peptides of expected size for all constructs but the empty vector (Figure 3.9). It is worth noting that achieved amounts were much lower than the expression products of pSUMO and thus not distinguishable from background proteins on coomassie stained gels (not shown), but only on Western Blots. Altogether, constructs in pTRc99a were considered to be suitable for further assays. 

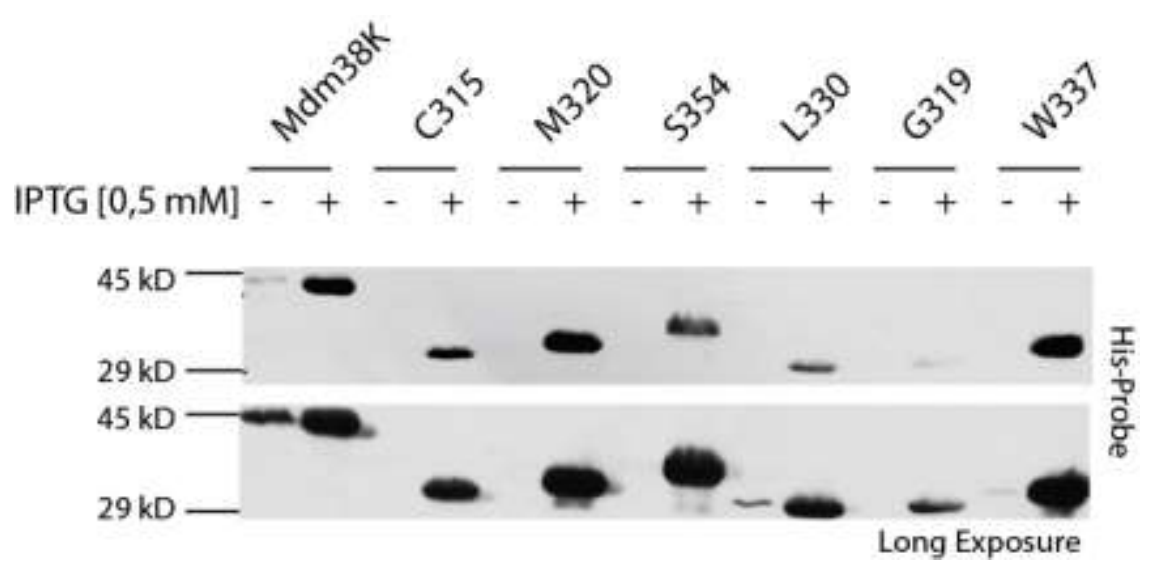

Figure 3.9 Expression products of pTRc99a

Western Blot of cell lysates from BL21 cells containing different pTRc99a constructs stained with His-Probe-HRP. Positions of amber-stop exchanges were indicated whereas, the non-mutagenized construct, Mdm38K, was used as control.

Lysates correspond to $100 \mu \mathrm{l}$ culture with (+) or without (-) induction by $0,5 \mathrm{mM}$ IPTG for $4 \mathrm{~h}$ at $30^{\circ} \mathrm{C}$. To visualize expression products of different abundance a shorter and longer exposure of the same blot are depicted.

\subsubsection{Incorporation of pBpA into Mdm38K}

The integration of $\mathrm{pBpA}$ is performed by a technique extending the genetic code (Chin et al. 2002). An amber-suppressor t-RNA, charged with pBpA by its corresponding t-RNA synthetase (both encoded on pSUP), enables the integration of the artificial amino acid at the position of a UAG-codon. However, these codons not only exist at the intended position created by mutagenesis PCR, but also as natural stop-codons in $E$. coli. In consequence, expression in the presence of $\mathrm{pBpA}$ leads to the continued expression of several genes, in addition to the target gene, beyond their stop-codon within E. coli and thus to potentially harmful effects. However, the impact of different toxic proteins is reduced in the C43 strain and so this strain was chosen for expression (Miroux and Walker 1996).

As an initial approach, the incorporation of pBpA was confirmed by the synthesis of mutagenized constructs to full length. To induce expression, cells were grown in media containing $1 \mathrm{mM}$ pBpA. It is worth noting that M9-minimal medium, suggested by Chin et al., led to no detectable expression products of expected sizes (data not shown). Additionally, expressed products were enriched by affinity purification using Ni-NTA. As depicted in the Western Blot of the corresponding SDS-eluates, the wild-type Mdm38K construct was expressed to full length independent of the presence of pBpA. Whereas, cells with TAG-codons at positions M320, G319 and W337, exhibit expression products of prematurely terminated 
translation in the absence of $\mathrm{pBpA}$, as described before. Moreover, the absence of detectable amounts of full-length protein indicates that potential translation through the AUG-codon is, if occurring, only a very rare event. However, additional Western Blot bands, corresponding in size to the full-length construct were detectable when pBpA was added to the medium. In consequence, it was concluded that $\mathrm{pBpA}$ is incorporated and thus enables full length translation. It is worth mentioning, that a certain level of expression occured without induction by IPTG for all full length constructs shown here.

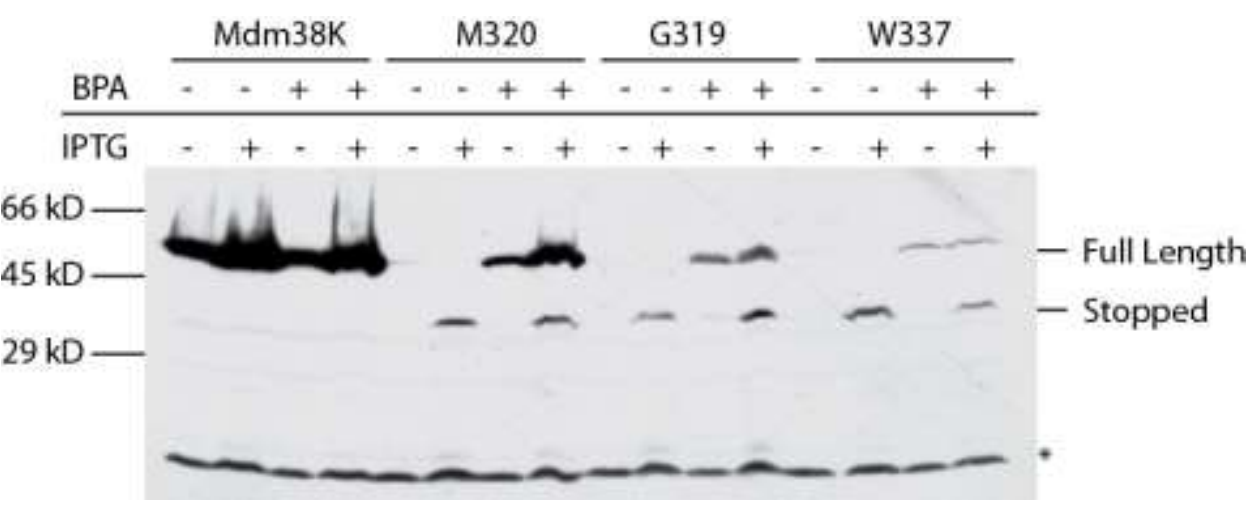

Figure 3.10 Incorporation of pBpA

Western Blot of SDS-eluates from His-purification with Ni-NTA probed with His-ProbeHRP. Samples were obtained from induced or uninduced cultures (+/- IPTG) with or without $\mathrm{pBpA}$. Four different constructs expressed from pTRc99 were loaded: nonmutagenized (Mdm38K) and 3 amber-stop exchanges at indicated positions (M320, G319 or W337).

The asterisk marks bands corresponding to a histidine rich $E$. coli protein Cat (Chloramphenicol acetyltransferase), which is therefore co-isolated and also stained at the Western Blot.

\subsubsection{In vivo photo cross-linking}

Since it has been shown that pBpA-incorporation was successful, strains expressing detectable amounts of pBpA-containing constructs ( $\mathrm{pBpA}$ at M320, G319 or W337) were finally used for the complete in vivo photo cross-linking approach (Schematically illustrated in Figure 3.11). Cells from induced cultures grown in pBpA containing media were exposed to UV light. This resulted in covalent binding of pBpA to C-atoms within $\sim 3 \AA$ A. Subsequently, cells were lysed and Mdm38K constructs were isolated using Ni-NTA or Strep Tactin beads. Different negative controls were applied in this context: The unmodified construct; cultures without $\mathrm{pBpA}$ and cells that were not exposed to UV-light. As depicted in Figure $3.12 \mathrm{~A}$, the construct containing pBpA instead of M320 leads to specific 
signals after UV exposure as visualized by Western Blot: one higher molecular weight band, detectable at $\sim 180 \mathrm{kDa}$ - (asterisk), and some potential smaller ones. Importantly, isolation was performed by the C-terminal Strep-tag using StrepTactin agarose beads. Therefore, only full length constructs, where translation was not stopped before the tag, were isolated.

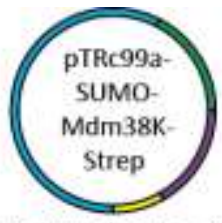

A) Control construct without exchange

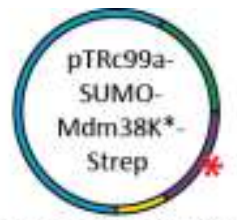

B) Constructs with amber stop

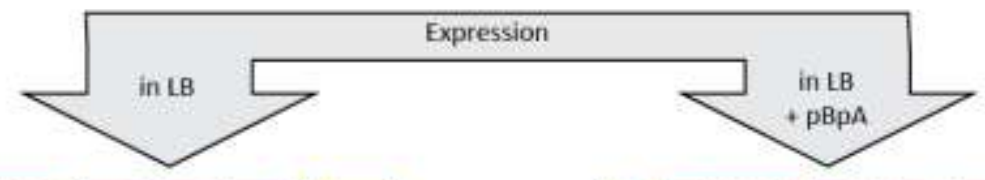

A)
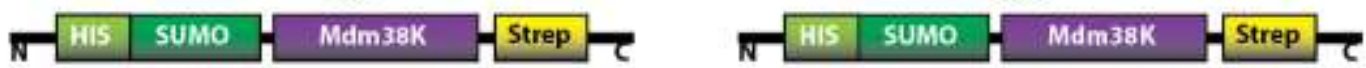

B)
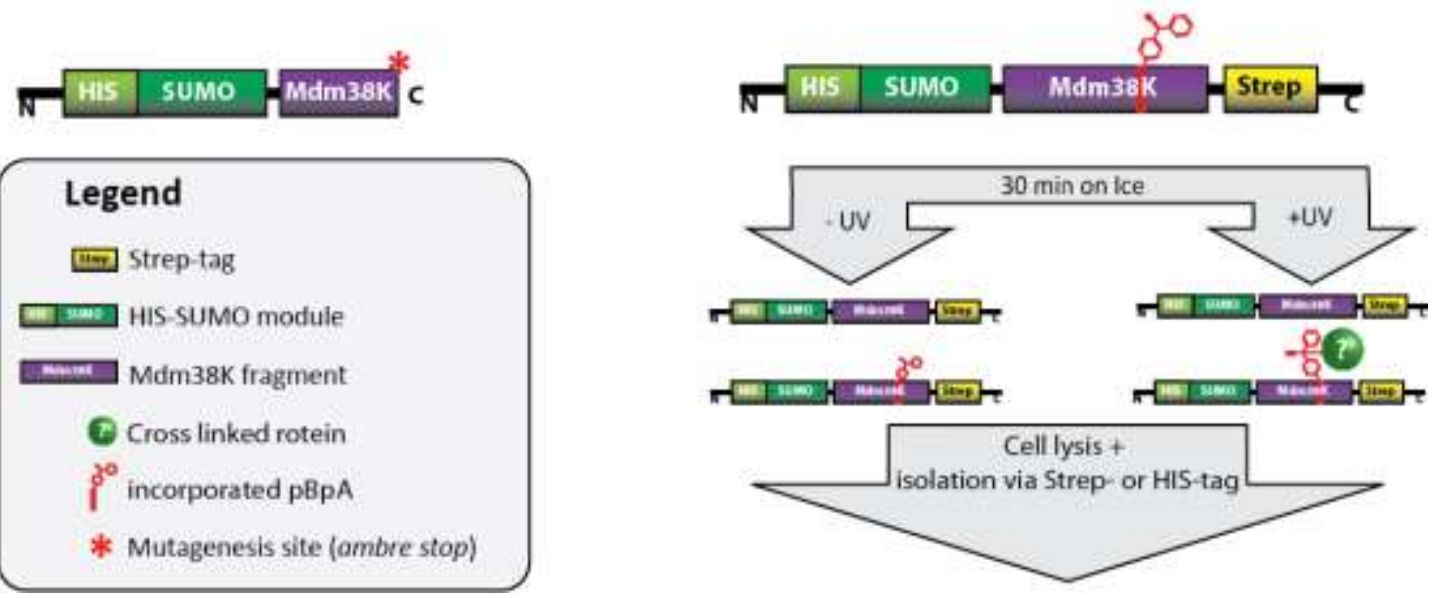

Figure 3.11 pBpA incorporation and photo cross-linking

Schematic illustration of performed steps to obtain Mdm38K-constructs cross-linked to potential interaction partners. 

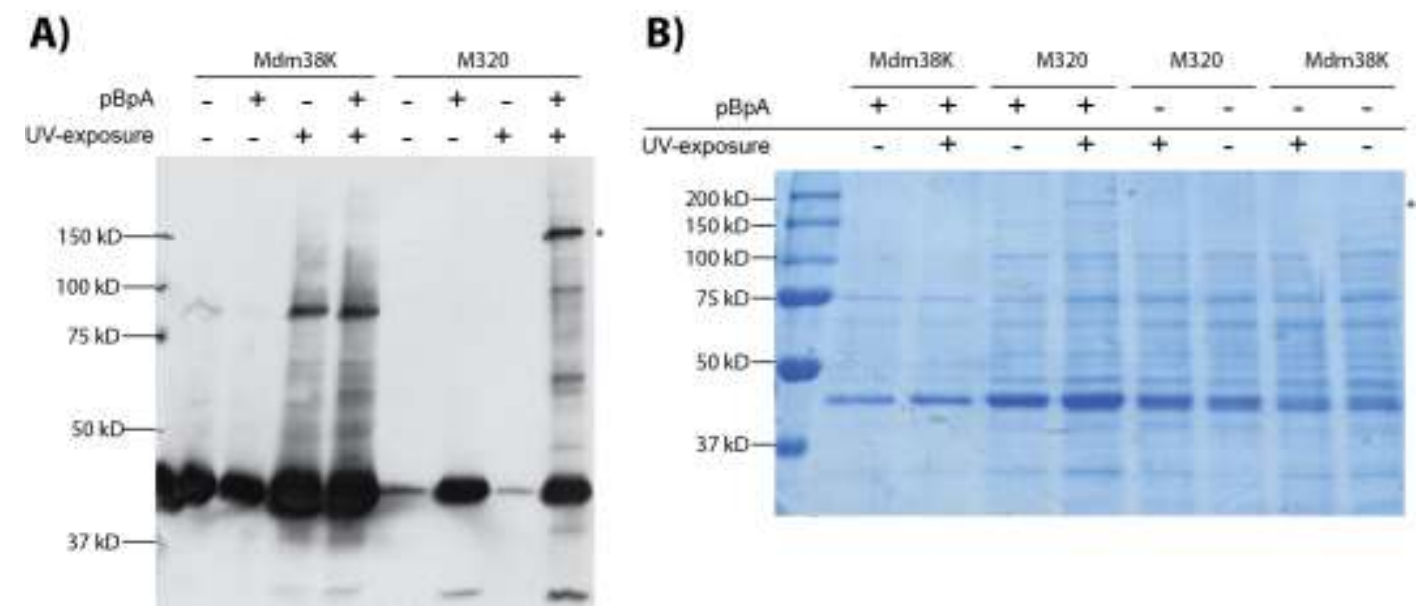

Figure 3.12 Cross-linking products

A) Expressions of Mdm38K-constructs were induced for $4 \mathrm{~h}$ in medium with and without $\mathrm{pBpA}$ using the E. coli C43 strain. Cells were subsequently exposed to UV-light, lysed and proteins were isolate with Strep-Tactin agarose beads. The corresponding Western Blot after SDS-PAGE was probed with His-Probe-HRP. The $+U V /+p B p A$ sample of the M320 exhibits specific bands. The most prominent signal is indicated by an asterisk right from the blot.

B) Cells were treated as in A, whereas protein isolation was performed with Ni-NTA beads. The corresponding SDS-gel was stained with colloidal coomassie. The asterisk indicates band size of a band specific for the $+\mathrm{UV} /+\mathrm{pBpA}$ sample of the M320, which was cut out and subjected to mass spectrometry.

Identification of the specific photo cross-linking band was finally performed via mass spectrometry (2.4.23). Cultures and corresponding controls were treated as described above. However, initial results (not shown) revealed higher levels of unspecific isolation products for Strep-Tactin agarose. For this reason, affinity purification was performed with Ni-NTA. Eluted samples were separated on an SDS-gel and subsequently stained with colloidal coomassie (2.4.7). As depicted in Figure $3.12 \mathrm{~B}$, compared to the previous result (Fig. $3.12 \mathrm{~A}$ ), a higher molecular weight band became visible, which was subsequently cut and analyzed by mass spectrometry. Analysis detected the yeast proteins Mdm38 and Smt3 (corresponding to the SUMO-tag) in the sample as well as several E. coli proteins (listed in Tab. 3.1).

Unfortunately, purification of the cross-linked constructs resulted in high levels of unspecific co-isolated protein (Figure 3.12 B). Hence, further analysis of potential specific bands below the prominent band was impeded. Additional attempts to improve the purification process, by using higher amounts of cell lysate or by 
double purification using both tags in sequence (data not shown), did not significantly improve the results.

Certainly, isolation methods for low molecular weight bands, where cross-linking products of small ribosomal proteins are generally expected, have to be improved. But in summary, these results demonstrate suitable conditions for the in vivo incorporation of $\mathrm{pBpA}$ into recombinant constructs of Mdm38K.

\section{Tab. 3.1 Results for mass spectrometry}

\begin{tabular}{|ll|}
\hline Protein & Description \\
MukB & Chromosome partition protein \\
EFTu1 & Elongation factor Tu 1 \\
Tig & Trigger factor \\
Odo2 & $\begin{array}{l}\text { Dihydrolipoyllysine-residue succinyltransferase component of 2-oxoglutarate } \\
\text { dehydrogenase complex }\end{array}$ \\
GatZ & D-tagatose-1,6-bisphosphate aldolase subunit \\
AcrA & Multidrug efflux pump subunit \\
RpoC & DNA-directed RNA polymerase subunit beta' \\
RpoB & DNA-directed RNA polymerase subunit beta \\
HrpA & ATP-dependent RNA helicase \\
\hline
\end{tabular}

\subsubsection{C315 of Mdm38 is accessible for maleimide-mediated in vitro} modifications

An approach to minimize unspecific co-isolations is an in vitro setup. Herein, isolated components, ribosomes, and $\mathrm{Mdm} 38 \mathrm{~K}$, are mixed in an equimolar ratio and incubated for binding. Associated proteins can then be cross-linked by different methods.

As an initial approach, C315 was tested for its availability to externally added cysteine specific maleimide modifications (Figure 3.13 A \& B). Because this amino acid represents the only cysteine within the whole Mdm38K-construct and is also localized in the center of the assumed binding cavity (see Figure 3.5), C315 seemed suitable for this approach. Alexa Fluor ${ }^{\circledR} 488 \mathrm{C}_{5}$ Maleimide was used to test availability for modifications, since it enables detection of the cross-linked product by fluorescence. After co-incubation of Alexa Fluor ${ }^{\circledR} 488 \mathrm{C}_{5}$ Maleimide with Mdm38K, samples were separated by SDS-PAGE (Figure 3.13 C). Equal loading was visualized by coomassie staining, whereas successful modification was indicated by an increase in fluorescence. Although the reaction takes place under nondenaturing conditions, results indicate that C315 is available for maleimidemediated modifications. This confirms the accessibility of C315 and are thus in line with the surface exposure of C315 predicted in the published crystal-structure. 
Accordingly, in vitro approaches using externally added cysteine cross-linkers are suitable for the Mdm38K-construct introduced here, but were not performed within this thesis.

A

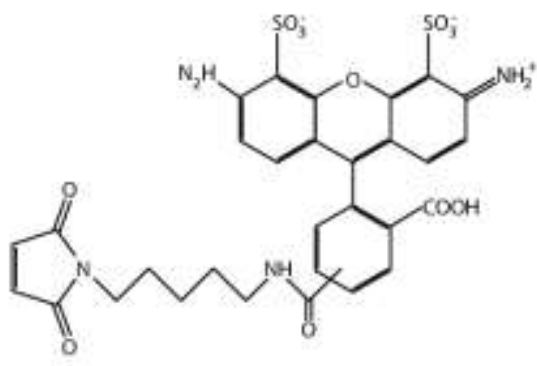

Alexa Fluor 488 C5 Maleimide

C

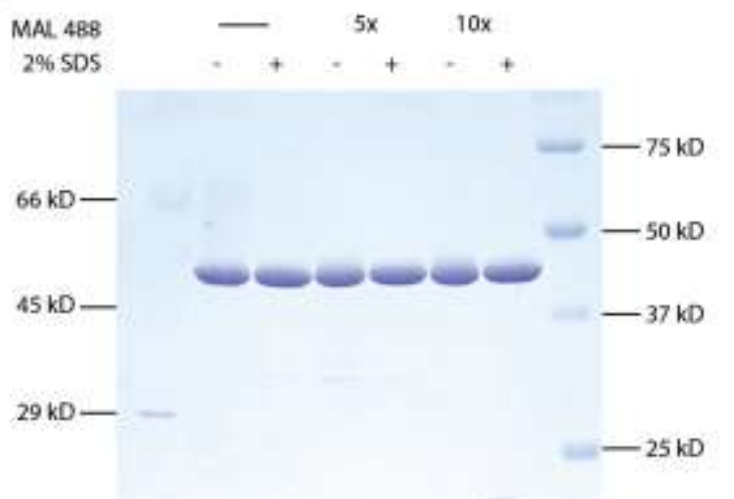

B
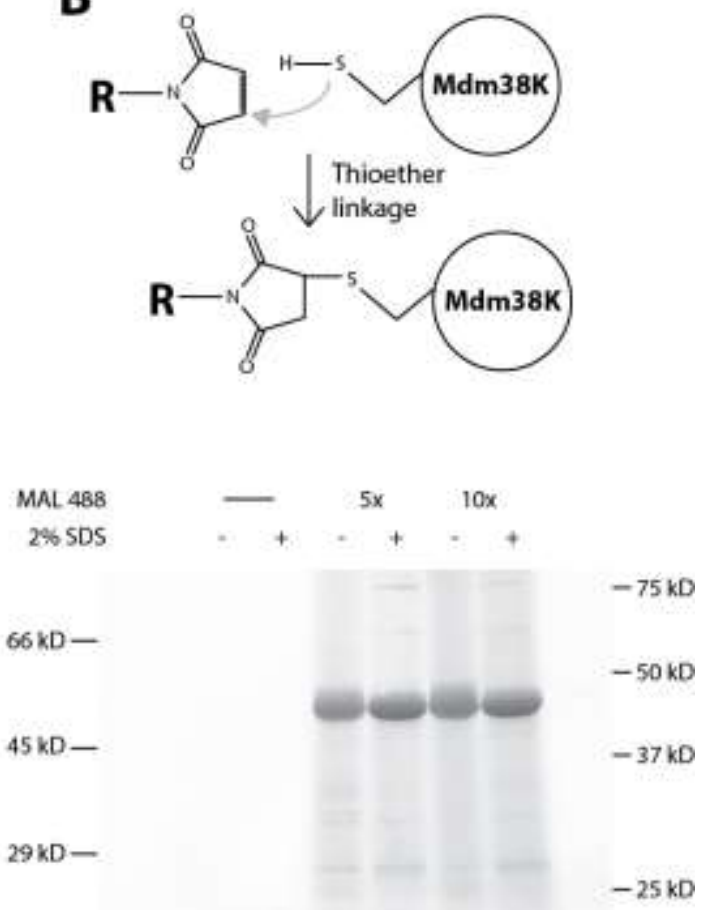

Figure 3.13 Modification of C315 of Mdm38K

A) Cysteine specific fluorescent cross-linker Alexa Fluor ${ }^{\circledR} 488 \quad \mathrm{C}_{5}$ Maleimide (Thermo Fisher Scientific)

B) One step reaction of thioether formation between a maleimide cross-linker and a cysteine (Sahaf et al. 2003)

C) In vitro modifications were performed with purified Mdm38K and Alexa Fluor ${ }^{\circledR}$ $488 \mathrm{C}_{5}$ Maleimide (MAL 488). The maleimide reactant was added in indicated molar excess. Shown are a coomassie stained SDS-gel (left) and a fluorescence scan of a Western Blot using the same samples (right). As positive controls, reactions were simultaneously performed in presence of $2 \%$ SDS. 


\subsection{Overexpression phenotype of Mdm38}

\subsubsection{Suppression and creation of phenotypes by extra-chromosomally expressed Mdm38}

Previous studies focusing on the ribosomal binding domain of Mdm38 made use of different chromosomally expressed truncations of the protein. Specifically, a truncation construct, lacking the amino acids C-terminal from the predicted transmembrane domain (Mdm38 ${ }^{\mathrm{RBD}-\mathrm{ProtA}} /$ aa1-159), exhibits a similar, but slightly more severe, phenotype than the deletion mutant (Lupo et al. 2011). For this reason, we considered the possibility of a dominant effect caused by the truncation. It was therefore tested, whether extra-chromosomal expression of wild-type Mdm38 suppresses the phenotype. The plasmids pRS416 and pRS416 MDM38 (2.1.6) were transformed into Mdm38 ${ }^{\triangle \mathrm{RBD}-\mathrm{ProtA}}$ using $m d m 38 \Delta$ and WT as controls. Suppression of the phenotype was monitored by growth tests (2.2.3). Expression of pRS416 MDM38 reverted the growth phenotype of Mdm38 ${ }^{\Delta R B D-P r o t A}$ and $m d m 38 \Delta$ (Figure 3.14) and so it does not confirm the hypothesis of a dominant effect. Simultaneously, the same experiments were performed using the multicopy vector Yep352. While complementation of the $m d m 38 \Delta$ growth phenotype was rescues by plasmid based expression, Yep352 MDM38, further referred to as the Mdm38 overexpression strain or Mdm380ex, caused reduced growth on nonfermentable medium.

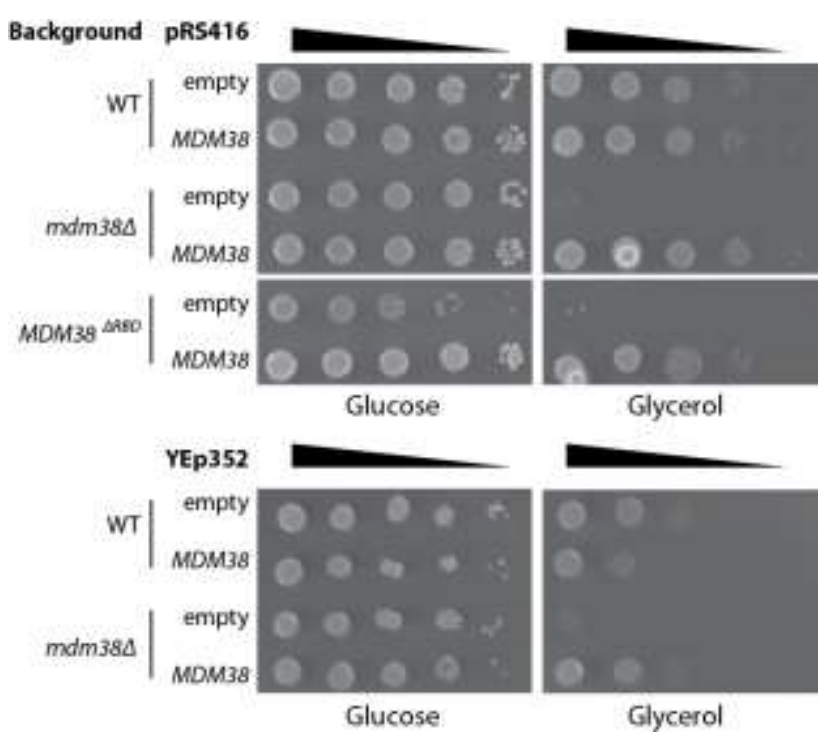

Figure 3.14 Growth test for plasmid containing strains

Shown is a drop dilution assay for yeast strains transformed with pRS416 or Yep352 either empty or with insert (MDM38). Overnight cultures were washed and adjusted to $2 \mathrm{OD}_{600}$ in water. Serial dilutions were spotted on selective agarmedium (SM -Ura) containing either glucose or glycerol as carbon sources. Plates were scanned after 3 days incubation at $30^{\circ} \mathrm{C}$. 


\subsubsection{Overexpression strains resemble the $m d m 38 \Delta$ phenotype on Western}

\section{Blot and Blue Native PAGE}

Since Mdm38 overexpression resulted in this novel phenotype, we performed further studies for characterization. First, the expression level of Mdm38 was determined by Western Blot analysis using fluorescent secondary antibodies (2.1.4 \& 2.4.4). Quantification and comparison of consistent signals indicated a mild $2.3(+/-0.1)$ fold overexpression of Mdm38, compared to wild-type levels.

Furthermore, steady state levels of mitochondrial proteins were determined by Western Blot analysis of whole cell extracts (data not shown) and isolated mitochondria (Figure $3.15 \mathrm{~A}$ ). As a result, $m d m 38 \Delta$ showed reduced amounts for different proteins of complex III (Rip1) and complex IV (Cox1-4, Cox13) of the respiratory chain, whereas all other tested proteins remained unchanged. This is in line with former publications (Frazier et al. 2006). Additionally, proteins affected in the $m d m 38 \Delta$ background were recovered to wild-type levels, as long as Mdm38 is extra-chromosomally expressed from pRS416. On the contrary, additional expression of Mdm38 via pRS416 in wild-type yeast had no detectable influence. In addition, Mdm380Ex exhibits reduced levels of all proteins that also have reported changes in $m d m 38 \Delta$.

Altogether, these results are in line with the growth phenotypes described above. These data support the assumption of disrupted mitochondrial translation and assembly of respiratory chain complexes in Mdm380Ex, which was published earlier for $m d m 38 \Delta$.

Mitochondrial complexes were investigated using Blue Native PAGE (2.4.2). Isolated mitochondria were lysed under non-denaturing conditions with digitonin. Subsequently, solublilized proteins were separated on a gradient polyacrylamide gel and corresponding Western Blots were immunostained for different mitochondrial complexes (Figure $3.15 \mathrm{~B}$ ). On the one hand, it was found that complexes and supercomplexes of the respiratory complexes III and IV exhibit reduced signals. On the other hand, lower molecular weight bands for Cox4 $\left(^{*}\right)$ indicate either an incompletely assembled complex IV or degradation of the respective products. Similarly, $\mathrm{F}_{1} \mathrm{~F}_{0}-\mathrm{ATPase}$ (complex V) also showed slightly reduced amounts of its monomeric (V) and dimeric forms $\left(\mathrm{V}_{2}\right)$ in $\mathrm{Mdm} 38^{\mathrm{OEx}}$ and 
$m d m 38 \Delta$. In a longer exposure, some lower molecular weight signals $\left(^{*}\right)$ became visible, suggesting deficiencies in either assembly or stability of the ATPase. These results are support the published phenotypes of $m d m 38 \Delta$ and suggest that overexpression of Mdm38 results in a similar, but milder, phenotype in $S$. cerevisae.

A)
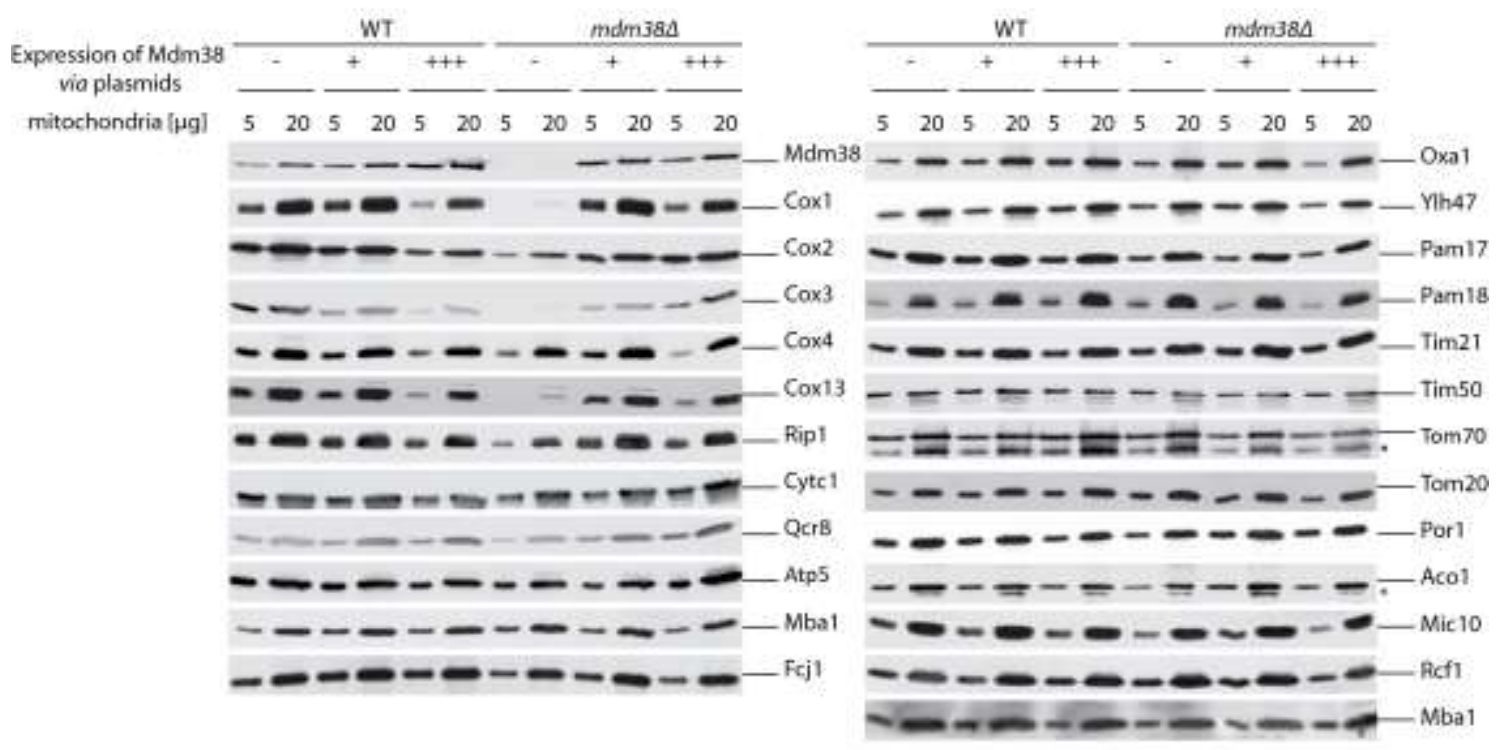

$\begin{array}{lllllllllll}20 & 5 & 20 & 5 & 20 & 5 & 20 & 5 & 20 & 5 & 20\end{array}$

B)

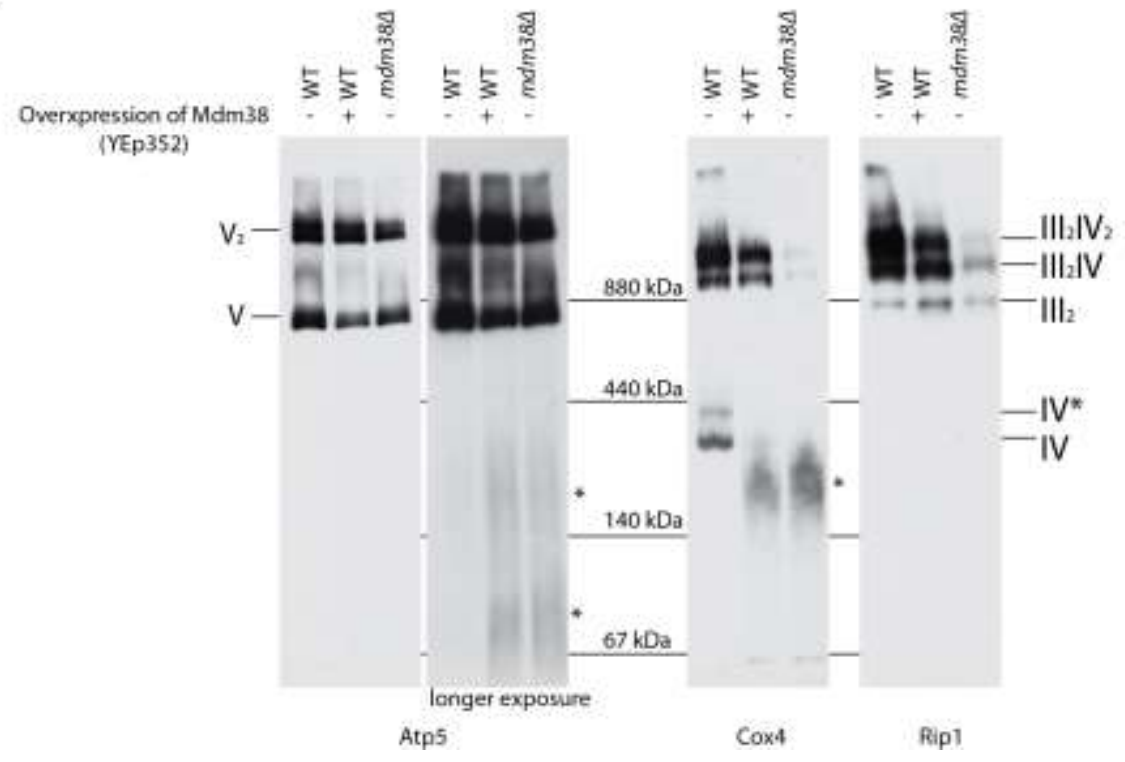


Figure 3.15 Mdm38 overexpressing strains resemble a mild phenotype of mdm38

A) Isolated mitochondria from the indicated strains were lysed in SDS loading buffer and separated by SDS-PAGE. Mdm38 was expressed from pRS416 (+) or Yep352 $(+++)$ using empty Yep352 as a control (-). The corresponding Western Blot was immunostained for the indicated proteins, whereas asterisks indicate bands resulting from cross-reactions of used antibodies.

B) Isolated mitochondria were solubilized with $1 \%$ digitonin and subjected to Blue Native PAGE $(20 \mu \mathrm{g})$. The corresponding Western Blot was stained for different proteins of the respiratory chain complexes (IV \& V) and supercomplexes $\left(\mathrm{III}_{2} \mathrm{IV} \&\right.$ $\mathrm{III}_{2} \mathrm{IV}_{2}$ ) are labeled, whereas $\mathrm{IV}^{*}$ labels an assembly intermediate of complex IV. Single asterisks indicate lower molecular weight signals for Atp5 and Cox4, arisen either from incomplete assembly or degradation of corresponding complexes.

\subsubsection{Mdm38 overexpression does not affect translation}

Reduced amounts of respiratory chain complexes and corresponding proteins could both result from deficiencies in translation and, possibly linked to that, deficiencies in assembly during mitochondrial biogenesis. To clarify both possibilities, further experiments were performed.

Previous publications suggest deficient $\mathrm{K}^{+} / \mathrm{H}^{+}$-homeostasis in $m d m 38 \Delta$ is the main cause of these phenotypes (Froschauer et al. 2005; Nowikovsky et al. 2007). The main argument for this hypothesis is the fact that the artificial $\mathrm{K}^{+} / \mathrm{H}^{+}$-exchanger nigericin mitigates the various phenotypes. Interestingly, further studies showed that defects in mitochondrial translation for $m b a 1 \Delta / m d m 38 \Delta$ double mutants and Mdm38 $\triangle R B D$ truncation were not rescued by the drug (Bauerschmitt et al. 2010; Lupo et al. 2011). Therefore, the authors excluded ion homeostasis as the exclusive cause of these effects.

To this end, Mdm380Ex was investigated for potential suppression of its growth phenotype by application of nigericin. A drop dilution assay was performed on selective media supplemented with $0.5 \mu \mathrm{M}$ nigericin (Figure 3.16). Consistent with previous publications, growth on nigericin-medium rescues growth to wild-type levels for $m d m 38 \Delta$, whereas Mdm38 ${ }^{\circ \mathrm{Ex}}$ is unchanged. Control plates without the drug exhibit characteristic growth phenotypes for $m d m 38 \Delta$ and Mdm380Ex on nonfermentable medium. These results argue that the observed phenotype is at least not exclusively caused by deficiencies in potassium-homeostasis. 


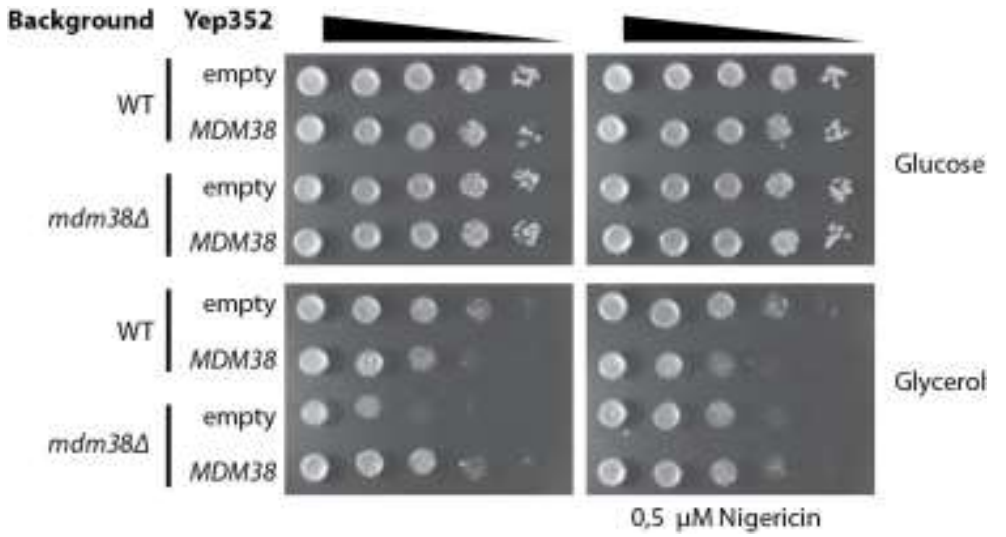

Figure $\quad 3.16 \quad$ Nigericin does not mitigate the growth phenotype

A drop dilution assay was performed for indicated yeast strains transformed with Yep352 derivatives as described for Figure 3.6. Additionally, medium supplied with $0.5 \mu \mathrm{M}$ nigericin was used.

To gain an insight into possible changes in mitochondrial translation by the overexpression of Mdm38, mitochondrial translation products were radioactively labeled with ${ }^{35} \mathrm{~S}$-methionine in living yeast cells (2.4.12). The autoradiogram of the corresponding SDS-gel reveals the known mild reduction of newly synthetized Cox1 and Cytochrome $b$ for $m d m 38 \Delta$. On the contrary, signals for all other tested strains suggest no changes in mitochondrial translation (Figure 3.17).
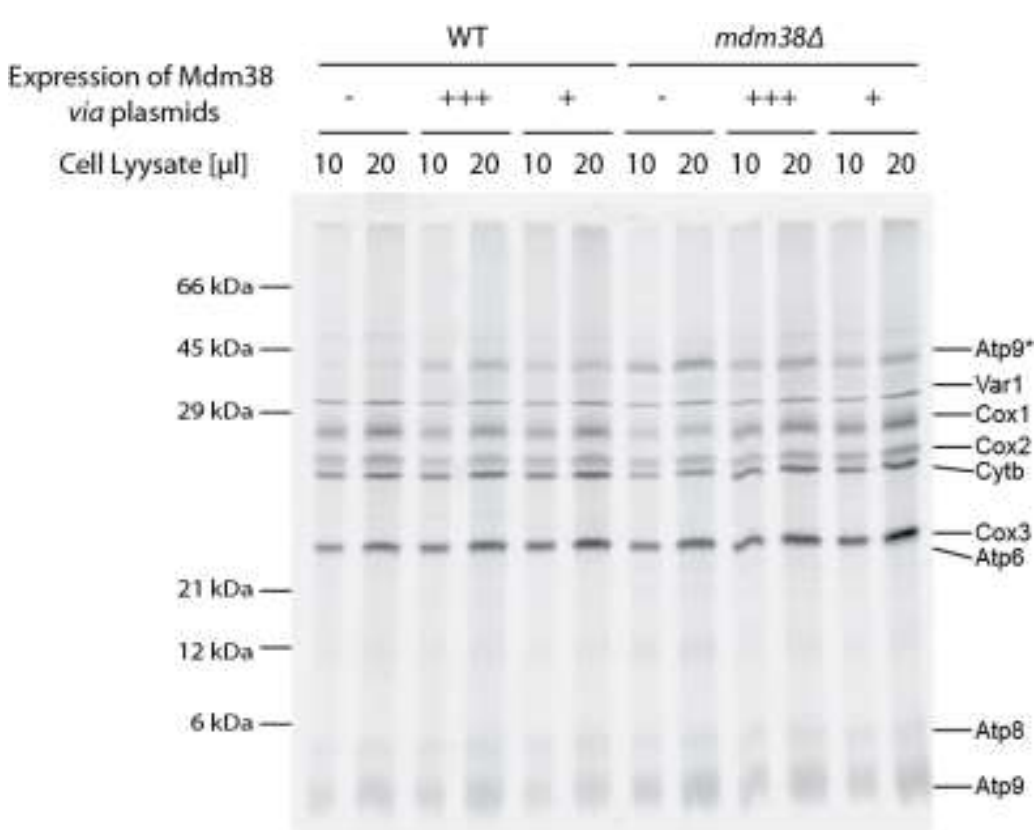

Figure 3.17 In vivo labeled mitochondrial translation products are not affected in Mdm38 OEx

Mitochondrial translation products were labeled with ${ }^{35} \mathrm{~S}$-methionine for 10 min. Proteins were subsequently precipitated with TCA and separated by SDS-PAGE. The dried gel was subjected to digital autoradiography. Expected sizes of mitochondria-encoded proteins are labeled. The asterisk indicates the oligomer of Atp9.

Since reduced protein amounts for $\mathrm{Mdm} 38^{\mathrm{OEx}}$ cannot be explained by dysfunctions in mitochondrial translation, the stability of newly synthetized proteins might be the reason. To this end, in vivo pulse-chase experiments were performed (2.4.13). Cells were incubated at $30{ }^{\circ} \mathrm{C}$ for different chase times after incorporation of ${ }^{35} \mathrm{~S}$ - 
methionine was stopped. Corresponding SDS-gels were dried and subjected to digital autoradiography. Signals for Cox1, Cox2 and Cytochrome $b$ were quantified, internally normalized to Var1 and graphically depicted as percentages of samples at 0 min chase (Figure 3.18). These data demonstrated that the stability of the investigated proteins is not significantly altered within the monitored timeframe. In summary, mild overexpression of Mdm38 causes a phenotype, which, in tendency, resembles the delta mutant but is non-reversible by nigericin. However, neither deficiencies in translation, nor reduced stability could be established as possible causes. Further experiments addressing the assembly of respiratory chain complexes might provide an explanation, but were not conducted within this thesis.

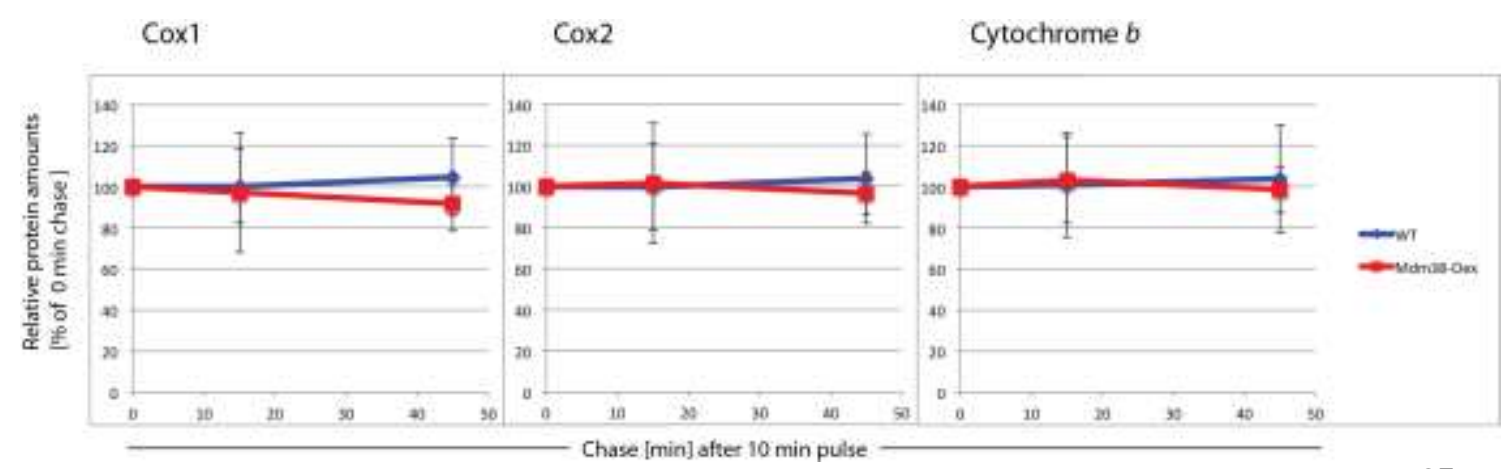

Figure 3.18 Mitochondrial translation products are not destabilized in Mdm38 ${ }^{\circ \mathrm{Ex}}$ Graphs depict results of pulse-chase labeling experiments. Mitochondrial translation products were labeled with ${ }^{35} \mathrm{~S}$-methionine for $10 \mathrm{~min}$ (pulse). Incorporation of radioactive methionine was stopped by the addition of excess unlabeled methionine. Possible degradation was stopped 0,15 and 45 min (chase) on ice. Samples were precipitated, separated by SDS-PAGE and subsequently analyzed by digital autoradiography. Signals for Cox1, Cox2 and Cytochrome $b$ were quantified, internally normalized to Var1 and graphically depicted as percentages of samples with 0 min chase. Error bars indicate the standard deviation of three independent experiments. 


\subsection{Spatially restricted enzyme mediated biotin tagging}

To identify proteins within the close environment of Mdm38, the biotin ligase BirA was used. In principle, BirA fused to a protein of interest, biotinylates other proteins inside a range of approximately $20-30 \mathrm{~nm}$, which then enables isolation and immunodecoration of the biotinylated proteins ((Roux et al. 2012), (Schematically illustrated in Figure 3.19). Accordingly, a fusion construct of Mdm38 with the HA-tagged biotin ligase at its C-terminus (Mdm38 ${ }^{\mathrm{BirA}}$ ) was generated and expressed from the chromosomal locus of MDM38 (Levchenko, 2015 - unpublished).

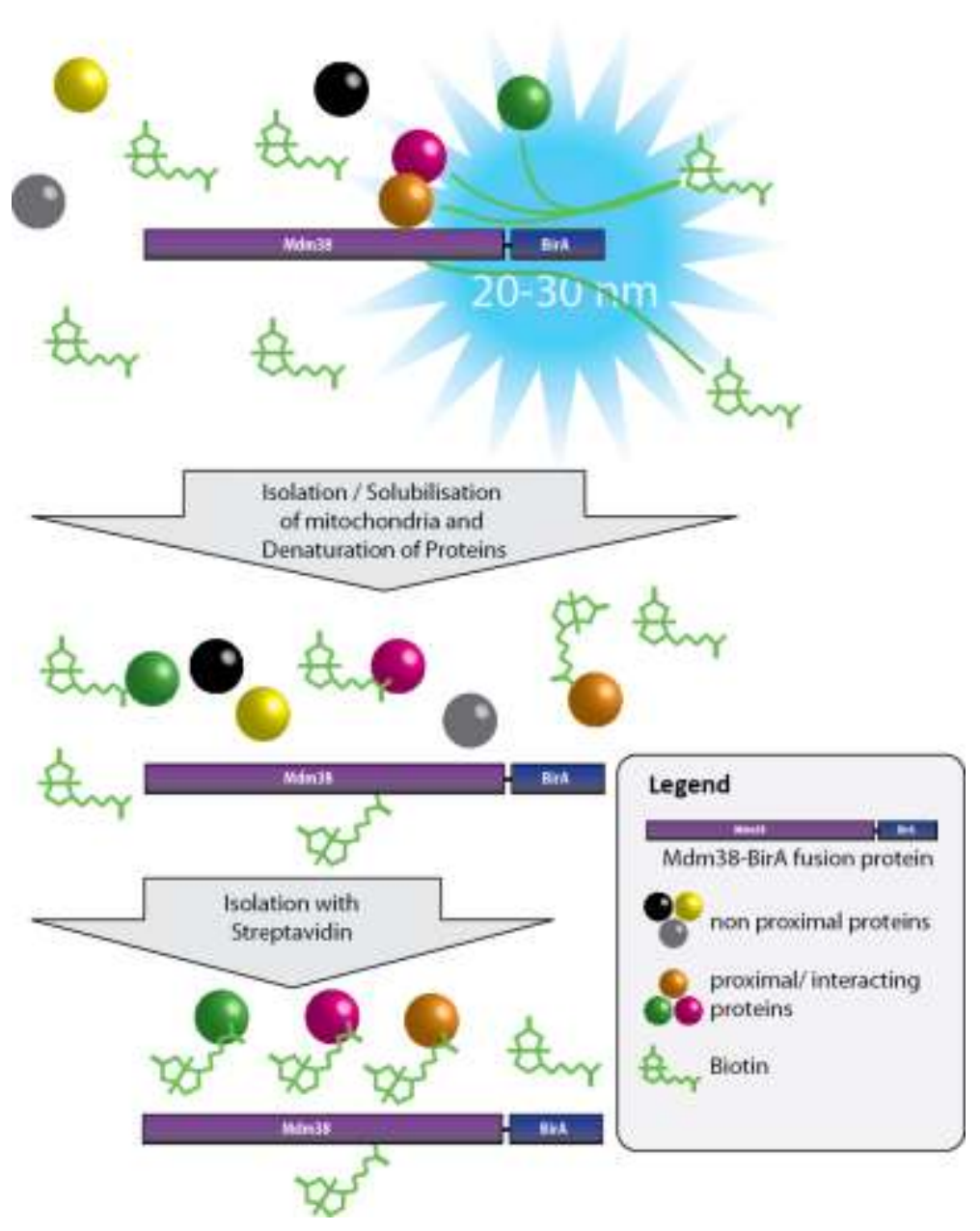

Figure 3.19 Schematic illustration of the biotinylation approach BirA C-terminally fused to Mdm38 auto biotinylates Mdm38 and also proximal proteins in vivo (green lines). Mitochondria were subsequently isolated and lysed under denaturing conditions. Finally biotinylated proteins were isolated with Streptavidin agarose beads.

Figure adapted from Roux et al. 2012

\subsubsection{Characterization of BirA-fusion strains}

To perform investigations with the BirA fusion strains, yeast strains expressing the fusion constructs were firstly examined for their phenotype. The Mdm38 ${ }^{\text {BirA }}$ fusion strain did not show deficiencies in growth or steady state levels of various proteins 
in comparison to the wild-type. It was therefore assumed that Mdm38 ${ }^{\text {BirA }}$ is still functional and suitable for further studies (data not shown).

Since yeast full media is already supplied with biotin, biotinylation should occur under normal growth conditions. In preliminary tests, isolated mitochondria from Mdm38 BirA grown in YPG, were lysed and subsequently affinity purified with Streptavidin agarose beads (2.4.21). The Western Blot of corresponding SDSeluates was stained for biotin using SA-HRP (2.4.5) and exhibited several bands differing in size from the wild-type control, as well as a variety of additional bands (Figure 3.20 A). Furthermore, mitochondria from yeast with an analogous BirA fusion construct of another mitochondrial protein, $\operatorname{Cox} 26$ (Cox26 ${ }^{\mathrm{BirA}}$ ), were used as a control (Levchenko, 2015 - unpublished). In this case, the detected band pattern was different from that of Mdm38 ${ }^{\text {BirA }}$, indicating specific biotinylation products.

The C-terminus of Mdm38 is localized in the mitochondrial matrix, which should also be true for biotinylated proteins. This hypothesis was addressed by determining the submitochondrial localization of biotinylated proteins (2.4.16). Whole mitochondria and mitoplasts (generated by swelling of the mitochondria in order to disrupt the outer membrane) were subjected to PK treatment (Figure 3.20 B). SA- HRP stained bands are only accessible to PK after disruption of all membranes and therefore behave like the matrix protein Mrpl39. In contrast, the OM protein Tom70 is already digested in whole mitochondria, whereas Tim21 (IM) is partially digested in mitoplasts (lower molecular weight band) and completely after sonication.

Based on these results it can be concluded that the majority of bands detected with SA-HRP are located in the mitochondrial matrix and therefore specifically biotinylated by Mdm38 BirA. However, it is worth noting that binding of the Mdm38 specific antibody is prevented for the Mdm38 BirA construct and therefore localization could not be determined directly. 


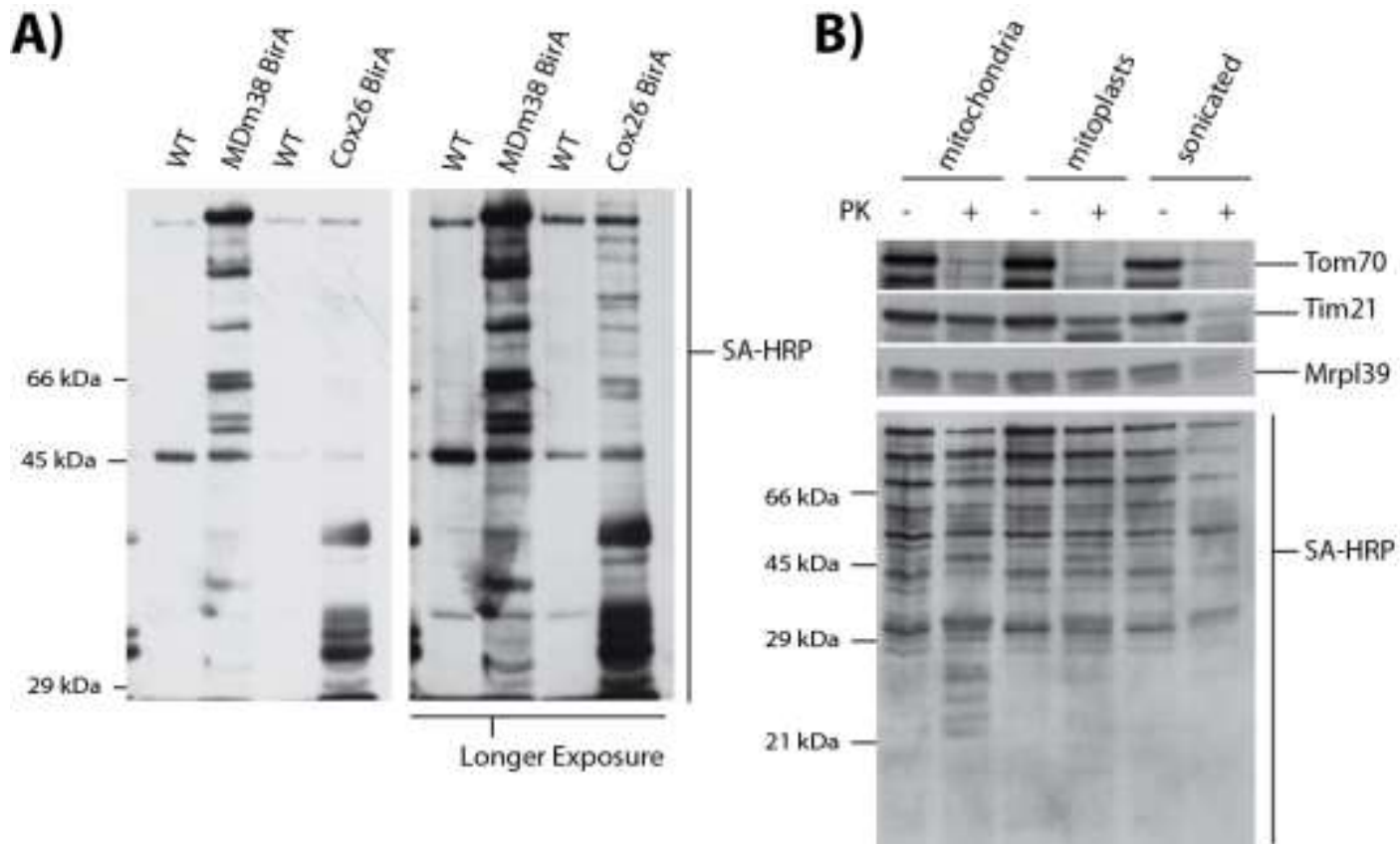

Figure 3.20 Biotinylation by Mdm38 ${ }^{\text {BirA }}$

A) Western Blot of an SDS-PAGE stained against biotin with SA-HRP (short and log exposure). BirA fusion strains of Mdm38 and Cox26 from two different isolations were used, whereas the corresponding wild-type is loaded for each isolation. Samples are SDS-eluates from affinity purification with Streptavidin agarose beads and correspond to $0.5 \mathrm{mg}$ mitochondria.

B) Western Blots of a subcellular localization approach via PK accessibility. Depicted are immunostainings for control proteins within different compartments: Tom70 (OM), Tim21 (IM) and Mrpl39 (matrix). A replica Blot (lower part) was stained against biotin with SA-HRP. PK accessibility is depicted by reduced signal after treatment with $100 \mu \mathrm{g} / \mathrm{ml} \mathrm{PK}(+)$. In mitoplasts Tim21 is partially digested, resulting in a truncation product (lower weight band in corresponding lane).

\subsubsection{Identification of biotinylated proteins}

Since the involvement of Mdm38 in mitochondrial translation and the export of mitochondrially encoded proteins has already been characterized in previous publications (Frazier et al. 2006; Bauerschmitt et al. 2010), newly synthetized proteins were predicted to be candidates for being spatially associated with Mdm38. Therefore, it was hypothesized that mitochondrial translation products are biotinylated by the fused biotin ligase of Mdm38 ${ }^{\mathrm{BirA}}$. To investigate this, an in organello labeling with ${ }^{35} \mathrm{~S}$-methionine (2.4.14) was performed. Herein, the translation buffer (Tab. 2.1) was additionally supplied with $50 \mu \mathrm{M}$ biotin, the substrate for BirA. Mitochondria were then lysed and biotinylated proteins were purified as described above. Samples were separated by SDS-PAGE and analyzed by Western Blot. Digital autoradiography (Figure 3.21) did not reveal any enriched bands above background levels for Mdm38 BirA. Whereas the control strain, 
Cox26 ${ }^{\text {BirA }}$, exhibited an enrichment of three protein bands and therefore confirms the performance of the assay in general.

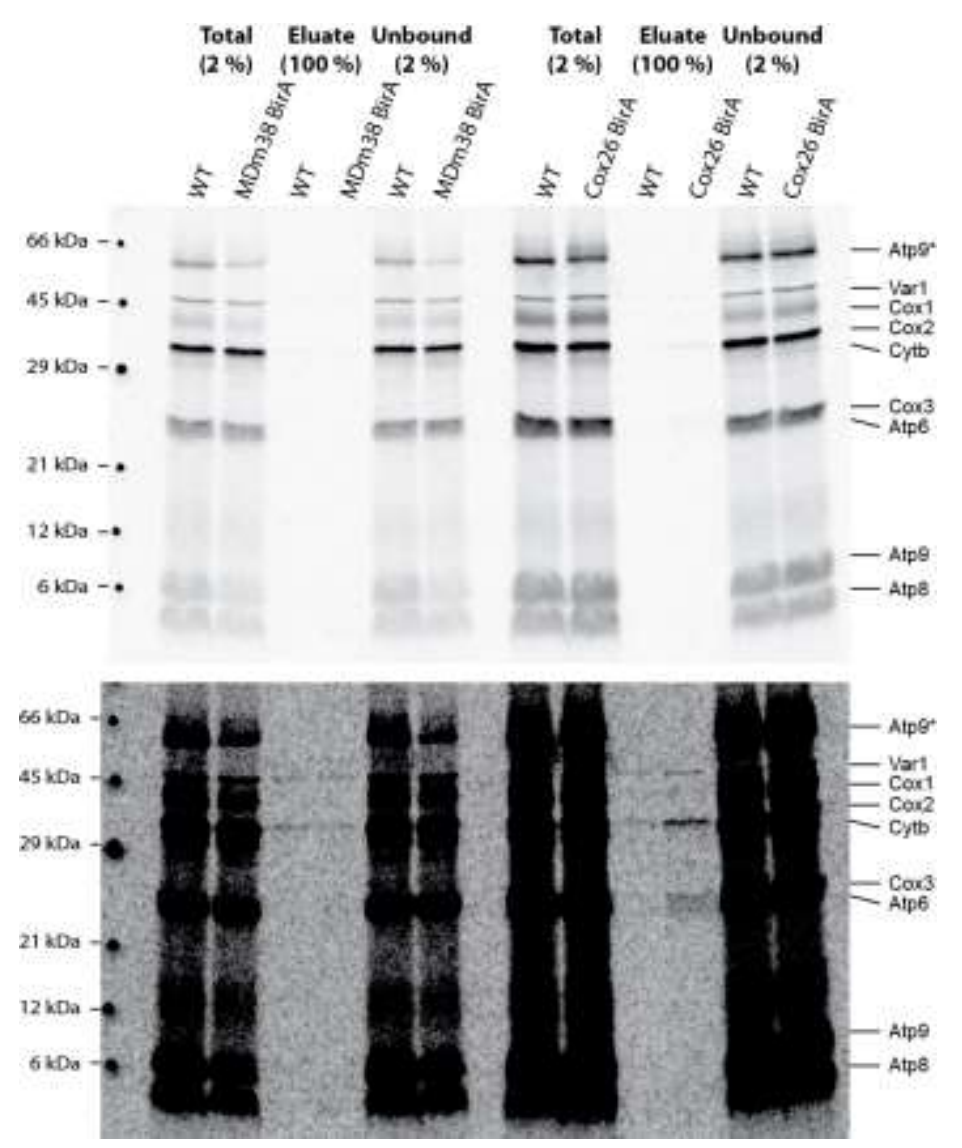

Figure 3.21

Biotinylation of mitochondrially proteins encoded

Autoradiograms of Western Blots from samples, that were achieved after $50 \mathrm{~min}$ in organello labeling of $500 \mu \mathrm{g}$ mitochondria and subsequent isolation with Streptavidin agarose beads. Mitochondria from Cox26BirA expressing yeast were used as a control.

To visualize eluates, the contrast was increased (lower picture). Whereby only for Cox26BirA radioactive bands were enriched corresponding in size to Cox1, Cox2 and Cox3.

Expected sizes of mitochondrially encoded proteins are indicated, whereas the asterisk labels the oligomer of Atp9.

Additionally, Western Blots of Streptavidin isolations from Mdm38BirA were stained for a subset of different mitochondrial proteins (Cox1, Cox2, Cox3, Cox4, Mrpl39, Atp5, Aco1, Tom70, Tim44 - data not shown). However, no detectable amount of the tested proteins was enriched. Thus the identity of the biotinylated proteins remained unclear.

In consequence, further identification was performed using mass spectrometry. For this purpose, $\mathrm{Mdm} 38^{\mathrm{Bir} A}$ isolations from mitochondria were performed as previously described using two Mdm38BirA strains from independent clones. Samples were split and separated on two SDS-gels depicted in Figure 3.22. The Western Blot exhibits the characteristic band pattern of biotinylated proteins (3.4.1). However, only a few bands were visualized by colloidal coomassie staining of the SDS-gel. Since bands of the same size also appeared in the wild types eluates, these bands are likely unspecific co-isolates. Altogether, 5 bands (6-10) were specifically stained for Mdm38 ${ }^{\text {BirA }}$ and therefore cut out and analysed by mass 
spectrometry. Gel pieces from the corresponding positions of eluates from wildtype mitochondria were used as controls (1-5).
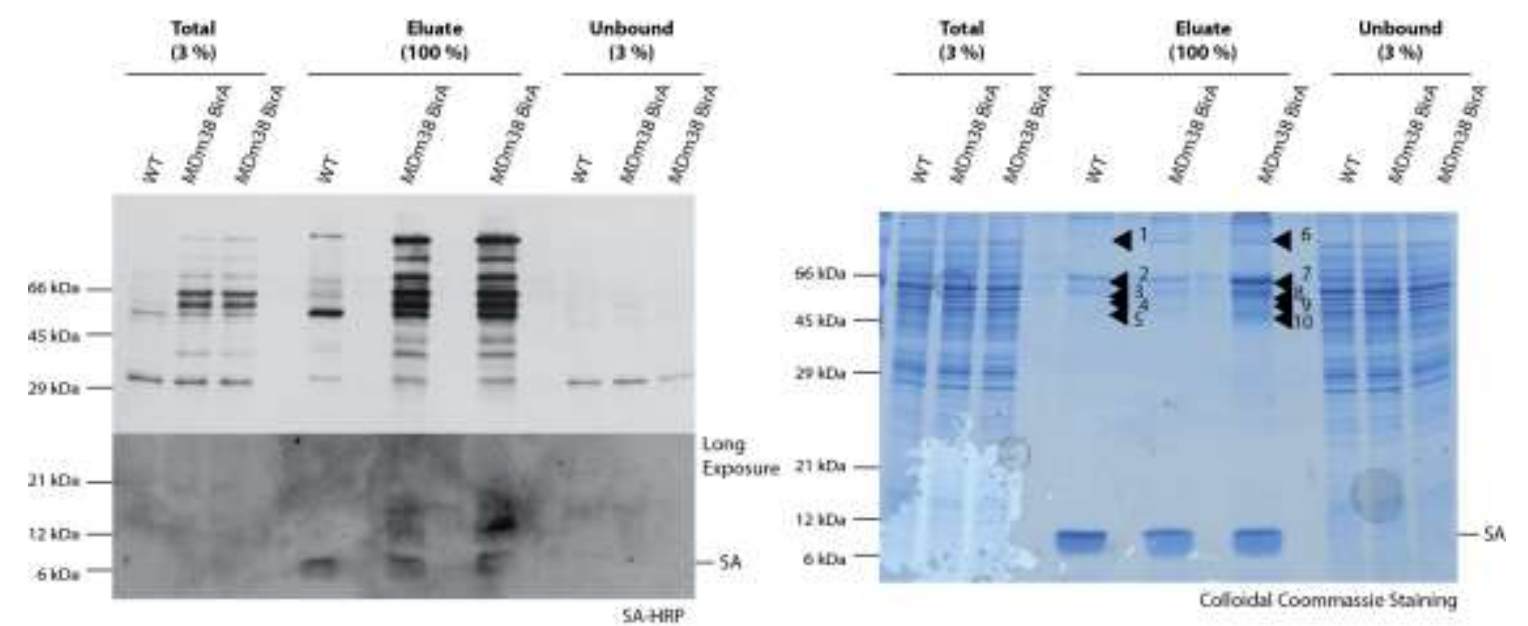

\section{Figure 3.22 Streptavidin isolation for mass spectrometry}

Isolation with Streptavidin agarose from solubilized mitochondria of wild-type or Mdm38 BirA expressing yeast. Samples were split and separated onto two SDS-gels. One gel was blotted (right picture) and probed with SA-HRP, exhibiting several specific bands for Mdm38 ${ }^{\text {BirA }}$ in the total and SDS-Eluates. To detect weaker signals, a longer exposure was used for the lower part of the blot. .

The second gel (left panel) was stained with colloidal coomassie revealing a few bands for all SDS-eluates. Bands specific for Mdm38 ${ }^{\text {BirA }}(6-10)$ and the corresponding running heights of the wild-type (1-5) are indicated by arrowheads

Streptavidin eluted from beads is indicated (SA).

After subtraction of non-mitochondrial proteins and background isolation, which were detected in wild-type eluates, a few proteins were identified. Mdm38 was detected in the most prominent band (6), which indicates an expected autobiotinylation of the construct. Furthermore, according to the working hypothesis, the sample, labelled as band 9, contained the ribosomal $37 \mathrm{~S}$ ribosomal protein Rsm24. Finally, Abf2 (ARS-binding factor 2), a small basic DNA-binding protein was identified in band 9 (Diffley and Stillman 1988). Interestingly, this protein was recently identified in the mitochondrial expressosome complex, termed MIOREX, in which Mdm38 is also involved (Kehrein et al. 2015).

In conclusion, these initial studies confirm previous characterizations and could show, that the approach using BirA is suitable for the characterization of the environment of Mdm38. 


\section{Discussion}

Mdm38 is a nuclear encoded protein localized at the matrix side of the inner mitochondrial membrane. There, the protein is involved in the maintenance of mitochondrial morphology (Dimmer et al. 2002). Further studies also demonstrated a role for Mdm38 in potassium homeostasis suggesting a general effect for mitochondrial function and biogenesis (Nowikovsky et al. 2004; Froschauer et al. 2005; Zotova et al. 2010). In addition, Mdm38 was shown to have a function in mitochondrial translation and interacts with mitochondrial ribosomes, as well as other proteins involved in mitochondrial protein biogenesis (Frazier et al. 2006; Bauerschmitt et al. 2010; Kehrein et al. 2015).

Finally, recent results from Lupo et al. identified the ribosomal binding domain of Mdm38, which shows structural similarity to 14-3-3 proteins. In their studies, Lupo et al. proposed a highly conserved cavity as a potential binding site of this domain consistent with 14-3-3 proteins. Since this ribosomal binding domain was also shown to be essential for Mdm38 function in mitochondrial translation, independent from its role in potassium homeostasis, the primary objective of the present thesis was to further characterize this domain and discover potential binding partners.

\subsection{Binding to $E$. coli ribosomes}

In vitro binding studies of recombinantly expressed constructs containing the ribosomal binding domain (RBD), with GSH-Sepharose already confirmed an interaction with yeast mitochondrial ribosomes \{Lupo:2011iw\}. Using the same constructs as Lupo et al., the present thesis demonstrates that E. coli ribosomes are also enriched in the isolations of the respective Mdm38 constructs (Figure 3.2). In order to gain further insights into binding characteristics, the dependence of binding on ionic strength was tested. Data provided here indicate a salt-sensitive binding to the ribosome, since co-isolation was almost completely lost after washing of the beads with a high-salt buffer (Figure 3.3). Thus, these observations are consistent with former publications for Mdm38 in yeast, that describe a loss of binding to mitochondrial ribosomes under high-salt conditions (Frazier et al. 2006; 
Kehrein et al. 2015).

In this context, the observed salt dependent binding affinity can be correlated to different published features relating to the structural identity of the ribosomal binding domain (Mdm38K) (Lupo et al. 2011):

(a) The fact that the primary interaction between other 14-3-3 proteins and their ligands is mainly mediated by the formation of numerous polar contacts is consistent with the observed salt-sensitivity (Yaffe et al. 1997; Rittinger et al. 1999). However, the predicted binding pocket, within a highly conserved area of the Mdm38 RBD, is not capable of the binding properties described in latter publications, because here binding involves phosphoserine/-threonine containing motifs within the ligand. Thus, the mainly hydrophobic surface in the cavity argues against this reason (Lupo et al. 2011). Therefore, another phosphorylationindependent mode of binding, as suggested by Ottmann et al., is possible. This form of binding is mainly driven by hydrophobic interactions via leucine side chains, which are conserved in Mdm38 and other 14-3-3 proteins (Ottmann et al. 2007).

(b) Another common feature of 14-3-3 proteins is a homo- or hetero-association of monomers, leading to the formation of stable dimers of a clamp-like shape (Gardino et al. 2006). In this case, interaction is achieved by multiple hydrophobic and polar contacts, as well as several salt bridges depending on the protein involved. Correspondingly, Zotova et al. (2010) revealed a self-dimerization of Mdm38 in mitochondria; a finding, that can be further correlated to similar results for the human homologue, Letm1, by Hasegawa et al (2007). Consequently, these observations could also represent a feature that depends on the ionic environment and thus, might explain the loss of binding under high salt conditions presented in this work. Cautiously, comparability in this regard is only scarce, because this multipoint interaction described by Gardino et al. involves residues of the first four $\alpha$-helices of 14-3-3 proteins, whereas helices 1 and 2 are not present in the Mdm38 domain (Lupo et al. 2011). Therefore, dimerization is expected to take place by yet uncharacterized but probably also salt-dependent mechanisms. Nevertheless, it should be noted that although dimerization is a widespread feature in the protein family, it is not functionally essential for all 14-3-3 proteins. A variety of 14-3-3 proteins are described, whose functions were shown to be independent from dimerization (Sluchanko et al. 2011). Furthermore, a spliced variant of the human 
14-3-3e protein, lacking the first 22 residues responsible for 14-3-3 dimerization, still fulfills the function of the normally occurring dimer (Han et al. 2010). To address whether the self-dimerization of Mdm38 shown by Zotova et al. is essentially correlated to ribosomal binding, dimerization of recombinant constructs needs to be further investigated. Dimerization can be shown by nondenaturing separation methods (e.g. Blue Native-PAGE, Gelfiltration) or the coisolation of differently tagged constructs, either co-expressed in vivo or mixed in vitro. The possibility of hetero-dimerization of Mdm38 with another 14-3-3 protein is only speculative, since until now, no other protein of this family has been described in yeast mitochondria.

(c) Finally, salt-dependent binding of Mdm38K to the ribosome could also take place at charged patches, distinct from the investigated cavity, or maybe also at the positively charged rim of the cavity, which is suggestive of an interaction site for negatively charged ribosomal proteins or RNA. Cross-linking approaches, such as that established for the cavity in the present thesis, are an option for proving this hypothesis. In this context, the influence of modified charges at respective positions, via mutagenesis PCR, could provide further insights into binding characteristics. An approach to test for RNA binding and the simultaneous identification of respective RNA was described by Hieronymus \& Silver (2003). Here, RNA is isolated from immunopreciptations and used as a template for reverse transcription, followed by quantitative PCR. For further identification, microarray analysis, or sequencing of corresponding cDNA, can be performed (Hieronymus and Silver 2003; Gilbert et al. 2004).

In addition, a recent publication concerning yeast mitochondrial ribosomes discovered large complexes termed MIOREX, which contain Mdm38, along with other mitochondrial translation components, as well as factors required for transcription (Kehrein et al. 2015). It is therefore, tempting to speculate that the majority of interactions within MIOREX are indirect. For example, Pet309 (see 1.3.2), a protein that interacts with Mdm38, can in contrast to Mdm38 still be purified with mitochondrial ribosomes under high-salt conditions (Kehrein et al. 2015). Thus, Pet309 might represent a linking protein that is not a ribosomal component itself. Interestingly, isolated E. coli ribosomes are capable of binding 
GSH-Sepharose with immobilized RBD containing constructs after the previous removal of ribosomes via high-salt washing. In summary, the demonstrated in vitro binding of purified components is a strong hint for a direct interaction of Mdm38 with ribosomes.

\subsection{In vivo photo cross-linking with $\mathrm{pBpA}$}

The crystal structure of the ribosomal binding domain, Mdm38K, is a powerful basis for further studies concerning potential binding partners. A powerful assay to identify proteins in close proximity to regions of interest and to determine the binding characteristics of $\mathrm{Mdm} 38 \mathrm{~K}$, potentially down to the level of involved amino acids, is in vivo photo cross-linking. This approach was established for $E$. coli via the incorporation of the non-natural amino acid pBpA (Farrell et al. 2005). Therefore, one part of this current work focused on the establishment of this method.

Due to the relatively short cross-linking range of pBpA ( $6.5 \AA$ (Banta et al. 2014)), results are very specific. However, this also means, that pBpA-incorporation needs to occur basically exactly at the site of interaction. In fact, conserved amino acids are most likely to be functional and thus, also required for interactions. Therefore, amino acids were chosen for exchanges that are not only highly conserved among tested species, but also surface-exposed in the center of the predicted binding cavity (Lupo et al. 2011). Prior to pBpA-incorporation, the functional relevance of the chosen amino acid residues were tested in yeast. Surprisingly, single exchanges of all positions by alanine were still functional, as extra chromosomal expression of the respective Mdm38-variants rescued the phenotypes of the delta mutant (Figure 3.6). Therefore, all six amino acids were assumed suitable for exchanges by pBpA.

Preliminary expression tests for constructs containing an amber stop for pBpA incorporation were performed in E. coli using pSUMO-plasmids (Figure 3.7). The results obtained indicate an efficient termination at the intended position, and that read-through or restart of translation after the stop-codon are, if happening at all, only rare events.

To enable efficient suppression of the stop codon through incorporation of $\mathrm{pBpA}$, expression has to be relatively slow (personal communication with Prof. Georg Koch, Freiburg). On this account, two expression-vectors were tested in this thesis: 
arabinose-inducible pBAD24 and IPTG-inducible pTRc99a. Interestingly, only weak translation could be demonstrated for full-length Mdm38K variants using both vectors and for mutagenized domains for only using pTRc99a only. Moreover, results for the pTRc99a-derivatives indicate variations in expression-efficiency that deviate from that of the full-length construct. In particular, the Western Blot signals for the G319-, L330-, and C315-exchanges were remarkably reduced (see Figure 3.9.). These observations indicate a negative effect on the translation or transcription of their respective products, maybe due to secondary structures facilitated by the modifications. In this context, it should be noted that availability of $\mathrm{pBpA}$, and thus potential suppression of the stop codon, did not circumvent these translation problems. This in turn, points to mRNA and/or transcriptionlevel as a cause for these effects.

Successful pBpA-incorporation was only shown for the other three constructs, with TAG-codons at positions M320, G319, and W337. Although expression levels without $\mathrm{pBpA}$ for all three constructs were similar, $\mathrm{pBpA}$ incorporation, indicated by the amount of full-length protein, was different. This deviation can possibly be explained by the findings of Miller et al., that indicate an influence of the base sequence on the 3' side of the stop-codon (Miller and Albertini 1983). More precisely, they found that if the following codon on the respective mRNA begins with cytosine (C) or uracil (U), suppression of the amber-stop is usually low. Correspondingly, the weakest incorporation into $\mathrm{Mdm} 38 \mathrm{~K}$ was revealed for the W337-exchange (Figure 3.10), which is indeed followed by $\mathrm{C}$ in the next codon. In consequence, an option to circumvent this problem is the additional mutagenesis of the respective $C$ to $A$ or $G$, but this was not tested within this thesis.

In summary, pBpA-specific cross-linking bands were exclusively detected for Mdm38K with the best expression and incorporation occurring in Mdm38KM320pBpA. Unfortunately, purification of this construct after pBpA incorporation and cross-linking contained a high background of co-purified proteins also visible in the pBpA-lacking non-mutagenized controls (Figure 3.12). For this reason, only the most prominent band was cut out and analyzed by mass spectrometry. In contrast with the working hypothesis, no ribosomal protein was identified using this approach (Tab. 3.1). However, trigger factor (Tig) represents a ribosome associated protein, which was shown to bind the 50S subunit of the bacterial 
ribosome (Merz et al. 2008; Hoffmann et al. 2010). Specifically, it functions in the folding of the nascent polypeptide chain and might also be involved in the posttranslational stabilization of other proteins (Martinez-Hackert and Hendrickson 2009). At this point it is not clear why Tig appears in the cross-linked band. On one hand this might be because of its localization at the ribosomal exit tunnel (RET), at the site where Mdm38 was also proposed (Bauerschmitt et al. 2010; Lupo et al. 2011). On the other hand, its chaperone role might indicate a rather unspecific association to the construct. However, the size of Tig, of approximately $48.2 \mathrm{kDa}$, al least contradicts the possibility of a dimeric crosslinked product, since the Mdm38K-construct is about $41.6 \mathrm{kDa}$ and the analyzed band ran between 150-200 kDa (Figure 3.12).

The same argument applies to EF-Tu (Elongation Factor - Thermo unstable), which was also discovered in the mass spectrometry data. At approximately $43.3 \mathrm{kDa}$, this protein has a comparable size to Tig. Furthermore, EF-Tu also plays a role as a chaperone that facilitates protein folding (Caldas et al. 1998), in addition to its function in translation (see 1.3).

Further, mass spectrometry data identified three more proteins, which also fit in size to a cross-linked product with Mdm38K and comply with the observed running-behavior of the analyzed band. Interestingly, these proteins are associated with steps before translation and include the two subunits of the DNA-directed RNA-polymerases, RpoB (150 kDa) and RpoC (155 kDa) (Ebright 2000), and the RNA-helicase HrpA (150 kDa) (Moriya et al. 1995; Salman-Dilgimen et al. 2013). These findings can be paralleled with studies of Kehrein et al. (2015) in yeast that identified Mdm38 in a large MIOREX-complex, which also contains components for transcription and RNA-processing. However, given the fact that mitochondria only contain a single RNA-polymerase of an unrelated family (Kelly and Lehman 1986; Cermakian et al. 1996), potential binding to E. coli RpoB and RpoC is unlikely to represent a conserved feature of the organelle. In contrast, HrpA belongs to a ubiquitously expressed protein family of DEAD-box proteins (Salman-Dilgimen et al. 2013). Findings of Salaman-Dilgimen et al. suggest a function in RNA-processing for HrpA and its association to the ribosome. Thus, results presented in this thesis correspond to Mdm38 binding to Pet309 (Krause et al. 2004), a protein that is also associated with the ribosome and binds mRNA (Manthey and McEwen 1995; 
Tavares-Carreón et al. 2008). Implicitly, this might suggest a role for Mdm38 in mRNA recruitment, that was proposed earlier by Bauerschmitt et al..

Another four proteins were identified via mass spectrometry within the cut band: MukB, GatZ, AcrA and Odo2. Of those, only the latter has a homologue in yeast mitochondria, Kgd2. However, findings by Repetto et al. for Kgd2, suggest it is a part of the trimeric $\alpha$-ketoglutarate dehydrogenase complex (Repetto and Tzagoloff 1990) and thus is rather unrelated to the currently documented environment of Mdm38 (Kehrein et al. 2015). For these reasons, those four proteins were considered to be unspecific co-isolations and were not further pursued.

Finally it should be mentioned, that it is expected that the performed overall photo-crosslinking was specific for $\mathrm{pBpA}$ and not potentially facilitated by ribosomal or messenger RNA, since the filter used during UV-exposure excludes wavelengths below $300 \mathrm{~nm}$. Correspondingly, pBpA cross-links at a UV-irradiation range of 350-370 nm (Dormán and Prestwich 1994), whereas RNA-cross-linking needs irradiation around $260 \mathrm{~nm}$ (Greenberg 1979).

Because of the highly abundance of background bands for isolations from the pBpA assay, it is reasonable to include a negative control with a construct lacking pBpA. Thus, whole isolations can be analyzed by quantitative mass spectrometry and proteins that were found to form cross-links independently of pBpa can be discounted.

Other options to reduce background reactions involve an in vitro approach (see 3.2.5). For example, isolated constructs with either in vivo incorporated $\mathrm{pBpA}$, or with a bifunctional cross-linker bound after purification, could be used as described by Leitner et al. (Leitner et al. 2010). Using this approach, it was shown that $\mathrm{C} 315$ of Mdm38K is available for maleimide cross-linkers (Figure 3.13).

In addition to cross-linking, another method for in vitro investigation of binding characteristics is the use of fluorescence resonance energy transfer (FRET) measurements (Stryer 1978). This approach exploits the fact that excitation of a donor fluorophore directly attached to a biological sample may result in energy transfer to a nearby (20-80 ̊) acceptor fluorophore on another sample. Interestingly, this approach not only allows the determination of distance and orientation of both fluorophores (Stryer 1978; Remedios and Moens 1995), but 
also enables the determination of transient states (e.g. at translating ribosomes) using stopped flow experiments (Milon et al. 2007). Consequently, using Mdm38K labeled with Alexa Fluor ${ }^{\circledR} 488$ C5 Maleimide, as documented in this thesis (see 3.2.5) could provide a suitable tool for experiments in the future using compatibly labeled ribosomes.

\subsection{Mdm38 neighboring proteins in S. cerevisiae}

In this thesis, the protein environment of Mdm38 was further addressed using an in vivo approach in yeast. As described by Roux et al., a construct with a Cterminally fused biotin ligase, BirA, was used to label proteins within its proximity (Roux et al. 2012). Obtained results revealed a specific band pattern on Western Blot, suggesting the biotinylation of manly matrix-localized proteins. Moreover, preliminary mass spectrometry analyses identified the $37 \mathrm{~S}$ ribosomal protein Rsm24 and the DNA-binding protein Abf2, which is involved in transcription (Diffley and Stillman 1988). These results support the findings of Kehrein et al., as they implicate Mdm38 in transcription, as well as in translation.

A larger overview of Mdm38 interaction partners could be further assessed by quantitave mass spectrometry using whole eluates, as discussed already for pBpAcontaining constructs. Unfortunately, the biotinylation range of BirA (approximately 20-30 $\mathrm{nm}$ as estimated by Roux et al.) aggravates more specific predictions of the localization, since it already exceeds the ribosomal diameter.

However, there are options to narrow results to interactions specific for the ribosome binding domain. In one approach, BirA can be fused to different Cterminal truncations of $\mathrm{Mdm} 38$, as were previously used for ProteinA-isolation experiments by Lupo et al..

Surprisingly, the findings presented here demonstrate that biotinylation via BirA can be also used to isolate newly synthesized proteins from mitochondrial ribosomes. Although biotinylation was proposed as a slow reaction of about $24 \mathrm{~h}$ by Roux et al., translation-products could be isolated with Streptavidin specifically from Cox26-BirA mitochondria after only a $45 \mathrm{~min}$ labeling reaction. In conclusion, this suggests the biotinylation kinetics are suitable for analysis of interactions in shorter time frames per se. However, no isolation of newly synthesized mitochondrial proteins could be detected for Mdm38 BirA. Since Frazier et al. demonstrated an interaction with Mdm38 ProtA, the findings of this thesis might 
indicate a more indirect interaction. In addition, it cannot be excluded that the interactions of Mdm38 BirA with newly synthesized mitochondrial proteins are too transient and therefore not suitable for this method.

In this context, an option to improve kinetics was introduced by Rhee et al. (2013). There, the group made use of an engineered ascorbate peroxidase (APEX) that can also be fused to the protein of interest. APEX oxidizes biotin-phenol in an $\mathrm{H}_{2} \mathrm{O}_{2}$ dependent reaction. Resulting phenoxyl radicals are then able to tag proteins in proximity in less than one minute, which is even more beneficial for these investigations (Rhee et al. 2013).

\subsection{Overexpression of Mdm38 induces a $\mathrm{mdm} 38 \Delta$ like phenotype}

An additional finding of this thesis was the characterization of a novel phenotype caused by mild overexpression of Mdm38 in yeast. In tendency, the overexpression strain (Mdm38 ${ }^{0 \mathrm{ex}}$ ) has a phenotype resembling that of the deletion mutant (3.3.2). This means, deficient growth on nonfermentable media and reduced steady state levels of different respiratory chain proteins. There are also disruptions in the formation of complexes III, IV and V and levels of corresponding supercomplexes are reduced.

Overall, these results can be likened to several observations for human Letm1. Piao et al. reported that overexpression of Letm1 induces necrotic cell death in HeLa cells by the inhibition of mitochondrial biogenesis and ATP production (Piao et al. 2009). This is further supported by Hwang and coworkers, who observed apoptosis of lung cancer cells during Letm1 overexpression, which was matched by decreased mitochondrial biogenesis (Hwang et al. 2010).

An explanation for these findings can be based on the notion that disturbing the balance of translational activators can interfere with respiratory chain biogenesis. Along this line, Fiori et al. showed that overexpression of Pet111, a translational activator of Cox2, surprisingly lead to lower steady state levels of Cox1. This could be correlated to compromised assembly of complex IV, leading to subunit degradation (Fiori et al. 2005). Moreover, these data are in agreement with previous findings showing that overexpression of other translational activator proteins for Cox3 (Pet54, Pet122 and Pet494) or cytochrome $b$ (Cbs2) also negatively affect respiration in general (Wiesenberger et al. 1995; Tzschoppe et al. 2000). Thus, an elevated amount of Mdm38 can possibly be linked to an imbalance 
in the recruitment of different factors, since a physical interaction between Mdm38 and the translational activators Pet309 and Mss51 has already been shown by Bauerschmitt et al.. However, since no significant changes in mitochondrial translation were observed for $M d m 38^{0 e x}$, data provided here argue against an effect caused by deficient mitochondrial biogenesis (Figure 3.17). It was therefore concluded that the reduced levels of mitochondrially encoded proteins may be caused by the steps immediately following translation. Therefore, it was important to determine whether protein stability is compromised in Mdm380ex. Pulse-chase experiments were performed, but did not reveal significant deviations from wildtype yeast. In contrast, strains deficient in respiratory chain assembly exhibit severely reduced stability of mitochondrially encoded proteins within even shorter timeframes. For example, the stability of Cox2 is compromised in yeast harboring a deletion of COX14, which encodes a protein involved in Cox1-expression (Barrientos et al. 2004).

To further investigate the effect of Mdm38 overexpression on respiratory chain assembly, one option is to follow the assembly of radiolabeled mitochondrial translation products into their respective complexes. This was performed in studies by Gruschke et al. using 2-D-analysis which depicts the assembly of cytochrome $b$ into complexes with timely resolution (Gruschke et al. 2012).

Alternatively, dysfunctions resulting from the overexpression of Mdm38 might also be caused by its role in ion-homeostasis. However, data provided here demonstrate that the $\mathrm{Mdm} 38^{0 \mathrm{ex}}$ growth phenotype cannot be mitigated by the addition of the artificial ion-channel, nigericin (Figure 3.16). Therefore, it was concluded that reduced growth on glycerol could not essentially be attributed to deficiencies in $\mathrm{K}^{+} / \mathrm{H}^{+}$-exchange. This notion correlates with studies by WaldeckWeiermair et al., where overexpression of Letm 1 had no effect on $\mathrm{Ca}^{2+}$-exchange, whereas its depletion reduces $\mathrm{Ca}^{2+}$ transport (Waldeck-Weiermair et al. 2011). In contrast, Hasegawa et al. revealed mitochondrial contraction and cristae condensation resulting from Letm1 overexpression. These latter observations represent the reverse effect of a knock down of Letm 1 and thus, might be due to elevated ion-channeling activity. However additional distinct reasons for morphologic aberrations are possible. Accordingly, Tamai et al. indicated that Letm 1 depletion not only leads to swollen, but also elongated mitochondria. These 
observations cannot be simply explained by an elevated osmotic pressure and thus, an additional function in the maintenance of mitochondrial morphology was proposed for Letm1 (Tamai et al. 2008).

As an additional line of evidence, approaches used for studies in Trypanosoma brucei evansi by Hashimi et al. might be adapted to the yeast system. In their studies, the group phenocopied a Letm1 knockdown using the ionophore valinomycin (Hashimi et al. 2013). Valinomycin facilitates the transport of potassium-ions across lipid membranes along an electrochemical gradient and can thus be seen here as an antagonist of nigericin. In consequence, mitochondrial swelling, induced by valinomycin, is prevented after pretreatment of T. brucei evansi with nigericin (Hashimi et al. 2013). Therefore, an application of valinomycin should counteract an effect of elevated $\mathrm{K}^{+} / \mathrm{H}^{+}$-exchange, which might be caused by Mdm380ex. However, the reverse effect by nigericin, which should further enhance a phenotype caused by increased $\mathrm{K}^{+} / \mathrm{H}^{+}$-exchange, was not observed for Mdm380ex during these studies (Figure 3.16).

To this end, the Mdm38 ${ }^{\circ e x}$ phenotypes reported here are likely to be independent from Mdm38 function in ion homeostasis, although possible interrelations cannot be excluded. Therefore, overexpression of Mdm38 might affect the posttranslational roles of the protein that were previously proposed by Bauerschmitt et al.. 


\subsection{Summary}

The data presented in this thesis demonstrate that binding of Mdm38 to ribosomes has conserved characteristics in yeast and E. coli. To gain further insights into potential binding partners, an in vivo photo cross-linking assay was established. Here, preliminary mass spectrometry analysis of a specific cross-linked product revealed proteins involved in pre- and post-translational steps. Nevertheless, further studies are required to improve purifications and to enable the analysis of lower molecular weight proteins.

Further, it was shown that a fusion of the biotin ligase, BirA, to Mdm38 can be used as a tool to identify proteins in the proximity of Mdm38 in yeast. The comparison of present findings to those from previous studies implies a correlation between transcription and translation involving Mdm38.

Finally, this thesis demonstrates a novel overexpression phenotype for Mdm38, which resembles characteristics of the MDM38 deletion mutant. Data presented here argue against an effect attributed to the proteins role in ion-homeostasis, but rather suggest an influence on post-translational processes, causing the respective phenotypes. However, further experiments are required to address possible effects of Mdm38-overexpression on the assembly of respiratory chain complexes. 


\section{References}

Amunts A, Brown A, Bai X-C, et al (2014) Structure of the yeast mitochondrial large ribosomal subunit. Science 343:1485-1489. doi: 10.1126/science.1249410

Arnold I, Pfeiffer K, Neupert W, et al (1998) Yeast mitochondrial F1F0-ATP synthase exists as a dimer: identification of three dimer-specific subunits. The EMBO Journal 17:7170-7178. doi: 10.1093/emboj/17.24.7170

Atkinson GC, Kuzmenko A, Kamenski P, et al (2012) Evolutionary and genetic analyses of mitochondrial translation initiation factors identify the missing mitochondrial IF3 in S. cerevisiae. Nucleic Acids Res 40:6122-6134. doi: $10.1093 /$ nar/gks 272

Banta AB, Cuff ME, Lin H, et al (2014) Structure of the RNA Polymerase Assembly Factor Crl and Identification of Its Interaction Surface with Sigma S. J Bacteriol 196:3279-3288. doi: 10.1128/JB.01910-14

Barrientos A, Zambrano A, Tzagoloff A (2004) Mss51p and Cox14p jointly regulate mitochondrial Cox1p expression in Saccharomyces cerevisiae. The EMBO Journal 23:3472-3482. doi: 10.1038/sj.emboj.7600358

Bauerschmitt H, Mick DU, Deckers M, et al (2010) Ribosome-binding proteins Mdm38 and Mba1 display overlapping functions for regulation of mitochondrial translation. Mol Biol Cell 21:1937-1944. doi: 10.1091/mbc.E1002-0101

Becker L, Bannwarth M, Meisinger C, et al (2005) Preprotein translocase of the outer mitochondrial membrane: reconstituted Tom 40 forms a characteristic TOM pore. Journal of Molecular Biology 353:1011-1020. doi:

10.1016/j.jmb.2005.09.019

Becker T, Böttinger L, Pfanner N (2012) Mitochondrial protein import: from transport pathways to an integrated network. Trends Biochem Sci 37:85-91. doi: 10.1016/j.tibs.2011.11.004

Beckmann R, Herrmann JM (2015) Structural biology. Mitoribosome oddities. Science 348:288-289. doi: 10.1126/science.aab1054

Bohnert M, Rehling P, Guiard B, et al (2010) Cooperation of stop-transfer and conservative sorting mechanisms in mitochondrial protein transport. Current Biology 20:1227-1232. doi: 10.1016/j.cub.2010.05.058

Bonnefoy N, Fiumera HL, Dujardin G, Fox TD (2009) Roles of Oxa1-related innermembrane translocases in assembly of respiratory chain complexes. Biochimica et Biophysica Acta (BBA) - Molecular Cell Research 1793:60-70. doi: 10.1016/j.bbamcr.2008.05.004 
Borst P, Grivell LA (1971) Mitochondrial ribosomes. FEBS Lett 13:73-88.

Boumans H, Grivell LA, Berden JA (1998) The respiratory chain in yeast behaves as a single functional unit. Journal of Biological Chemistry 273:4872-4877.

Caldas TD, Yaagoubi El A, Richarme G (1998) Chaperone properties of bacterial elongation factor EF-Tu. Journal of Biological Chemistry 273:11478-11482.

Cermakian N, Ikeda TM, Cedergren R, Gray MW (1996) Sequences homologous to yeast mitochondrial and bacteriophage T3 and T7 RNA polymerases are widespread throughout the eukaryotic lineage. Nucleic Acids Res 24:648-654.

Chen L, Yang Y, Liu S, et al (2014) High Expression of Leucine Zipper-EF-Hand Containing Transmembrane Protein 1 Predicts Poor Prognosis in Head and Neck Squamous Cell Carcinoma. BioMed Research International 1-8. doi: $10.1155 / 2014 / 850316$

Chin JW, Martin AB, King DS, et al (2002) Addition of a photocrosslinking amino acid to the genetic code of Escherichiacoli. Proceedings of the National Academy of Sciences 99:11020-11024. doi: 10.1073/pnas.172226299

Daley DO, Clifton R, Whelan J (2002) Intracellular gene transfer: reduced hydrophobicity facilitates gene transfer for subunit 2 of cytochrome c oxidase. Proceedings of the National Academy of Sciences 99:10510-10515. doi: 10.1073/pnas. 122354399

Diffley JF, Stillman B (1988) Purification of a yeast protein that binds to origins of DNA replication and a transcriptional silencer. Proceedings of the National Academy of Sciences 85:2120-2124.

DiMauro S, Hirano M (2009) Pathogenesis and treatment of mitochondrial disorders. Adv Exp Med Biol 652:139-170. doi: 10.1007/978-90-481-28136_10

Dimmer KS, Fritz S, Fuchs F, et al (2002) Genetic basis of mitochondrial function and morphology in Saccharomyces cerevisiae. Mol Biol Cell 13:847-853. doi: 10.1091/mbc.01-12-0588

Dimmer KS, Navoni F, Casarin A, et al (2007) LETM1, deleted in Wolf Hirschhorn syndrome is required for normal mitochondrial morphology and cellular viability. Human Molecular Genetics 17:201-214. doi: 10.1093/hmg/ddm297

Doonan PJ, Chandramoorthy HC, Hoffman NE, et al (2014) LETM1-dependent mitochondrial Ca2+ flux modulates cellular bioenergetics and proliferation. FASEB J 28:4936-4949. doi: 10.1096/fj.14-256453

Dormán G, Prestwich GD (1994) Benzophenone photophores in biochemistry. Biochemistry 33:5661-5673.

Dudek J, Rehling P, van der Laan M (2013) Mitochondrial protein import: common principles and physiological networks. BBA - Molecular Cell Research 
1833:274-285. doi: 10.1016/j.bbamcr.2012.05.028

Ebright RH (2000) RNA polymerase: structural similarities between bacterial RNA polymerase and eukaryotic RNA polymerase II. Journal of Molecular Biology 304:687-698. doi: 10.1006/jmbi.2000.4309

Elstner M, Andreoli C, Klopstock T, et al (2009) The mitochondrial proteome database: MitoP2. Meth Enzymol 457:3-20. doi: 10.1016/S00766879(09)05001-0

Endele S, Fuhry M, Pak SJ, et al (1999) LETM1, a novel gene encoding a putative EFhand $\mathrm{Ca}(2+)$-binding protein, flanks the Wolf-Hirschhorn syndrome (WHS) critical region and is deleted in most WHS patients. Genomics 60:218-225. doi: 10.1006/geno.1999.5881

Farrell IS, Toroney R, Hazen JL, et al (2005) Photo-cross-linking interacting proteins with a genetically encoded benzophenone. Nat Methods 2:377-384. doi: 10.1038/nmeth0505-377

Fiori A, Pérez-Martínez X, Fox TD (2005) Overexpression of the COX2 translational activator, Pet111p, prevents translation of COX1 mRNA and cytochrome c oxidase assembly in mitochondria of Saccharomyces cerevisiae. Mol Microbiol 56:1689-1704. doi: 10.1111/j.1365-2958.2005.04658.x

Fox TD, Pérez-Martínez X, Butler CA, Shingú-Vázquez M (2009) Dual functions of Mss51 couple synthesis of Cox1 to assembly of cytochrome c oxidase in Saccharomyces cerevisiae mitochondria. Mol Biol Cell 20:4371-4380. doi: 10.1091/mbc.E09-06-0522

Frazier AE, Taylor RD, Mick DU, et al (2006) Mdm38 interacts with ribosomes and is a component of the mitochondrial protein export machinery. The Journal of Cell Biology 172:553-564. doi: 10.1083/jcb.200505060

Froschauer E, Nowikovsky K, Schweyen RJ (2005) Electroneutral K+/H+ exchange in mitochondrial membrane vesicles involves Yol027/Letm1 proteins. Biochimica et Biophysica Acta (BBA) - Biomembranes 1711:41-48. doi: 10.1016/j.bbamem.2005.02.018

Gardino AK, Smerdon SJ, Yaffe MB (2006) Structural determinants of 14-3-3 binding specificities and regulation of subcellular localization of 14-3-3-ligand complexes: a comparison of the X-ray crystal structures of all human 14-3-3 isoforms. Semin Cancer Biol 16:173-182. doi: 10.1016/j.semcancer.2006.03.007

Garofalo C, Trinko R, Kramer G, et al (2003) Purification and characterization of yeast mitochondrial initiation factor 2. Arch Biochem Biophys 413:243-252.

Gietz RD, Schiestl RH (2007) Frozen competent yeast cells that can be transformed with high efficiency using the LiAc/SS carrier DNA/PEG method. Nat Protoc 2:1-4. doi: $10.1038 /$ nprot.2007.17 
Gilbert C, Kristjuhan A, Winkler GS, Svejstrup JQ (2004) Elongator interactions with nascent mRNA revealed by RNA immunoprecipitation. Mol Cell 14:457464.

Glaser F, Pupko T, Paz I, et al (2003) ConSurf: identification of functional regions in proteins by surface-mapping of phylogenetic information. Bioinformatics 19:163-164.

Graack HR, Wittmann-Liebold B (1998) Mitochondrial ribosomal proteins (MRPs) of yeast. Biochem J 329 ( Pt 3):433-448.

Gray MW, Burger G, Lang BF (2001) The origin and early evolution of mitochondria. Genome Biology 2:reviews1018. doi: 10.1186/gb-2001-2-6reviews 1018

Greenberg JR (1979) Ultraviolet light-induced crosslinking of mRNA to proteins. Nucleic Acids Res 6:715-732.

Gruschke S, Gröne K, Heublein M, et al (2010) Proteins at the polypeptide tunnel exit of the yeast mitochondrial ribosome. J Biol Chem 285:19022-19028. doi: 10.1074/jbc.M110.113837

Gruschke S, Kehrein K, Römpler K, et al (2011) Cbp3-Cbp6 interacts with the yeast mitochondrial ribosomal tunnel exit and promotes cytochrome $b$ synthesis and assembly. The Journal of Cell Biology 193:1101-1114. doi: $10.1083 /$ jcb.201103132

Gruschke S, Ott M (2010) The polypeptide tunnel exit of the mitochondrial ribosome is tailored to meet the specific requirements of the organelle. Bioessays 32:1050-1057. doi: 10.1002/bies.201000081

Gruschke S, Römpler K, Hildenbeutel M, et al (2012) The Cbp3-Cbp6 complex coordinates cytochrome b synthesis with bc(1) complex assembly in yeast mitochondria. The Journal of Cell Biology 199:137-150. doi: 10.1083/jcb.201206040

Gualerzi CO, Pon CL (1990) Initiation of mRNA translation in prokaryotes. Biochemistry 29:5881-5889.

Guzman LM, Belin D, Carson MJ, Beckwith J (1995) Tight regulation, modulation, and high-level expression by vectors containing the arabinose PBAD promoter. J Bacteriol 177:4121-4130.

Hackenbrock CR, Chazotte B, Gupte SS (1986) The random collision model and a critical assessment of diffusion and collision in mitochondrial electron transport. J Bioenerg Biomembr 18:331-368.

Han D, Ye G, Liu T, et al (2010) Functional identification of a novel 14-3-3 epsilon splicing variant suggests dimerization is not necessary for 14-3-3 epsilon to inhibit UV-induced apoptosis. Biochem Biophys Res Commun 396:401-406. doi: 10.1016/j.bbrc.2010.04.104 
Hasegawa A, van der Bliek AM (2007) Inverse correlation between expression of the Wolfs Hirschhorn candidate gene Letm 1 and mitochondrial volume in C. elegans and in mammalian cells. Human Molecular Genetics 16:2061-2071. doi: $10.1093 / \mathrm{hmg} / \mathrm{ddm} 154$

Hashimi H, McDonald L, Stribrna E, Lukes J (2013) Trypanosome Letm1 Protein Is Essential for Mitochondrial Potassium Homeostasis. Journal of Biological Chemistry 288:26914-26925. doi: 10.1074/jbc.M113.495119

Hell K, Herrmann JM, Pratje E, et al (1998) Oxa1p, an essential component of the Ntail protein export machinery in mitochondria. Proceedings of the National Academy of Sciences 95:2250-2255.

Hell K, Neupert W, Stuart RA (2001) Oxa1p acts as a general membrane insertion machinery for proteins encoded by mitochondrial DNA. The EMBO Journal 20:1281-1288. doi: 10.1093/emboj/20.6.1281

Hieronymus H, Silver PA (2003) Genome-wide analysis of RNA-protein interactions illustrates specificity of the mRNA export machinery. Nature Genetics 33:155-161. doi: 10.1038/ng1080

Hildenbeutel M, Hegg EL, Stephan K, et al (2014) Assembly factors monitor sequential hemylation of cytochrome $b$ to regulate mitochondrial translation. The Journal of Cell Biology 205:511-524. doi: 10.1083/jcb.201401009

Hill JE, Myers AM, Koerner TJ, Tzagoloff A (1986) Yeast/E. coli shuttle vectors with multiple unique restriction sites. Yeast 2:163-167. doi: 10.1002/yea.320020304

Hirschhorn K, COOPER HL (1961) Chromosomal aberrations in human disease. A review of the status of cytogenetics in medicine. Am J Med 31:442-470.

Hoch FL (1992) Cardiolipins and biomembrane function. Biochimica et Biophysica Acta (BBA) - Bioenergetics 1113:71-133. doi: 10.1016/0304-4157(92)900359

Hoffmann A, Bukau B, Kramer G (2010) Structure and function of the molecular chaperone Trigger Factor. Biochim Biophys Acta 1803:650-661. doi: 10.1016/j.bbamcr.2010.01.017

Horan S, Bourges I, Taanman J-W, Meunier B (2005) Analysis of COX2 mutants reveals cytochrome oxidase subassemblies in yeast. Biochem J 390:703-708. doi: 10.1042/BJ20050598

Hwang S-K, Piao L, Lim H-T, et al (2010) Suppression of Lung Tumorigenesis by Leucine Zipper/EF Hand-Containing Transmembrane-1. PLoS ONE 5:e1253514. doi: 10.1371/journal.pone.0012535

Jia L, Dienhart M, Schramp M, et al (2003) Yeast Oxa1 interacts with mitochondrial ribosomes: the importance of the C-terminal region of Oxa1. The EMBO Journal 22:6438-6447. doi: 10.1093/emboj/cdg624 
Jia L, Kaur J, Stuart RA (2009) Mapping of the Saccharomyces cerevisiae Oxa1mitochondrial ribosome interface and identification of MrpL40, a ribosomal protein in close proximity to Oxa1 and critical for oxidative phosphorylation complex assembly. Eukaryotic Cell 8:1792-1802. doi: 10.1128/EC.00219-09

Jiang D, Zhao L, Clapham DE (2009) Genome-Wide RNAi Screen Identifies Letm1 as a Mitochondrial Ca2+/H+ Antiporter. Science 326:144-147. doi: 10.1126/science. 1175145

Jones CN, Miller C, Tenenbaum A, et al (2009) Antibiotic effects on mitochondrial translation and in patients with mitochondrial translational defects. Mitochondrion 9:429-437. doi: 10.1016/j.mito.2009.08.001

Kaziro Y (1978) The role of guanosine 5'-triphosphate in polypeptide chain elongation. Biochim Biophys Acta 505:95-127.

Kehrein K, Schilling R, Möller-Hergt BV, et al (2015) Organization of Mitochondrial Gene Expression in Two Distinct Ribosome-Containing Assemblies. CellReports 10:843-853. doi: 10.1016/j.celrep.2015.01.012

Keil M, Bareth B, Woellhaf MW, et al (2012) Oxa1-ribosome complexes coordinate the assembly of cytochrome $\mathrm{C}$ oxidase in mitochondria. J Biol Chem 287:34484-34493. doi: 10.1074/jbc.M112.382630

Kelly JL, Lehman IR (1986) Yeast mitochondrial RNA polymerase. Purification and properties of the catalytic subunit. Journal of Biological Chemistry 261:1034010347.

Kitakawa M, Isono K (1991) The mitochondrial ribosomes. Biochimie 73:813-825.

Koc EC, Spremulli LL (2002) Identification of mammalian mitochondrial translational initiation factor 3 and examination of its role in initiation complex formation with natural mRNAs. Journal of Biological Chemistry 277:3554135549. doi: 10.1074/jbc.M202498200

Komiya T, Rospert S, Schatz G, Mihara K (1997) Binding of mitochondrial precursor proteins to the cytoplasmic domains of the import receptors Tom70 and Tom 20 is determined by cytoplasmic chaperones. The EMBO Journal 16:4267-4275.

Komuro Y, Miyashita N, Mori T, et al (2013) Energetics of the presequence-binding poses in mitochondrial protein import through Tom20. J Phys Chem B 117:2864-2871. doi: 10.1021/jp400113e

Krause K, Lopes de Souza R, Roberts DGW, Dieckmann CL (2004) The mitochondrial message-specific mRNA protectors Cbp1 and Pet309 are associated in a high-molecular weight complex. Mol Biol Cell 15:2674-2683. doi: 10.1091/mbc.E04-02-0126

Krause-Buchholz U, Schöbel K, Lauffer S, Rödel G (2005) Saccharomyces cerevisiae translational activator Cbs1p is associated with translationally active 
mitochondrial ribosomes. Biol Chem 386:407-415. doi: 10.1515/BC.2005.049

Krüger V, Deckers M, Hildenbeutel M, et al (2012) The mitochondrial oxidase assembly protein 1 (Oxa1) insertase forms a membrane pore in lipid bilayers. J Biol Chem 287:33314-33326. doi: 10.1074/jbc.M112.387563

Kuo D, Nie M, Courey AJ (2014) SUMO as a solubility tag and in vivo cleavage of SUMO fusion proteins with Ulp1. Methods Mol Biol 1177:71-80. doi: 10.1007/978-1-4939-1034-2_6

Kuum M, Veksler V, Liiv J, et al (2012) Endoplasmic reticulum potassium-hydrogen exchanger and small conductance calcium-activated potassium channel activities are essential for ER calcium uptake in neurons and cardiomyocytes. Journal of Cell Science 125:625-633. doi: 10.1242/jcs.090126

Kuzmenko A, Atkinson GC, Levitskii S, et al (2014) Mitochondrial translation initiation machinery: Conservation and\&nbsp;diversification. Biochimie 100:132-140. doi: 10.1016/j.biochi.2013.07.024

Leitner A, Walzthoeni T, Kahraman A, et al (2010) Probing native protein structures by chemical cross-linking, mass spectrometry, and bioinformatics. Mol Cell Proteomics 9:1634-1649. doi: 10.1074/mcp.R000001-MCP201

Leon SA, Mahler HR (1968) Isolation and properties of mitochondrial RNA from yeast. Arch Biochem Biophys 126:305-319.

Lill R (2009) Function and biogenesis of iron-sulphur proteins. Nature 460:831838. doi: $10.1038 /$ nature08301

Lupo D, Vollmer C, Deckers M, et al (2011) Mdm38 is a 14-3-3-like receptor and associates with the protein synthesis machinery at the inner mitochondrial membrane. Traffic 12:1457-1466. doi: 10.1111/j.1600-0854.2011.01239.x

Manthey GM, McEwen JE (1995) The product of the nuclear gene PET309 is required for translation of mature mRNA and stability or production of introncontaining RNAs derived from the mitochondrial COX1 locus of Saccharomyces cerevisiae. The EMBO Journal 14:4031-4043.

Marshall RA, Aitken CE, Dorywalska M, Puglisi JD (2008) Translation at the singlemolecule level. Annu Rev Biochem 77:177-203. doi:

10.1146/annurev.biochem.77.070606.101431

Martinez-Hackert E, Hendrickson WA (2009) Promiscuous substrate recognition in folding and assembly activities of the trigger factor chaperone. Cell 138:923934. doi: 10.1016/j.cell.2009.07.044

Martinou J-C, Youle RJ (2011) Mitochondria in apoptosis: Bcl-2 family members and mitochondrial dynamics. Cell 21:92-101. doi:

10.1016/j.devcel.2011.06.017

Merz F, Boehringer D, Schaffitzel C, et al (2008) Molecular mechanism and 
structure of Trigger Factor bound to the translating ribosome. The EMBO Journal 27:1622-1632. doi: 10.1038/emboj.2008.89

Mick DU, Vukotic M, Piechura H, et al (2010) Coa3 and Cox14 are essential for negative feedback regulation of COX1 translation in mitochondria. The Journal of Cell Biology 191:141-154. doi: 10.1083/jcb.201007026

Mileykovskaya E, Dowhan W (2009) Cardiolipin membrane domains in prokaryotes and eukaryotes. Biochim Biophys Acta 1788:2084-2091. doi: 10.1016/j.bbamem.2009.04.003

Miller JH, Albertini AM (1983) Effects of surrounding sequence on the suppression of nonsense codons. Journal of Molecular Biology 164:59-71.

Mills DA, Hosler JP, Ferguson-Miller S (2006) Energy transduction: proton transfer through the respiratory complexes. Annu Rev Biochem 75:165-187. doi: 10.1146/annurev.biochem.75.062003.101730

Milon P, Konevega AL, Peske F, et al (2007) Transient kinetics, fluorescence, and FRET in studies of initiation of translation in bacteria. Meth Enzymol 430:1-30. doi: 10.1016/S0076-6879(07)30001-3

Miroux B, Walker JE (1996) Over-production of proteins in Escherichia coli: mutant hosts that allow synthesis of some membrane proteins and globular proteins at high levels. Journal of Molecular Biology 260:289-298. doi: 10.1006/jmbi.1996.0399

Moriya H, Kasai H, Isono K (1995) Cloning and characterization of the hrpA gene in the terC region of Escherichia coli that is highly similar to the DEAH family RNA helicase genes of Saccharomyces cerevisiae. Nucleic Acids Res 23:595598.

Nagata S, Tsunetsugu-Yokota Y, Naito A, Kaziro Y (1983) Molecular cloning and sequence determination of the nuclear gene coding for mitochondrial elongation factor Tu of Saccharomyces cerevisiae. Proceedings of the National Academy of Sciences 80:6192-6196.

Naithani S, Saracco SA, Butler CA, Fox TD (2003) Interactions among COX1, COX2, and COX3 mRNA-specific translational activator proteins on the inner surface of the mitochondrial inner membrane of Saccharomyces cerevisiae. Mol Biol Cell 14:324-333. doi: 10.1091/mbc.E02-08-0490

Neuhoff V, Stamm R, Eibl H (1985) Clear background and highly sensitive protein staining with Coomassie Blue dyes in polyacrylamide gels:. Electrophoresis 427-488.

Nowikovsky K, Froschauer EM, Zsurka G, et al (2004) The LETM1/YOL027 gene family encodes a factor of the mitochondrial $\mathrm{K}+$ homeostasis with a potential role in the Wolf-Hirschhorn syndrome. Journal of Biological Chemistry 279:30307-30315. doi: 10.1074/jbc.M403607200 
Nowikovsky K, Reipert S, Devenish RJ, Schweyen RJ (2007) Mdm38 protein depletion causes loss of mitochondrial $\mathrm{K}+\mathrm{H}+$ exchange activity, osmotic swelling and mitophagy. Cell Death Differ 14:1647-1656. doi: 10.1038/sj.cdd.4402167

Ott M, Herrmann JM (2010) Co-translational membrane insertion of mitochondrially encoded proteins. Biochim Biophys Acta 1803:767-775. doi: 10.1016/j.bbamcr.2009.11.010

Ott M, Prestele M, Bauerschmitt H, et al (2006) Mba1, a membrane-associated ribosome receptor in mitochondria. The EMBO Journal 25:1603-1610. doi: 10.1038/sj.emboj.7601070

Ottmann C, Yasmin L, Weyand M, et al (2007) Phosphorylation-independent interaction between 14-3-3 and exoenzyme S: from structure to pathogenesis. The EMBO Journal 26:902-913. doi: 10.1038/sj.emboj.7601530

Park E, Rapoport TA (2012) Mechanisms of Sec61/SecY-mediated protein translocation across membranes. Annu Rev Biophys 41:21-40. doi: 10.1146/annurev-biophys-050511-102312

PerezMartinez X, Broadley SA, Fox TD (2003) Mss51p promotes mitochondrial Cox1p synthesis and interacts with newly synthesized Cox1p. The EMBO Journal 22:5951-5961. doi: 10.1093/emboj/cdg566

Pfeffer S, Woellhaf MW, Herrmann JM, Förster F (2015) Organization of the mitochondrial translation machinery studied in situ by cryoelectron tomography. Nat Commun 6:6019. doi: 10.1038/ncomms7019

Piao L, Li Y, Kim SJ, et al (2009) Association of LETM1 and MRPL36 contributes to the regulation of mitochondrial ATP production and necrotic cell death. Cancer Res 69:3397-3404. doi: 10.1158/0008-5472.CAN-08-3235

Preuss M, Leonhard K, Hell K, et al (2001) Mba1, a novel component of the mitochondrial protein export machinery of the yeast Saccharomyces cerevisiae. The Journal of Cell Biology 153:1085-1096.

Reif S, Randelj O, Domanska GY, et al (2005) Conserved mechanism of Oxa1 insertion into the mitochondrial inner membrane. Journal of Molecular Biology 354:520-528. doi: 10.1016/j.jmb.2005.09.054

Remedios dos CG, Moens PD (1995) Fluorescence resonance energy transfer spectroscopy is a reliable "ruler" for measuring structural changes in proteins. Dispelling the problem of the unknown orientation factor. J Struct Biol 115:175-185. doi: 10.1006/jsbi.1995.1042

Repetto B, Tzagoloff A (1990) Structure and regulation of KGD2, the structural gene for yeast dihydrolipoyl transsuccinylase. Mol Cell Biol 10:4221-4232.

Rhee H-W, Zou P, Udeshi ND, et al (2013) Proteomic mapping of mitochondria in living cells via spatially restricted enzymatic tagging. Science 339:1328-1331. 
doi: $10.1126 /$ science.1230593

Rimessia A, Giorgia C, Pintona P, Rizzutoa R (2008) The versatility of mitochondrial calcium signals: from stimulation of cell metabolism to induction of cell death. Biochimica et Biophysica Acta (BBA) - Bioenergetics 1777:808-816. doi: 10.1016/j.bbabio.2008.05.449

Rittinger K, Budman J, Xu J, et al (1999) Structural analysis of 14-3-3 phosphopeptide complexes identifies a dual role for the nuclear export signal of 14-3-3 in ligand binding. Mol Cell 4:153-166.

Rodeheffer MS, Boone BE, Bryan AC, Shadel GS (2001) Nam1p, a protein involved in RNA processing and translation, is coupled to transcription through an interaction with yeast mitochondrial RNA polymerase. Journal of Biological Chemistry 276:8616-8622. doi: 10.1074/jbc.M009901200

Roux KJ, Kim DI, Raida M, Burke B (2012) A promiscuous biotin ligase fusion protein identifies proximal and interacting proteins in mammalian cells. The Journal of Cell Biology 196:801-810. doi: 10.1083/jcb.201112098

Rödel G (1986) Two yeast nuclear genes, CBS1 and CBS2, are required for translation of mitochondrial transcripts bearing the 5 '-untranslated COB leader. Curr Genet 11:41-45. doi: 10.1007/BF00389424

Ryu Y, Schultz PG (2006) Efficient incorporation of unnatural amino acids into proteins in Escherichia coli. Nat Methods 3:263-265. doi: 10.1038/nmeth864

Sahaf B, Heydari K, Herzenberg LA, Herzenberg LA (2003) Lymphocyte surface thiol levels. Proceedings of the National Academy of Sciences 100:4001-4005. doi: $10.1073 /$ pnas. 2628032100

Salman-Dilgimen A, Hardy P-O, Radolf JD, et al (2013) HrpA, an RNA helicase involved in RNA processing, is required for mouse infectivity and tick transmission of the Lyme disease spirochete. PLoS Pathog 9:e1003841. doi: 10.1371/journal.ppat.1003841

Sambrook J, Maniatis EF, Fritsch T (1989) Molecular cloning: a laboratory manual. Cold Spring Harbor Laboratory Press, Cold Spring Harbor

Sansom MS, Weinstein H (2000) Hinges, swivels and switches: the role of prolines in signalling via transmembrane alpha-helices. Trends Pharmacol Sci 21:445451.

Saracco SA, Fox TD (2002) Cox18p is required for export of the mitochondrially encoded Saccharomyces cerevisiae Cox2p C-tail and interacts with Pnt1p and Mss2p in the inner membrane. Mol Biol Cell 13:1122-1131. doi: 10.1091/mbc.01-12-0580

Schägger H (2001) Respiratory chain supercomplexes. IUBMB Life 52:119-128. doi: $10.1080 / 15216540152845911$ 
Schägger H, Jagow von G (1991) Blue native electrophoresis for isolation of membrane protein complexes in enzymatically active form. Anal Biochem 199:223-231.

Schimper AFW (1883) Über die Entwicklung der Chlorophyllkörner und Farbkörper. Botanische Zeitung 1-17.

Schlickum S, Moghekar A, Simpson JC, et al (2004) LETM1, a gene deleted in WolfHirschhorn syndrome, encodes an evolutionarily conserved mitochondrial protein. Genomics 83:254-261. doi: 10.1016/j.ygeno.2003.08.013

Selmer M, Al-Karadaghi S, Hirokawa G, et al (1999) Crystal structure of Thermotoga maritima ribosome recycling factor: a tRNA mimic. Science 286:2349-2352.

Sicheritz-Pontén T, Kurland CG, Andersson SG (1998) A phylogenetic analysis of the cytochrome $b$ and cytochrome c oxidase I genes supports an origin of mitochondria from within the Rickettsiaceae. Biochim Biophys Acta 1365:545551.

Sikorski RS, Hieter P (1989) A system of shuttle vectors and yeast host strains designed for efficient manipulation of DNA in Saccharomyces cerevisiae. Genetics 122:19-27.

Sluchanko NN, Sudnitsyna MV, Chernik IS, et al (2011) Phosphomimicking mutations of human 14-3-3 3 affect its interaction with tau protein and small heat shock protein HspB6. Arch Biochem Biophys 506:24-34. doi: 10.1016/j.abb.2010.11.003

Smits P, Smeitink JAM, van den Heuvel LP, et al (2007) Reconstructing the evolution of the mitochondrial ribosomal proteome. Nucleic Acids Res 35:4686-4703. doi: 10.1093/nar/gkm441

Soto IC, Fontanesi F, Liu J, Barrientos A (2012) Biogenesis and assembly of eukaryotic cytochrome c oxidase catalytic core. Biochimica et Biophysica Acta (BBA) - Bioenergetics 1817:883-897. doi: 10.1016/j.bbabio.2011.09.005

South ST, Whitby H, Battaglia A, et al (2008) Comprehensive analysis of WolfHirschhorn syndrome using array CGH indicates a high prevalence of translocations. Eur J Hum Genet 16:45-52. doi: 10.1038/sj.ejhg.5201915

Stryer L (1978) Fluorescence energy transfer as a spectroscopic ruler. Annu Rev Biochem 47:819-846. doi: 10.1146/annurev.bi.47.070178.004131

Stuart RA, Rehling P (2008) Mitochondrial biogenesis: is an old dog still teaching us new tricks? Meeting on the Assembly of the Mitochondrial Respiratory Chain. In: EMBO Rep. pp 33-38

Tamai S, Iida H, Yokota S, et al (2008) Characterization of the mitochondrial protein LETM1, which maintains the mitochondrial tubular shapes and interacts with the AAA-ATPase BCS1L. Journal of Cell Science 121:2588-2600. 
doi: $10.1242 /$ jcs.026625

Tavares-Carreón F, Camacho-Villasana Y, Zamudio-Ochoa A, et al (2008) The pentatricopeptide repeats present in Pet309 are necessary for translation but not for stability of the mitochondrial COX1 mRNA in yeast. Journal of Biological Chemistry 283:1472-1479. doi: 10.1074/jbc.M708437200

Teyssier E, Hirokawa G, Tretiakova A, et al (2003) Temperature-sensitive mutation in yeast mitochondrial ribosome recycling factor (RRF). Nucleic Acids Res $31: 4218-4226$.

Towpik J, Chacinska A, Ciesla M, et al (2004) Mutations in the yeast mrf1 gene encoding mitochondrial release factor inhibit translation on mitochondrial ribosomes. Journal of Biological Chemistry 279:14096-14103. doi: 10.1074/jbc.M312856200

Tsai M-F, Jiang D, Zhao L, et al (2014) Functional reconstitution of the mitochondrial $\mathrm{Ca} 2+/ \mathrm{H}+$ antiporter Letm 1 . The Journal of General Physiology 143:67-73. doi: 10.1085/jgp.201311096

Tzschoppe K, Kohlwein SD, Rödel G (2000) Yeast translational activator Cbs2p: mitochondrial targeting and effect of overexpression. Biol Chem 381:11751183. doi: 10.1515/BC.2000.145

Vambutas A, Ackerman SH, Tzagoloff A (1991) Mitochondrial translationalinitiation and elongation factors in Saccharomyces cerevisiae. Eur J Biochem 201:643-652.

van der Laan M, Hutu DP, Rehling P (2010) On the mechanism of preprotein import by the mitochondrial presequence translocase. Biochim Biophys Acta 1803:732-739. doi: 10.1016/j.bbamcr.2010.01.013

Vestweber D, Brunner J, Baker A, Schatz G (1989) A 42K outer-membrane protein is a component of the yeast mitochondrial protein import site. Nature 341:205-209. doi: 10.1038/341205a0

Vögtle F-N, Wortelkamp S, Zahedi RP, et al (2009) Global analysis of the mitochondrial N-proteome identifies a processing peptidase critical for protein stability. Cell 139:428-439. doi: 10.1016/j.cell.2009.07.045

Waldeck-Weiermair M, Jean-Quartier C, Rost R, et al (2011) Leucine zipper EF hand-containing transmembrane protein 1 (Letm1) and uncoupling proteins 2 and 3 (UCP2/3) contribute to two distinct mitochondrial Ca2+ uptake pathways. J Biol Chem 286:28444-28455. doi: 10.1074/jbc.M111.244517

Wallis MG, Groudinsky O, Slonimski PP, Dujardin G (1994) The NAM1 protein (NAM1p), which is selectively required for cox1, cytb and atp6 transcript processing/stabilisation, is located in the yeast mitochondrial matrix. Eur J Biochem 222:27-32.

Wiesenberger G, Costanzo MC, Fox TD (1995) Analysis of the Saccharomyces 
cerevisiae mitochondrial COX3 mRNA 5' untranslated leader: translational activation and mRNA processing. Mol Cell Biol 15:3291-3300.

Wright TJ, Ricke DO, Denison K, et al (1997) A transcript map of the newly defined $165 \mathrm{~kb}$ Wolf-Hirschhorn syndrome critical region. Human Molecular Genetics 6:317-324.

Yaffe MB, Rittinger K, Volinia S, et al (1997) The structural basis for 14-33:phosphopeptide binding specificity. Cell 91:961-971.

Zambrano A, Fontanesi F, Solans A, et al (2007) Aberrant translation of cytochrome c oxidase subunit 1 mRNA species in the absence of Mss51p in the yeast Saccharomyces cerevisiae. Mol Biol Cell 18:523-535. doi: 10.1091/mbc.E06-09-0803

Zhang B, Carrie C, Ivanova A, et al (2012) LETM Proteins Play a Role in the Accumulation of Mitochondrially Encoded Proteins in Arabidopsis thaliana and AtLETM2 Displays Parent of Origin Effects. Journal of Biological Chemistry 287:41757-41773. doi: 10.1074/jbc.M112.383836

Zollino M, Lecce R, Fischetto R, et al (2003) Mapping the Wolf-Hirschhorn syndrome phenotype outside the currently accepted WHS critical region and defining a new critical region, WHSCR-2. Am J Hum Genet 72:590-597. doi: $10.1086 / 367925$

Zollino M, Wright TJ, Di Stefano C, et al (1999) "Tandem" duplication of 4p16.1p16.3 chromosome region associated with 4p16.3pter molecular deletion resulting in Wolf-Hirschhorn syndrome phenotype. Am J Med Genet 82:371-375. doi: 10.1002/(SICI)1096-8628(19990219)82:5<371::AIDAJMG3>3.0.CO;2-J

Zotova L, Aleschko M, Sponder G, et al (2010) Novel components of an active mitochondrial $\mathrm{K}(+) / \mathrm{H}(+)$ exchange. J Biol Chem 285:14399-14414. doi: 10.1074/jbc.M109.059956 